\title{
The cosmological constant and the energy of gravitational radiation*
}

\author{
Piotr T. Chruściel ${ }^{\dagger}$ and Lukas Ifsits ${ }^{\ddagger}$ \\ Erwin Schrödinger Institute and Faculty of Physics \\ University of Vienna
}

March 6, 2018

\begin{abstract}
We propose a definition of mass for characteristic hypersurfaces in asymptotically vacuum space-times with non-vanishing cosmological constant $\Lambda \in \mathbb{R}^{*}$, generalising the definition of Trautman and Bondi for $\Lambda=0$. We show that our definition reduces to some standard definitions in several situations. We establish a balance formula linking the characteristic mass and a suitably defined renormalised volume of the null hypersurface, generalising the positivity identity of one of us (PTC) and Paetz proved when $\Lambda=0$.
\end{abstract}

PACS: 04.20.Cv, 04.20.Ex, 04.20.Ha

\section{Contents}

$\begin{array}{ll}\text { Contents } & 1\end{array}$

1 Introduction 3

2 Boundary conditions $\quad 5$

2.1 Bondi coordinates . . . . . . . . . . . . . . 5

2.2 Fefferman-Graham expansions . . . . . . . . . . . 8

2.3 The next term and the geometry of $\mathscr{N} \ldots \ldots . \ldots . \ldots 9$

3 Characteristic hypersurfaces $r$

3.1 Wave-map gauge . . . . . . . . . . . . . . . . 13

3.2 Characteristic surfaces, adapted null coordinates and assumptions on the metric . . . . . . . . . . . . . . 13

*Preprint UWThPh-2016-4. Supported in part by the Austrian Science Fund (FWF): P 23719-N16. PTC is grateful to the Center for Mathematical Sciences and Applications, Harvard, and to Monash University for hospitality and support during part of work on this paper.

${ }^{\dagger}$ Email piotr.chrusciel@univie.ac.at; URL http://homepage.univie.ac.at/piotr. chrusciel

${ }^{\ddagger}$ Email lukas.ifsits@univie.ac.at 
4 Asymptotic solutions of the characteristic wave-map gauge constraints, $\Lambda \neq 0 \quad 16$

4.1 Matter fields . . . . . . . . . . . . . . 16

4.2 Solving equation $(3.11) \ldots \ldots \ldots \ldots$

4.3 Expansion of $\nu_{\mathrm{Bo}}^{0} \ldots \ldots \ldots \ldots \ldots$

4.4 Expansion of $\xi_{A}^{\mathrm{Bo}} \ldots \ldots \ldots \ldots \ldots \ldots \ldots$

4.5 Expansion of $\nu_{\mathrm{Bo}}^{A} \ldots \ldots \ldots \ldots \ldots \ldots$

4.6 Expansion of $\zeta^{\mathrm{Bo}} \ldots \ldots \ldots \ldots \ldots$

4.7 Analyzing $(3.16) \ldots \ldots \ldots \ldots . \ldots \ldots . \ldots \ldots$

4.8 No-logs . . . . . . . . . . . . . . . . . 22

5 Characteristic mass $\quad 22$

5.1 The Trautman-Bondi mass . . . . . . . . . . . . . . . . 22

5.2 The characteristic mass in Bondi-type coordinates . . . . . . . . 24

5.3 The characteristic mass in terms of characteristic data . . . . . 25

5.4 The characteristic mass and the renormalized volume . . . . . . 28

6 Coordinate mass $\quad 35$

6.1 Birmingham metrics . . . . . . . . . . . . . . 35

6.2 Horowitz-Myers-type metrics . . . . . . . . . . . 36

6.2 .1 The metric . . . . . . . . . . . . 36

$6.2 .2 \quad \beta=0, n=3 \ldots \ldots \ldots \ldots \ldots \ldots$

$6.2 .3 \quad \beta= \pm 1, n=3 \ldots \ldots \ldots \ldots \ldots$

6.2.4 Negative coordinate mass . . . . . . . . . . 38

$\begin{array}{llr}7 & \text { Examples } & \mathbf{4 0}\end{array}$

7.1 Birmingham metrics . . . . . . . . . . . . . . . 40

7.1.1 Mass and volume . . . . . . . . . . . . . 41

7.1.2 The balance equation for Birmingham metrics . . . . . . 41

7.2 Horowitz-Myers type metrics . . . . . . . . . . . . . . . 42

7.2.1 Bondi coordinates, characteristic mass . . . . . . . . 43

7.2.2 Renormalized volume . . . . . . . . . . . . . 44

8 Hamiltonian mass, $\Lambda<0 \quad 45$

8.1 Asymptotically Birmingham metrics . . . . . . . . . . . 46

8.2 Asymptotically HM-type metrics . . . . . . . . . . . . . 51

8.3 Fefferman-Graham asymptotics with an ultrastatic conformal infinity . . . . . . . . . . . . . . . . 5 53

$\begin{array}{lll}9 & \text { Conclusions } & 57\end{array}$

A Null geometry of Horowitz-Myers metrics and the balance equation

References 


\section{Introduction}

While the notion of total mass of general relativistic gravitating systems with $\Lambda \leq 0$ is well understood by now (cf., eg., [8] and references therein), the notion of energy in the radiating regime in the presence of a positive cosmological constant appears to be largely unexplored (see, however, $[2,3,32]$ ). The object of this work is to contribute to filling this gap.

In this paper we address the question of properties of total mass and energy for radiating systems when $\Lambda \neq 0$. This will be done in the spirit of the pioneering work by Bondi et al. [5,31], by analysing the asymptotic behavior of the gravitational field on characteristic hypersurfaces extending to asymptotic regions. Many formal aspects of the problem turn out to be independent of the sign of the cosmological constant, and while we are mainly interested in the case $\Lambda>0$, we allow $\Lambda<0$ wherever relevant as several results below apply regardless of the sign of $\Lambda$. The case $\Lambda<0$ becomes a useful test-bed for the quantities involved in those aspects thereof which are well understood. It should, however, be emphasised that many of our results, such as e.g. the balance equation (5.56), are new both for $\Lambda<0$ and $\Lambda>0$.

It should be kept in mind that an elegant approach to the definition of energy has been proposed in [1] for field configurations that asymptotically approach a preferred background with Killing vectors. This provides a widely accepted definition of asymptotic charges in the case where $\Lambda \leq 0$. The approach of [1] does not work for non-trivial radiating fields with $\Lambda>0$, where no natural asymptotic background is known to exist. In retrospect, our work below can be used to provide such a background, namely the metric obtained by keeping only the leading order terms of $g$ in Bondi coordinates, but the decay rates of the metric to this background do not appear to be compatible with what is needed in the Abbott-Deser prescription.

The first issue that one needs to address is that of boundary conditions satisfied by the fields. A popular approach is to assume smooth conformal compactifiability of the space-time, and we develop a framework which covers such fields. We start by deriving in Section 2 below the restrictions on the free characteristic initial data that follow from existence of smooth conformal compactification. In particular, in Proposition 2.1 below we generalise to all $\Lambda \in \mathbb{R}$ a result established in [34] for $\Lambda=0$, that existence of a smooth conformal compactification guarantees existence of Bondi coordinates in which the metric coefficients have full asymptotic expansions in terms of inverse powers of the Bondi coordinate $r_{\mathrm{Bo}}$. In Section 3 we review those aspects of the characteristic Cauchy problem which are relevant for the issues at hand. In Section 4 we derive the asymptotic expansions of the metric along the characteristic surfaces. Our analysis is similar in spirit to that of $[18,28]$; however, here the asymptotic expansions have to be carried-out to higher orders because of new $\Lambda$-dependent nonlinear couplings between some asymptotic expansion coefficients. We also allow matter sources, while vacuum was assumed in [28]. In particular in Section 4.8 we derive the conditions (4.43) on the free initial data in Bondi-type coordinates which are necessary for absence of log terms in the metric.

The asymptotic expansions of Section 4 lead naturally, in Section 5, to the 
definition of a quantity analogous to the Trautman-Bondi mass. We derive there a key integral identity expressing this mass in terms of the free initial data and the renormalised volume of the characteristic surface, Equation (5.56). This is one of the main results of this work.

When $\Lambda=0$, our mass identity (5.56) reduces to the one derived in [17] (compare [33]), giving then an elementary proof of positivity of the TrautmanBondi energy for space-times containing globally smooth light-cones extending smoothly to $\mathscr{I}^{+}$. (As is well known the global structure of such space-times depends crucially upon the sign of $\Lambda$, see Figure 1.1.) In addition to the renor-

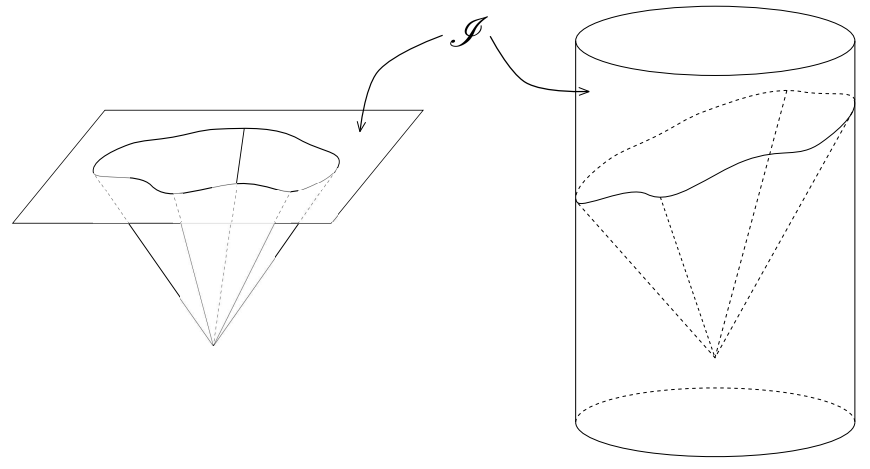

Figure 1.1: Globally smooth light-cones in space-times with a smooth conformal completion at a conformal boundary $\mathscr{I}$ at timelike infinity $(\Lambda>0$, left $)$ or spacelike infinity $(\Lambda<0$, right).

malised volume, boundary terms, and volume integrals involving the free data, the new formula, for asymptotically empty metrics with $\Lambda \neq 0$, involves several terms depending upon coefficients determined by the asymptotic behaviour of the fields multiplied by $\Lambda$. One can think of this equation as a balance formula relating the mass with the remaining quantities at hand.

To get some insight into the formula, in the remaining sections we turn our attention to the case $\Lambda<0$, where energy is much better understood. We review the notion of coordinate mass in Section 6 . We calculate the various quantities appearing in the mass identity (5.56) for the Birmingham metrics and the Horowitz-Myers (HM) metrics in Section 7. In Section 8 we derive simple formulae for the Hamiltonian mass for asymptotically Birmingham and asymptotically Horowitz-Myers metrics, in all space-times dimensions $n+1 \geq$ $4,{ }^{1}$ and for smoothly conformally compactifiable four-dimensional space-times with an ultrastatic conformal boundary. These formulae are used to show that the Hamiltonian mass coincides with the characteristic mass for a family of null hypersurfaces. In Appendix A we examine separately various contributions to our "energy balance" equation for Horowitz-Myers metrics.

Unless explicitly indicated otherwise, we assume throughout that $\Lambda \neq 0$.

\footnotetext{
${ }^{1}$ This extends the analysis in [10] and references therein to higher dimensions with the above boundary conditions.
} 


\section{Boundary conditions}

Consider an $(n+1)$-dimensional smoothly conformally compactifiable spacetime $(\mathscr{M}, g), n \geq 2$, solution of the vacuum Einstein equations with cosmological constant $\Lambda \neq 0$. By definition, there exists a manifold $(\widetilde{\mathscr{M}}, \widetilde{g})$ with boundary $\partial \widetilde{\mathscr{M}}$ and a defining function $\Omega$ for $\partial \widetilde{\mathscr{M}}$ such that

$$
g=\Omega^{-2} \widetilde{g}, \quad \partial \widetilde{\mathscr{M}}=\{\Omega=0\}, \quad d \Omega(p) \neq 0 \text { for } p \in \partial \widetilde{\mathscr{M}} .
$$

Again by definition, both $\Omega$ and $\widetilde{g}$ are smooth.

\subsection{Bondi coordinates}

In the asymptotically flat case, in spacetime dimension 4 and assuming $\Lambda=$ 0 , Bondi et al. have introduced a set of coordinates convenient for analysing gravitational radiation [5]. We will refer to them as Bondi coordinates. In these coordinates the metric takes the form

$$
\bar{g}=\bar{g}_{00} d u^{2}-2 e^{2 \omega} d r d u-2 r^{2} U_{A} d x^{A} d u+r^{2} \underbrace{h_{A B} d x^{A} d x^{B}}_{=: h},
$$

where the determinant of $h_{A B}$ is $r$-independent. ${ }^{2}$ (One further requires the fields $g_{00}, U_{A}, \omega$ and $h_{A B}$ to fulfill appropriate asymptotic conditions.) When using Bondi coordinates, we will decorate all fields and coordinates with a symbol "Bo". Existence of such coordinates in asymptotically vacuum space-times with $\Lambda=0$ and admitting smooth conformal completions has been established in [34], and in [14] for polyhomogeneous $\mathscr{I}$ 's.

We wish to prove existence of such coordinates, and to derive the asymptotic behaviour of smoothly compactifiable metrics in those coordinates in a neighborhood of the conformal boundary, with $\Lambda \in \mathbb{R}^{*}$. It turns out that, similarly to the $\Lambda=0$ case (cf., e.g., [14, Section 4], compare [28]), smoothness imposes restrictions on some lower-order coefficients in the asymptotic expansion of the free data on the null hypersurfaces meeting the conformal boundary smoothly and transversally.

Let, thus, $\Lambda \in \mathbb{R}$, and let $y^{0}: \partial \widetilde{\mathscr{M}} \rightarrow \mathbb{R}$ be a smooth function defined on an open subset of the conformal boundary $\partial \widetilde{\mathscr{M}}$, with $d y^{0}$ without zeros, such that the level sets

$$
S_{c}:=\left\{y^{0}=c\right\} \subset \partial \widetilde{\mathscr{M}}
$$

of $y^{0}$ form a smooth foliation by spacelike submanifolds. Passing to a subset of $\partial \widetilde{\mathscr{M}}$ if necessary, we can assume that $y^{0}$ is defined throughout $\partial \widetilde{\mathscr{M}}$.

So far $y^{0}$ has only been defined on the conformal boundary. Note that the gradient of $y^{0}$ within the boundary will be necessary timelike when $\partial \widetilde{\mathscr{M}}$ is timelike, and spacelike when $\partial \widetilde{\mathscr{M}}$ is spacelike. Nevertheless, we will extend $y^{0}$ to a function in space-time so that $\nabla y^{0}$ is null regardless of the causal character of the conformal boundary.

\footnotetext{
${ }^{2}$ We have used the symbol $\omega=\omega\left(u, r, x^{A}\right)$ for a function which is usually denoted by $\beta$ in the literature to avoid a conflict of notation with a constant $\beta$ elsewhere in the paper.
} 
Now, at every $p \in S_{c}$ there exists a unique vector $\stackrel{\circ}{X}_{p}$ which is null, future directed, outwards pointing, orthogonal to $T_{p} S_{c}$, and normalised to unit length with respect to some smooth auxiliary Riemannian metric. This defines a smooth vector field $\dot{X}$ on $\partial \widetilde{\mathscr{M}}$. We choose time-orientation so that $-X_{p}$ points towards the physical space-time.

Let $\gamma_{p}$ denote a maximally extended null geodesic with initial tangent $-\stackrel{\circ}{X}_{p}$ at $p$. Standard transversality and injectivity-radius arguments show that there exists a neighborhood $\mathscr{O}$ of $\widetilde{\mathscr{M}}$ such that for every $c \in \mathbb{R}$ the union of the (images of the) null geodesics

$$
\mathscr{N}_{c}:=\cup_{p \in S_{c}} \gamma_{p}
$$

forms a smooth null hypersurface, with $\cup_{c} S_{c}$ foliating $\mathscr{O}$.

To obtain Bondi-type coordinates we proceed now as follows:

1. Let $x^{A}$ denote local coordinates on $S_{c}$, in $3+1$ space-time dimensions we choose the conformal representative $\tilde{g}_{A B}$ of the metric induced on $S_{c}$ by $\widetilde{g}$ to take a canonical form. For example, if $S_{0}$ is diffeomorphic to a two-dimensional sphere, we choose $\stackrel{\tilde{g}}{A B}_{A B}$ to be the canonical metric $s_{A B}$ on $S^{2}$. In higher dimension one might wish to require instead that the volume element $\sqrt{\operatorname{det} \stackrel{\check{g}}{A B}_{A B}}$ takes a convenient form, depending upon the geometry of the conformal boundary.

2. We extend the local coordinates $x^{A}$ from $\partial \widetilde{\mathscr{M}}$ to $\mathscr{O}$ by requiring the $x^{A}$ 's to be constant along the null geodesics $\gamma_{p}$.

3. Let $q \in \mathscr{O}$, then $q$ belongs to some null geodesic $\gamma_{p}$ defined above. We define the function $u$ by letting $u(q)=y^{0}(p)$. In other words, $u$ is defined to be equal to $c$ on $\mathscr{N}_{c}$.

4. Set $x:=\Omega$, the conformal compactifying factor as in (2.1). Passing to a subset of $\mathscr{O}$ if necessary, the functions $\left(u, x, x^{A}\right)$ form a coordinate system on $\mathscr{O}$. By construction the curves $s \mapsto\left(u, x=s, x^{A}\right)$ are null geodesics initially normal to $S_{c}$ :

$$
\widetilde{g}\left(\partial_{x}, \partial_{x}\right)=0,\left.\quad \widetilde{g}\left(\partial_{x}, \partial_{x^{A}}\right)\right|_{x=0}=0 .
$$

We recall the usual calculation, which uses the fact that $\partial_{x}$ is tangent to null geodesics, $\nabla_{\partial_{x}} \partial_{x}=\kappa \partial_{x}$ for some function $\kappa$ :

$$
\begin{aligned}
& \partial_{x}\left(\widetilde{g}\left(\partial_{A}, \partial_{x}\right)\right)=\widetilde{g}\left(\nabla_{\partial_{x}} \partial_{A}, \partial_{x}\right)+\widetilde{g}\left(\partial_{A}, \nabla_{\partial_{x}} \partial_{x}\right) \\
& \quad=\widetilde{g}\left(\nabla_{\partial_{A}} \partial_{x}, \partial_{x}\right)+\kappa \widetilde{g}\left(\partial_{A}, \partial_{x}\right)=\frac{1}{2} \partial_{A}\left(\widetilde{g}\left(\partial_{x}, \partial_{x}\right)\right)+\kappa \widetilde{g}\left(\partial_{A}, \partial_{x}\right) \\
& \quad=\kappa \widetilde{g}\left(\partial_{A}, \partial_{x}\right) .
\end{aligned}
$$

Thus

$$
\partial_{x}\left(\widetilde{g}\left(\partial_{A}, \partial_{x}\right)\right)=\kappa \widetilde{g}\left(\partial_{A}, \partial_{x}\right),
$$

which provides a linear homogeneous ODE in $x$ for $\widetilde{g}\left(\partial_{A}, \partial_{x}\right)$, with vanishing initial data at $x=0$. We conclude that

$$
\widetilde{g}\left(\partial_{x}, \partial_{x}\right)=0, \quad \widetilde{g}\left(\partial_{x}, \partial_{x^{A}}\right)=0 .
$$


Equivalently, the level sets of $u$ are null hypersurfaces generated by the integral curves of $\partial_{x}$.

The Bondi radial coordinate $r_{\mathrm{Bo}}$ (the subscript "Bo" stands for "Bondi") is defined by setting

$$
r_{\mathrm{Bo}}:=\left(\frac{\operatorname{det} g_{A B}}{\operatorname{det} \stackrel{\circ}{g}_{A B}}\right)^{\frac{1}{2(n-1)}}=\frac{1}{x}\left(\frac{\operatorname{det} \widetilde{g}_{A B}}{\operatorname{det} \stackrel{\circ}{g}_{A B}}\right)^{\frac{1}{2(n-1)}},
$$

where $n$ is the space-dimension.

The final coordinate system $\left(u_{\mathrm{Bo}}, r_{\mathrm{Bo}}, x_{\mathrm{Bo}}^{A}\right)$ is obtained by setting, in addition to $(2.6)$,

$$
u_{\mathrm{Bo}}:=u, x_{\mathrm{Bo}}^{A}:=x^{A} \quad \Longrightarrow \quad \partial_{x}=\frac{\partial r_{\mathrm{Bo}}}{\partial x} \partial_{r_{\mathrm{Bo}}}, \partial_{x^{A}}=\partial_{x_{\mathrm{Bo}}^{A}}+\frac{\partial x}{\partial x_{\mathrm{Bo}}^{A}} \partial_{x} .
$$

It follows from (2.5) together with the last implication that

$$
\widetilde{g}\left(\partial_{r_{\mathrm{Bo}}}, \partial_{r_{\mathrm{Bo}}}\right)=0=\widetilde{g}\left(\partial_{r_{\mathrm{Bo}}}, \partial_{x_{\mathrm{Bo}}^{A}}\right), \quad \widetilde{g}\left(\partial_{x^{A}}, \partial_{x^{B}}\right)=\widetilde{g}\left(\partial_{x_{\mathrm{Bo}}^{A}}, \partial_{x_{\mathrm{Bo}}^{B}}\right),
$$

which shows that, on $\mathscr{O}$, the metric satisfies indeed the Bondi conditions

$$
\widetilde{g}_{r_{\mathrm{Bo}} x_{\mathrm{Bo}}^{A}}=0=\widetilde{g}_{r_{\mathrm{Bo}} r_{\mathrm{Bo}}}, \quad \sqrt{\operatorname{det} g_{x_{\mathrm{Bo}}^{A} x_{\mathrm{Bo}}^{B}}}=r^{n-1} \sqrt{\operatorname{det}{\stackrel{\tilde{g}}{x_{\mathrm{Bo}}^{A} x_{\mathrm{Bo}}^{B}}}} .
$$

Equation (2.6) implies that $r_{\text {Bo }}$ has a full asymptotic expansion in terms of powers of $x$ :

$$
r_{\mathrm{Bo}}=\frac{1}{x}+\left(r_{\mathrm{Bo}}\right)_{0}\left(u, x^{A}\right)+\left(r_{\mathrm{Bo}}\right)_{1}\left(u, x^{A}\right) x+\ldots,
$$

where the asymptotic expansion coefficients $\left(r_{\mathrm{Bo}}\right)_{n}$ are functions of $\left(u, x^{A}\right)$. This can be inverted to give a full asymptotic expansion

$$
x=\frac{1}{r_{\mathrm{Bo}}}+\frac{\left(r_{\mathrm{Bo}}\right)_{0}\left(u, x^{A}\right)}{r_{\mathrm{Bo}}^{2}}+\frac{\left(r_{\mathrm{Bo}}\right)_{0}\left(u, x^{A}\right)^{2}+\left(r_{\mathrm{Bo}}\right)_{1}\left(u, x^{A}\right)}{r_{\mathrm{Bo}}^{3}}+\ldots
$$

(Indeed, if we set $y:=1 / r_{\mathrm{Bo}}$, then (2.6) becomes

$$
y=x\left(\frac{\operatorname{det} \stackrel{\circ}{g}_{A B}}{\operatorname{det} \widetilde{g}_{A B}}\right)^{\frac{1}{2(n-1)}},
$$

and existence of a smooth function $x=x(y)$ follows from the implicit function theorem.)

Since all metric functions are smooth in $\left(u, x^{A}, x\right)$, they have complete asymptotic expansions in terms of $1 / r_{\text {Bo }}$, with coefficients depending smoothly upon $\left(u_{\mathrm{Bo}}, x_{\mathrm{Bo}}^{A}\right)$.

As a special case of the construction above, we have proved: 
Proposition 2.1 Let $\mathscr{N}$ be a null hypersurface intersecting smoothly and transversally a section $S$ of conformal infinity in a smoothly conformally compactifiable space-time with cosmological constant $\Lambda \in \mathbb{R}$. There exist adapted coordinates $\left(r, x^{A}\right)$ on $\mathscr{N}$ in which the restrictions $\bar{g}_{A B}$ to $\mathscr{N}$ of the metric functions $g_{A B}$ take the form

$$
\bar{g}_{A B}=r^{2}\left(\stackrel{\stackrel{\check{g}}{g}}{A B}\left(x^{C}\right)+O\left(r^{-1}\right)\right),
$$

with $r^{-2} \bar{g}_{A B}$ having full asymptotic expansions in terms of inverse powers of $r$. These coordinates can be chosen to satisfy the Bondi conditions (2.7) near $\mathscr{N}$. In dimension $3+1$ the metric $\stackrel{\check{g}}{\text { on }} S$ can be arbitrarily chosen, in higher dimension $\sqrt{\operatorname{det} \tilde{g}}$ can be arbitrarily chosen.

In what follows we wish to address two questions:

1. Assuming vacuum, can the expansion above be made more precise? and

2. How to read-off the mass from the above expansions?

For this, some preliminary results will be needed.

Since the case $\Lambda=0$ has been satisfactorily covered elsewhere (cf. [13,17] and references therein), from now on we assume

$$
\Lambda \neq 0 \text {. }
$$

\subsection{Fefferman-Graham expansions}

Recall that smooth conformal compactifiability of a metric satisfying the vacuum Einstein equations implies existence of a coordinate system

$$
\left(x, x^{a}\right) \equiv\left(x, x^{0}, x^{A}\right)
$$

near $\partial \widetilde{\mathscr{M}}$ in which the metric admits a Fefferman-Graham expansion [20,26]: We can write the metric as

$$
g=x^{-2} \ell^{2}\left( \pm d x^{2}+\tilde{g}_{a b}\left(x, x^{c}\right) d x^{a} d x^{b}\right),
$$

where $\ell$ is a constant related to the cosmological constant, and where the sign in front of $d x^{2}$ is the negative of the sign of $\Lambda$. For even values of $n$ we have

$$
\begin{aligned}
\tilde{g}_{a b}\left(x, x^{c}\right)= & \stackrel{\tilde{g}}{a b}_{a b}\left(x^{c}\right)+\left(\tilde{g}_{2}\right)_{a b}\left(x^{c}\right) x^{2}+\ldots+\left(\tilde{g}_{n-2}\right)_{a b}\left(x^{c}\right) x^{n-2} \\
& +\left(\tilde{g}_{n}\right)_{a b}\left(x^{c}\right) x^{n}+\left(\tilde{g}_{\log }\right)_{a b}\left(x^{c}\right) x^{n} \log x+o\left(x^{n}\right) .
\end{aligned}
$$

Here $\partial \widetilde{\mathscr{M}}$ is the zero-level set of $x$, the tensor field

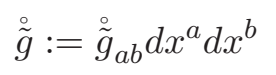

is a representative of the conformal class of metrics induced by $\widetilde{g}$ on $\partial \widetilde{\mathscr{M}}$ (Riemannian if $\Lambda>0$, Lorentzian if $\Lambda<0$ ), and for $i=1, \ldots n-1$ the smooth tensor fields

$$
\tilde{g}_{i}:=\left(\tilde{g}_{i}\right)_{a b} d x^{a} d x^{b} \text { and } \tilde{g}_{\log }:=\left(\tilde{g}_{\log }\right)_{a b} d x^{a} d x^{b}
$$




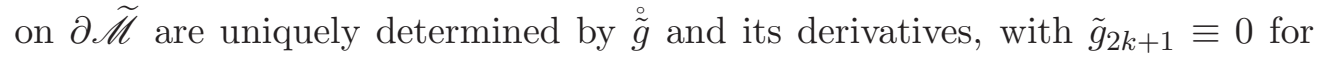
$2 k+1<n$. We will interchangeably write $\left(\tilde{g}_{i}\right)_{a b}$ and $\left(\tilde{g}_{a b}\right)_{i}$ in what follows.

For odd values of $n$ the expansion reads instead

$$
\begin{aligned}
\tilde{g}_{a b}\left(x, x^{c}\right)= & \stackrel{ᄋ}{g}_{a b}\left(x^{c}\right)+\left(\tilde{g}_{2}\right)_{a b}\left(x^{c}\right) x^{2}+\ldots+\left(\tilde{g}_{n-1}\right)_{a b}\left(x^{c}\right) x^{n-1} \\
& +\left(\tilde{g}_{n}\right)_{a b}\left(x^{c}\right) x^{n}+o\left(x^{n}\right),
\end{aligned}
$$

with again $\tilde{g}_{2 k+1} \equiv 0$ for $2 k+1<n$.

We have, both for even and odd $n \geq 3$,

$$
\left(\tilde{g}_{2}\right)_{a b}=-\frac{1}{n-2}\left(\stackrel{\circ}{R}_{a b}-\frac{\stackrel{\circ}{R}}{2(n-1)} \stackrel{\circ}{\tilde{g}}_{a b}\right),
$$

where $\stackrel{\circ}{R}_{a b}$ is the Ricci tensor of the metric $\stackrel{\mathscr{g}}{\text {. }}$

As an example, if $g$ is a Birmingham metric, (6.1) below, with zero mass, we set

$$
\frac{d x}{x}=-\frac{d r}{\ell \sqrt{\frac{r^{2}}{\ell^{2}}+\beta}} \Longrightarrow r=\ell\left(\frac{1}{x}-\frac{\beta x}{4}\right),
$$

where a convenient choice of an integration constant has been made. The metric becomes

$$
g=x^{-2} \ell^{2}\left(d x^{2}-\left(1+\frac{\beta x^{2}}{4}\right)^{2} \ell^{-2} d t^{2}+\left(1-\frac{\beta x^{2}}{4}\right)^{2} \stackrel{\circ}{h}\right) .
$$

In any case, we are led to consider metrics of the form

$$
g=x^{-2} \ell^{2}\left( \pm d x^{2}+\stackrel{\check{g}}{g}+\tilde{g}_{2} x^{2}+O\left(x^{p}\right)\right)
$$

with

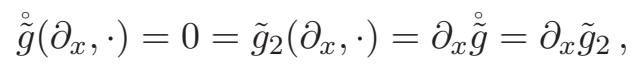

where $p=4$ in dimensions $n \geq 5, p=3$ in dimension $n=3$, and $p$ is any number smaller than four when $n=4$ (in that last dimension $O\left(x^{p}\right)$ with $p<4$ can in fact be replaced by $\left.O\left(x^{4} \ln x\right)\right)$.

\subsection{The next term and the geometry of $\mathscr{N}$}

We will see below that, in a characteristic-Cauchy-problem context, the regularity properties of the space-time metric are determined by the first three coefficients in the expansion (2.10). This raises the question, whether or not conformal smoothness implies that some of those coefficients are zero. The aim of this section is to show that the next-to-leading term in the expansion (2.10) will not vanish in general. This will be done by relating this term to the tracefree part of the extrinsic curvature, within the conformal boundary, of a section $\stackrel{\mathscr{N}}{\text { of }} \partial \tilde{\mathscr{M}}$.

Consider, thus a null hypersurface $\mathscr{N}$ with field of future-directed null tangents $L$ such that the closure $\overline{\mathscr{N}}$ in $\widetilde{\mathscr{M}}$ of $\mathscr{N}$ intersects $\partial \widetilde{\mathscr{M}}$ transversally in a 


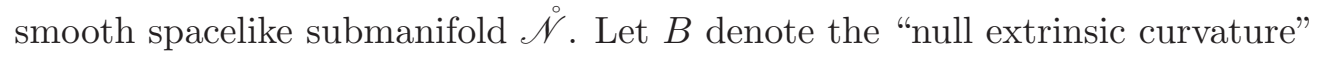
of $\mathscr{N}$,

$$
B(X, Y):=g\left(\nabla_{X} L, Y\right),
$$

defined for $X, Y$ tangent to $\mathscr{N}$. We will invoke the Fefferman-Graham expansions, and the law of conformal transformations of the objects involved.

In what follows we use the notation of [7, Appendix A]. From that last reference we have

$$
\bar{\Gamma}_{A B}^{0}=-\frac{1}{2} \nu^{0} \partial_{1} \bar{g}_{A B}
$$

Hence, when $L=\partial_{r}$,

$$
\begin{aligned}
B_{A B} & =g\left(\nabla_{A} L, \partial_{B}\right)=-g\left(\nabla_{A} \partial_{B}, L\right)=-g\left(\nabla_{A} \partial_{B}, \partial_{r}\right) \\
& =-g_{\mu r} \Gamma_{A B}^{\mu}=-g_{0 r} \Gamma_{A B}^{0}=-\nu_{0} \Gamma_{A B}^{0}=\frac{1}{2} \partial_{1} \bar{g}_{A B} \\
& =\chi_{A B},
\end{aligned}
$$

with $\chi$ as in (3.8) below.

Let

$$
\widetilde{g}=\Omega^{2} g
$$

be the unphysical conformally rescaled metric. Let $\mathscr{I} \equiv \partial \widetilde{\mathscr{M}}$ be the conformal boundary, which in vacuum is spacelike if $\Lambda>0$ and timelike if $\Lambda<0$. In what follows we will assume that $\Lambda<0$; the argument applies to the case $\Lambda>0$ after obvious modifications.

Let $\tilde{N}$ be the inwards-directed $\widetilde{g}$-unit normal to $\mathscr{I}$. Let $\mathscr{S}$ be a smooth spacelike hypersurface in $\widetilde{\mathscr{M}}$ meeting $\mathscr{I}$ orthogonally at $\mathscr{\mathscr { N }}$. Let $\tilde{T}$ denote a future-directed $\widetilde{g}$-unit normal to $\mathscr{S}$. Let $\tilde{L}$ be a smooth-up-to-boundary field of tangents of generators of $\bar{N}$. There exists a strictly positive function $\omega$ so that

$$
\tilde{L}=\omega(\tilde{T}-\tilde{N}) \text { at } \stackrel{\circ}{N} .
$$

Here $\mathscr{N}$ is thought to lie to the past of $\mathscr{S}$ and is thus the boundary of the past domain of dependence of $\mathscr{S}$ in the unphysical, conformally rescaled space-time (and hence also in the physical space-time).

Let $x$ be a defining function for $\mathscr{I}$, and let the conformal factor be $\Omega=x$. Using $r_{\mathrm{Bo}} \approx 1 / x$ (compare (2.8)) as the parameter along the generators in the physical space-time, with $L=\partial_{r_{\mathrm{Bo}}}$, we see that the function $\omega$ in $(2.23)$ can be chosen so that

$$
L=\Omega^{2} \tilde{L},
$$

and note that with this choice the vector field $\tilde{L}$ extends smoothly across the conformal boundary $\{x=0\}$. Letting $\tilde{\chi}_{A B}$ denote the corresponding "unphysical $\chi$-tensor" of $\mathscr{N}$, we have

$$
\begin{aligned}
\tilde{\chi}_{A B} & =\tilde{B}_{A B}=-\widetilde{g}\left(\widetilde{\nabla}_{A} \partial_{B}, \tilde{L}\right) \\
& =-\Omega^{2} g\left(\nabla_{A} \partial_{B}+\frac{1}{\Omega}\left(\nabla_{A} \Omega \partial_{B}+\nabla_{B} \Omega \partial_{A}-g_{A B} \nabla \Omega\right), \tilde{L}\right) \\
& =-g\left(\nabla_{A} \partial_{B}+\frac{1}{\Omega}\left(\nabla_{A} \Omega \partial_{B}+\nabla_{B} \Omega \partial_{A}-g_{A B} \nabla \Omega\right), L\right) \\
& =\chi_{A B}+\frac{1}{\Omega} L(\Omega) g_{A B} .
\end{aligned}
$$


On the other hand, on $\stackrel{\mathscr{N}}{\text { it holds that }}$

$$
\left.\tilde{\chi}_{A B}\right|_{\mathscr{N}}=-\widetilde{g}\left(\widetilde{\nabla}_{A} \partial_{B}, \omega(\tilde{T}-\tilde{N})\right)=\omega\left(\tilde{K}_{A B}-\tilde{H}_{A B}\right)
$$

where $\tilde{H}$ is the extrinsic curvature tensor of $\mathscr{I}$ in $(\mathscr{M}, \widetilde{g})$, and $\tilde{K}$ is that of $\mathscr{S}$.

The Fefferman-Graham expansion shows that the trace-free part of $\tilde{H}$ vanishes at $\mathscr{I}$, so that

$$
\left.\tilde{\chi}_{A B}\right|_{\mathscr{N}}=\left.\omega \tilde{K}_{A B}\right|_{\{x=0\}} .
$$

For further reference we note that the trace-free part of $\tilde{H}$ is in fact $O\left(x^{2}\right)$ when a) $\mathscr{I}$ is locally conformally flat, or when b)

$$
\stackrel{\circ}{R}_{A B} \text { is proportional to } \stackrel{\circ}{g}_{A B} \text {. }
$$

In order to determine $\tilde{K}$ we use the coordinates of (2.12)-(2.13). In these coordinates let $\mathscr{S}$ be given by the equation

$$
x^{0}=f\left(x, x^{A}\right)=f_{0}\left(x^{A}\right)+x f_{1}\left(x^{A}\right)+O\left(x^{2}\right),
$$

with smooth functions $f_{0}, f_{1}$ (in fact, $f_{1}$ vanishes if $\mathscr{S}$ meets the boundary orthogonally, but this is not needed for our conclusions below), then

$$
\tilde{T}=\varepsilon \tilde{\alpha}^{-2}\left(d x^{0}-\partial_{A} f_{0} d x^{A}-f_{1} d x+O(x)\right),
$$

where $\tilde{\alpha}$ is determined by the condition $\widetilde{g}(\tilde{T}, \tilde{T})=\varepsilon \in\{ \pm 1\}$ :

$$
\tilde{\alpha}^{2}=\varepsilon\left(\tilde{\tilde{g}}^{00}-2 \tilde{\tilde{g}}^{0 A} \partial_{A} f_{0}+\stackrel{\check{g}}{A B}^{A B} \partial_{A} f_{0} \partial_{B} f_{0}+f_{1}^{2}+O(x)\right) .
$$

We emphasise that if the intersection of $\mathscr{S}$ with $\mathscr{I}$ is a smooth spacelike submanifold of $\mathscr{I}$, as assumed here, then both $\tilde{\alpha}$ and $\tilde{\alpha}^{-1}$ are smooth.

We will denote by $e_{A}$ the tangential lift of $\partial_{A}$ to the graph of $f$ :

$$
e_{A}=\partial_{A}+\partial_{A} f \partial_{0}=: e_{A}^{\mu} \partial_{\mu}
$$

For small $x$ the metric $\widetilde{g}:=x^{2} g$ behaves as

$$
\widetilde{g} \rightarrow_{x \rightarrow 0} \stackrel{\check{g}}{:}=\ell^{2}\left(d x^{2}+\stackrel{\check{g}}{a b}_{a b}\left(x^{c}\right) d x^{a} d x^{b}\right) .
$$

The Christoffel symbols $\stackrel{\stackrel{\Gamma}{\Gamma}}{\beta \gamma}^{\alpha}$ of the asymptotic metric $\stackrel{\circ}{g}$ read

$$
\stackrel{\circ}{\Gamma}_{a b}^{c} \equiv \Gamma[\tilde{g}]_{a b}^{c}=: \stackrel{\circ}{\Gamma}_{a b}^{c}, \quad \stackrel{\circ}{\Gamma}_{\mu \nu}^{1}=\stackrel{\circ}{\Gamma}_{1 \nu}^{\mu}=0
$$

This can be used to determine the asymptotic behaviour of $\tilde{K}_{A B}$ :

$$
\begin{aligned}
\tilde{K}_{A B}= & -\widetilde{g}\left(\widetilde{\nabla}_{e_{A}} e_{B}, \tilde{T}\right)=-\left(e_{A}\left(e_{B}{ }^{\mu}\right)+\widetilde{\Gamma}_{\alpha \beta}^{\mu} e_{A}{ }^{\alpha} e_{B}{ }^{\beta}\right) \tilde{T}_{\mu} \\
= & -\varepsilon \tilde{\alpha}^{-2}(\underbrace{\partial_{A} \partial_{B} f_{0}-\stackrel{\circ}{\Gamma}_{A B}^{C} \partial_{C} f_{0}}_{=: \mathscr{\mathscr { D }}_{A} \mathscr{\mathscr { D }}_{B} f_{0}}+\stackrel{\circ}{\Gamma}_{A B}^{0}+2\left(\stackrel{\circ}{\Gamma}_{0(A}^{0}-\partial_{C} f_{0} \stackrel{\circ}{\Gamma}_{0(A}^{C}\right) \partial_{B} f_{0} \\
& \left.+\left(\stackrel{\circ}{\Gamma}_{00}^{0}-\stackrel{\circ}{\Gamma}_{00}^{C} \partial_{C} f_{0}\right) \partial_{A} f_{0} \partial_{B} f_{0}\right)+O(x) \\
= & \stackrel{\check{K}}{K}_{A B}+O(x),
\end{aligned}
$$


where

$$
\stackrel{\stackrel{\check{K}}{K}}{A B}:=\left.\tilde{K}_{A B}\right|_{x=0} \cdot
$$

Using (2.25) and (2.27) we obtain

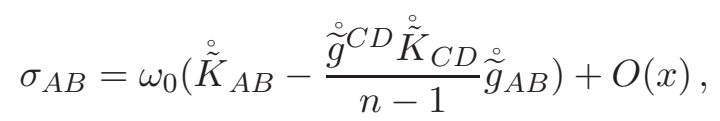

where $\omega_{0}:=\left.\omega\right|_{x=0}$.

We conclude that on characteristic hypersurfaces smoothly meeting $\mathscr{I}$ we have $\sigma_{A B}=O(1)$ for large $r$, with

$$
\left.\sigma\right|_{x=0} \text { being non-zero in general. }
$$

As an example, consider the case $\Lambda<0$ with

a metric $\left.\widetilde{g}_{a b}\right|_{x=0}$ which is static up to a conformal factor.

We can then rescale the metric, and adjust the $x$-coordinate accordingly, so that $\left.\widetilde{g}_{a b}\right|_{x=0}$ is in fact $x^{0}$-independent. A further, $x^{0}$-independent, rescaling can be done so that $\widetilde{g}_{00}$ is constant. We can further choose manifestly static local coordinates, where by definition $\left.\widetilde{g}_{A 0}\right|_{x=0}=0$ (this can be done globally when $\widetilde{g}_{A 0} d x^{A}$ is exact, which will certainly be the case if $\mathscr{N}$ is simply connected). Setting $X=\partial_{0}$ and using

$$
\partial_{0} \widetilde{g}_{a b}=\mathscr{L}_{\partial_{0}} \widetilde{g}_{a b}=\widetilde{\nabla}_{a} X_{b}+\widetilde{\nabla}_{b} X_{a}=-2 \widetilde{\Gamma}_{a b}^{0},
$$

we see that all the $\stackrel{\circ}{\Gamma}_{a b}^{0}$ 's vanish, and in fact $\stackrel{\circ}{\Gamma}_{0 b}^{a}=0$. With these choices we will have

$$
\stackrel{\circ}{\tilde{K}}_{A B} \sim \stackrel{\circ}{g}_{A B}
$$

if and only if

$$
\stackrel{\mathscr{D}}{A}_{A} \stackrel{\mathscr{D}}{B}_{B} f_{0}-\frac{\stackrel{\mathscr{D}}{C}^{C} \stackrel{\mathscr{D}}{C}_{C} f_{0}}{n-1} \stackrel{\check{g}}{A B}_{A B}=0 .
$$

We conclude that under (2.35) and (2.37) we have

$$
\sigma_{A B}=O\left(r^{-1}\right) \quad \Longleftrightarrow \quad|\sigma|^{2}=O\left(r^{-6}\right)
$$

for large $r$.

We also see that the (2.36) is a necessary and sufficient condition for (2.38) in any case.

\section{Characteristic hypersurfaces}

Throughout this section we allow arbitrary space-time dimension $n+1 \geq 4$.

As a first step towards understanding the mass of characteristic hypersurfaces, a review of the characteristic Cauchy problem for the Einstein equations is in order. 


\subsection{Wave-map gauge}

Let, thus $\mathscr{N}$ be a characteristic hypersurface. Following [7], we split the Einstein equations along $\mathscr{N}$ into constraint and evolution equations using the generalized wave-map gauge $[7,21]$, which is characterized by the vanishing of the generalized wave-gauge vector:

$$
H^{\lambda}=0
$$

which is defined as

$$
H^{\lambda}:=\Gamma^{\lambda}-V^{\lambda}, \text { where } V^{\lambda}:=\hat{\Gamma}^{\lambda}+W^{\lambda}, \quad \Gamma^{\lambda}:=g^{\alpha \beta} \Gamma_{\alpha \beta}^{\lambda}, \quad \hat{\Gamma}^{\lambda}:=g^{\alpha \beta} \hat{\Gamma}_{\alpha \beta}^{\lambda} .
$$

Here $\hat{\Gamma}$ are the Christoffel symbols of an auxiliary target space metric $\hat{g}$, which can be chosen as convenient for the problem at hand. The gauge source functions $W^{\lambda}=W^{\lambda}\left(x^{\mu}, g_{\mu \nu}\right)$ can be freely specified and are allowed to depend upon the coordinates chosen and the metric itself, but not upon derivatives thereof. In (3.2) and in what follows we decorate objects associated with the target metric $\hat{g}$ with the hat symbol "^»".

\subsection{Characteristic surfaces, adapted null coordinates and as- sumptions on the metric}

It is convenient to use coordinates adapted to the characteristic surface, called adapted null coordinates $\left(x^{0}=u, x^{1}=r, x^{A}\right), A \in\{2, \ldots, n\}$. The coordinate $r>r_{0} \geq 0$, where we allow a boundary or a vertex at a value $r=r_{0}$ possibly different from zero, parametrizes the null geodesics issuing from $\left\{r_{0}\right\}$ and generating the null hypersurface, which coincides with the set $\{u=0\}$. The $x^{A}$ 's are local coordinates on the level sets $\{u=0, r=$ const. $\}$. The trace of the metric on the characteristic surface can then be written as (we will interchangeably use $x^{0}$ and $u$ )

$$
\bar{g}=\bar{g}_{\mu \nu} d x^{\mu} d x^{\nu}=\bar{g}_{00}(d u)^{2}+2 \nu_{0} d u d r+2 \nu_{A} d u d x^{A}+\check{g},
$$

where we use the notation

$$
\nu_{0}:=\bar{g}_{0 r}, \quad \nu_{A}:=\bar{g}_{0 A}, \quad \check{g}:=\bar{g}_{A B} d x^{A} d x^{B} .
$$

Here and throughout an overline denotes the restriction of a space-time object to $\mathscr{N}$.

Under the hypotheses of Proposition 2.1, for $r$ large we can write

$$
\bar{g}_{A B}=\stackrel{\circ}{h}_{A B} r^{2}+\left(\bar{g}_{A B}\right)_{-1} r+\left(\bar{g}_{A B}\right)_{0}+O\left(r^{-1}\right),
$$

where we use the symbol $\stackrel{\circ}{h}$ to denote the standard metric on the boundary manifold, and $\left(\bar{g}_{A B}\right)_{-i}=\left(\bar{g}_{A B}\right)_{-i}\left(x^{C}\right), i \in \mathbb{N}$, are some smooth tensors on that manifold. We also require that $O\left(r^{-1}\right)$ terms remain $O\left(r^{-1}\right)$ under $x^{C}$. differentiation, and become $O\left(r^{-2}\right)$ under $r$-differentiation; similarly for $O\left(r^{-n}\right)$.

The restriction of the inverse metric to $\mathscr{N}$ takes the form

$$
\bar{g}^{\#}=2 \nu^{0} \partial_{u} \partial_{r}+\bar{g}^{r r} \partial_{r} \partial_{r}+2 \bar{g}^{r A} \partial_{r} \partial_{A}+\bar{g}^{A B} \partial_{A} \partial_{B},
$$


where $\bar{g}^{A B}$ is the inverse of $\bar{g}_{A B}$ and

$$
\nu^{0}:=\bar{g}^{0 r}=\frac{1}{\nu_{0}}, \quad \bar{g}^{r A}=-\nu^{0} \nu^{A}=-\nu^{0} \bar{g}^{A B} \nu_{B}, \quad \bar{g}^{r r}=\left(\nu^{0}\right)^{2}\left(\nu^{A} \nu_{A}-\bar{g}_{00}\right) .
$$

The null second fundamental form of $\mathscr{N}$ is intrinsically defined and does not depend on transverse derivatives of the metric, see (2.20). In adapted null coordinates it reads (compare (2.22))

$$
\chi_{A B}=\frac{1}{2} \partial_{r} \bar{g}_{A B} .
$$

The expansion, also called divergence, of the characteristic surface will be denoted by

$$
\tau:=\chi_{A}{ }^{A}=\bar{g}^{A B} \chi_{A B},
$$

while the trace-free part of the null second fundamental form

$$
\sigma_{A}^{B}=\chi_{A}^{B}-\frac{1}{2} \tau \delta_{A}^{B}
$$

is called the shear of $\mathscr{N}$.

The constraint equations for the characteristic problem will be referred to as Einstein wave-map gauge constraints. In space-time dimension $n+1 \geq 3$ they read [7]

$$
\begin{aligned}
\left(\partial_{r}-\kappa\right) \tau+\frac{1}{n-1} \tau^{2}= & -|\sigma|^{2}-8 \pi \bar{T}_{r r} \\
\left(\partial_{r}+\frac{1}{2} \tau+\kappa\right) \nu^{0}= & -\frac{1}{2} \bar{V}^{0} \\
\left(\partial_{r}+\tau\right) \xi_{A}= & 2 \check{\nabla}_{B} \sigma_{A}^{B}-2 \frac{n-2}{n-1} \partial_{A} \tau-2 \partial_{A} \kappa-16 \pi \bar{T}_{r A}, \\
\left(\partial_{r}+\frac{1}{2} \nu_{0} \bar{V}^{0}\right) \nu^{A}= & \frac{1}{2} \nu_{0}\left(\bar{V}^{A}-\xi^{A}-\bar{g}^{B C} \check{\Gamma}_{B C}^{A}\right) \\
\left(\partial_{r}+\tau+\kappa\right) \zeta= & \frac{1}{2}|\xi|^{2}-\check{\nabla}_{A} \xi^{A}-\check{R} \\
& +\underbrace{8 \pi\left(\bar{g}^{A B} \bar{T}_{A B}-\bar{T}\right)}_{=: S}+2 \Lambda \\
\left(\partial_{r}+\frac{1}{2} \tau+\kappa\right) \bar{g}^{r r}= & \frac{1}{2} \zeta-\bar{V}^{r},
\end{aligned}
$$

where

$$
|\sigma|^{2}:=\sigma_{A}^{B}{\sigma_{B}}^{A}, \quad|\xi|^{2}:=\bar{g}^{A B} \xi_{A} \xi_{B}, \quad \xi^{A}:=\bar{g}^{A B} \xi_{B}, \quad \bar{T}:=\bar{g}^{\mu \nu} \bar{T}_{\mu \nu} .
$$

All objects associated with the one-parameter family of Riemannian metrics $\check{g}$ are decorated with the check symbol " $\sim$ ". The boundary conditions needed to integrate (3.11)-(3.16) starting from a light-cone vertex $r_{0}=0$ follow from the requirement of smoothness of the metric there, see [7, Section 4.5]. 
The function $\kappa$ is defined through the equation

$$
\nabla_{\partial_{r}} \partial_{r}=\kappa \partial_{r}
$$

and reflects the freedom to choose the coordinate $r$ which parametrizes the null geodesic generators of $\mathscr{N}$. The "auxiliary" fields $\xi_{A}$ and $\zeta$ have been introduced to transform (3.11)-(3.16) into first-order equations. The field $\xi_{A} \equiv-2 \bar{\Gamma}_{r A}^{r}$ represents connection coefficients, while the field $\zeta$ is the divergence of the family of suitably normalized null generators normal to the spheres of constant radius $r$ and transverse to the characteristic surface. In coordinates adapted to the lightcone as in [7] the space-time formula for $\zeta$ reads (compare [7, Equations (10.32) and (10.36)]; note, however, that there is a term $\tau \bar{g}^{11} / 2$ missing at the righthand side of the second equality in (10.36) there)

$$
\zeta:=\left(2 \partial_{r}+2 \kappa+\tau\right) \bar{g}^{r r}+2 \bar{\Gamma}^{r} \equiv 2 \bar{g}^{A B} \bar{\Gamma}_{A B}^{r}+\tau \bar{g}^{r r} .
$$

To integrate the wave-map gauge constraints (3.11)-(3.16) one also needs the components $\bar{V}^{\mu}$, which are determined by the wave-map gauge (3.1)-(3.2). We have, in adapted null coordinates [7, Appendix A, Equations (A.29)-(A.31)],

$$
\begin{aligned}
\bar{\Gamma}^{0} \equiv \bar{g}^{\lambda \mu} \bar{\Gamma}_{\lambda \mu}^{0} \equiv & \nu^{0}\left(\nu^{0} \overline{\partial_{0} g_{11}}-\tau\right) \\
\bar{\Gamma}^{1} \equiv \bar{g}^{\lambda \mu} \bar{\Gamma}_{\lambda \mu}^{1} \equiv & -\partial_{1} \bar{g}^{11}+\bar{g}^{11} \nu^{0}\left(\frac{1}{2} \overline{\partial_{0} g_{11}}-\partial_{1} \nu_{0}-\tau \nu_{0}\right) \\
& +\nu^{0} \bar{g}^{A B} \check{\nabla}_{B} \nu_{A}-\frac{1}{2} \nu^{0} \bar{g}^{A B} \overline{\partial_{0} g_{A B}} \\
\bar{\Gamma}^{A} \equiv \bar{g}^{\lambda \mu} \bar{\Gamma}_{\lambda \mu}^{A} \equiv & \nu^{0} \nu^{A}\left(\tau-\nu^{0} \overline{\partial_{0} g_{11}}\right)+\nu^{0} \bar{g}^{A B}\left(\overline{\partial_{0} g_{1 B}}+\partial_{1} \nu_{B}-\partial_{B} \nu_{0}\right) \\
& -2 \nu^{0} \nu^{B} \chi_{B}{ }^{A}+\check{\Gamma}^{A}
\end{aligned}
$$

Now, from the restriction to $\mathscr{N}$ of (3.1) and together with the first equation of (3.2) one finds that the choice of the target metric only redefines the fields $\overline{\hat{\Gamma}}^{\mu}$ and $\bar{W}^{\mu}$ entering in the definition of $\bar{V}^{\mu}=\overline{\hat{\Gamma}}^{\mu}+\bar{W}^{\mu}$ without changing $\bar{V}^{\mu}$ itself. Therefore only $\bar{V}^{\mu}$ enters in the Einstein wave-map gauge constraint equations, and so only the explicit form of those fields is relevant in the equations of interest to us.

An adapted coordinate system on a characteristic surface $\mathscr{N}$ will be called Bondi type if the coordinates satisfy Bondi conditions on $\mathscr{N}$, but not necessarily away from $\mathscr{N}$, reserving the name Bondi coordinates for coordinate systems which satisfy Bondi's condition everywhere.

We will start by deriving the asymptotic expansions of all relevant fields in Bondi-type coordinates on the characteristic surface; it appears that the calculations are simplest in those coordinates. We have [29, Equation (5.5)]

$$
\varphi^{\mathrm{Bo}}=r_{\mathrm{Bo}}, \quad \overline{\partial_{0} g_{r r}^{\mathrm{Bo}}}=0, \quad \overline{\partial_{0} g_{r A}^{\mathrm{Bo}}}=0, \quad \bar{g}_{\mathrm{Bo}}^{A B} \overline{\partial_{0} g_{A B}^{\mathrm{Bo}}}=0,
$$

where $\varphi$ is defined by $\tau=2 \partial_{r} \log \varphi$, as well as

$$
\begin{aligned}
& \bar{V}_{\mathrm{Bo}}^{0}=-\tau^{\mathrm{Bo}} \nu_{\mathrm{Bo}}^{0}, \\
& \bar{V}_{\mathrm{Bo}}^{A}=\bar{g}_{\mathrm{Bo}}^{C D}\left(\check{\Gamma}^{\mathrm{Bo}}\right)_{C D}^{A}-\nu_{\mathrm{Bo}}^{0} \check{\nabla}^{A} \nu_{0}^{\mathrm{Bo}}+\nu_{\mathrm{Bo}}^{0}\left(\partial_{r_{\mathrm{Bo}}}+\tau^{\mathrm{Bo}}\right) \nu_{\mathrm{Bo}}^{A}, \\
& \bar{V}_{\mathrm{Bo}}^{r}=\nu_{\mathrm{Bo}}^{0} \check{\nabla}_{A} \nu_{\mathrm{Bo}}^{A}-\left(\partial_{r_{\mathrm{Bo}}}+\tau^{\mathrm{Bo}}+\nu_{\mathrm{Bo}}^{0} \partial_{r_{\mathrm{Bo}}} \nu_{0}^{\mathrm{Bo}}\right) \bar{g}_{\mathrm{Bo}}^{r r} .
\end{aligned}
$$


As mentioned previously the Einstein wave-map gauge constraints form a hierarchical system of ODEs along the null generators of the characteristic surface which can be solved step-by-step.

\section{Asymptotic solutions of the characteristic wave- map gauge constraints, $\Lambda \neq 0$}

Throughout this section we assume that the space-dimension $n=3$.

In [28] asymptotic solutions of the Einstein wave-map gauge constraints (3.11)-(3.16) with $\Lambda=0$ have been obtained in the form of polyhomogeneous expansions of the solution at infinity, i.e., expansions in terms of inverse powers of $r$ and of powers of $\log r$. Our aim is to obtain similar expansions when $\Lambda \neq 0$, with the goal to find a formula for the characteristic mass.

We will assume that for large $r$

$$
\sigma_{A}^{\mathrm{Bo}}{ }_{A}^{B}=\left(\sigma_{A}^{\mathrm{Bo}}{ }_{A}^{B}\right)_{2} r_{\mathrm{Bo}}^{-2}+\left(\sigma_{A}^{\mathrm{Bo}}{ }_{A}^{B}\right)_{3} r_{\mathrm{Bo}}^{-3}+O\left(r_{\mathrm{Bo}}^{-4}\right),
$$

which is compatible with, and more general than, Proposition 2.1. Here $\sigma^{\text {Bo }}$ is the shear of $\mathscr{N}$ in Bondi coordinates, with $\sigma^{\mathrm{Bo}}{ }_{A}^{B}:=\bar{g}^{B C} \sigma^{\mathrm{Bo}}\left(\partial_{A}, \partial_{C}\right)$. As already mentioned, wherever needed in the calculations that follow we will assume that differentiation of error terms $O\left(r^{\alpha}\right)$ with respect to angles preserves the $O\left(r^{\alpha}\right)$ behaviour, while differentiation with respect to $r$ produces terms which are $O\left(r^{\alpha-1}\right)$.

(It follows from our calculations below that the hypothesis (4.1) is equivalent to

$$
\sigma_{A}^{B}=\left(\sigma_{A}^{B}\right)_{2} r^{-2}+\left(\sigma_{A}^{B}\right)_{3} r^{-3}+O\left(r^{-4}\right),
$$

where $r$ is an affine coordinate along the generators of $\mathscr{N}$.)

\subsection{Matter fields}

We start by analyzing the influence of the matter fields on the asymptotic expansion of the metric in Bondi-type coordinates. Our aim is to determine a decay rate of the energy-momentum tensor which is compatible with finite total mass. The decay rates for various components of the energy-momentum tensor will be chosen so that they do not affect the leading-order behavior, as arising in the vacuum case, of the solutions of the equations in which they appear.

For the convenience of the reader we repeat here the relevant equations in Bondi-type gauge (see [29, Equations (5.11)-(5.15)] with the contribution from the cosmological constant $\Lambda$ added here):

$$
\begin{aligned}
\kappa^{\mathrm{Bo}}-\frac{1}{2} r_{\mathrm{Bo}}\left(\left|\sigma^{\mathrm{Bo}}\right|^{2}+8 \pi \bar{T}_{r r}^{\mathrm{Bo}}\right) & =0, \\
\left(\partial_{r_{\mathrm{Bo}}}+\frac{r_{\mathrm{Bo}}}{2}\left(\left|\sigma^{\mathrm{Bo}}\right|^{2}+8 \pi \bar{T}_{r r}^{\mathrm{Bo}}\right)\right) \nu_{\mathrm{Bo}}^{0} & =0, \\
\left(\partial_{r_{\mathrm{Bo}}}+\tau^{\mathrm{Bo}}\right) \xi_{A}^{\mathrm{Bo}}-2 \check{\nabla}_{B} \sigma_{A}^{\mathrm{Bo} B}+\partial_{A} \tau^{\mathrm{Bo}}+r_{\mathrm{Bo}} \partial_{A}\left(\left|\sigma^{\mathrm{Bo}}\right|^{2}+8 \pi \bar{T}_{r r}^{\mathrm{Bo}}\right) & =-16 \pi \bar{T}_{r A}^{\mathrm{Bo}}, \\
\partial_{r_{\mathrm{Bo}}} \nu_{\mathrm{Bo}}^{A}+\left(\check{\nabla}^{A}+\xi_{\mathrm{Bo}}^{A}\right) \nu_{0}^{\mathrm{Bo}} & =0,
\end{aligned}
$$




$$
\begin{aligned}
\left(\partial_{r_{\mathrm{Bo}}}+\tau^{\mathrm{Bo}}+\frac{r_{\mathrm{Bo}}}{2}\left(\left|\sigma^{\mathrm{Bo}}\right|^{2}+8 \pi \bar{T}_{r r}^{\mathrm{Bo}}\right)\right) \zeta^{\mathrm{Bo}}+\check{R}^{\mathrm{Bo}}-\frac{\left|\xi^{\mathrm{Bo}}\right|^{2}}{2}+\check{\nabla}_{A} \xi_{\mathrm{Bo}}^{A}= & \underbrace{8 \pi\left(\bar{g}_{\mathrm{Bo}}^{A B} \bar{T}_{A B}^{\mathrm{Bo}}-\bar{T}^{\mathrm{Bo}}\right)}_{S^{\mathrm{Bo}}}+2 \Lambda, \\
\bar{g}_{\mathrm{Bo}}^{r r}+\left(\tau^{\mathrm{Bo}}\right)^{-1}\left(\zeta^{\mathrm{Bo}}-2 \nu_{\mathrm{Bo}}^{0} \check{\nabla}_{A} \nu_{\mathrm{Bo}}^{A}\right) & =0 .
\end{aligned}
$$

In Bondi-type coordinates, the relation

$$
\tau^{\mathrm{Bo}}=\frac{2}{r_{\mathrm{Bo}}}
$$

is independent of the cosmological constant and of matter fields.

It follows from (4.3), which can be solved algebraically for $\kappa^{\text {Bo }}$, that a term $O\left(r_{\mathrm{Bo}}^{-\alpha_{r r}}\right)$ in $\bar{T}_{r r}^{\mathrm{Bo}}$ with $\alpha_{r r}>2$ produces an $O\left(r_{\mathrm{Bo}}^{-\alpha_{r r}+1}\right)$ term in $\kappa^{\text {Bo }}$ (see also the discussion in Section 4.2 and Equation (4.21)):

$$
\bar{T}_{r r}^{\mathrm{Bo}}=O\left(r_{\mathrm{Bo}}^{-\alpha_{r r}}\right), \alpha_{r r}>2 \quad \Longrightarrow \quad \kappa^{\mathrm{Bo}}=\left(\kappa^{\mathrm{Bo}}\right)_{\text {vacuum }}+O\left(r_{\mathrm{Bo}}^{-\alpha_{r r}+1}\right) .
$$

Next, from (4.4) we find

$$
\nu_{\mathrm{Bo}}^{0}=\left(\nu_{\mathrm{Bo}}^{0}\right)_{\text {vacuum }}+O\left(r_{\mathrm{Bo}}^{-\alpha_{r r}+2}\right) .
$$

In the $\xi_{A}^{\text {Bo }}$-constraint equation (4.5), the assumption

$$
\bar{T}_{r A}^{\mathrm{Bo}}=O\left(r_{\mathrm{Bo}}^{-\alpha_{r A}+1}\right), 4 \neq \alpha_{r A}, 4 \neq \alpha_{r r},
$$

leads to

$$
\xi_{A}^{\mathrm{Bo}}=\left(\xi_{A}^{\mathrm{Bo}}\right)_{\text {vacuum }}+O\left(r_{\mathrm{Bo}}^{-\alpha_{r A}+2}\right)+O\left(r_{\mathrm{Bo}}^{-\alpha_{r r}+2}\right),
$$

where the values $\alpha_{r A}=4$ and $\alpha_{r r}=4$ have been excluded to avoid here a supplementary annoying discussion of logarithmic terms (note that the logarithmic terms will be discussed in detail in the sections that follow):

$$
\begin{aligned}
& \alpha_{r A}=4 \text { or } \alpha_{r r}=4 \Longrightarrow \\
& \quad \xi_{A}^{\mathrm{Bo}}=\left(\xi_{A}^{\mathrm{Bo}}\right)_{\text {vacuum }}+O\left(r_{\mathrm{Bo}}^{-\alpha_{r A}+2}\right)+O\left(r_{\mathrm{Bo}}^{-\alpha_{r r}+2}\right)+O\left(r_{\mathrm{Bo}}^{-2} \log r_{\mathrm{Bo}}\right) .
\end{aligned}
$$

From now on we assume (4.11). To preserve the vacuum asymptotics $\xi_{A}^{\mathrm{Bo}}=$ $O\left(r_{\mathrm{Bo}}^{-1}\right)$ we will moreover require

$$
\alpha_{r r}>3, \quad \alpha_{r A}>3 .
$$

(Anticipating, we have excluded the case $\alpha_{r r}=3$, which introduces $1 / r_{\mathrm{Bo}}$ terms in $\nu_{\mathrm{Bo}}^{0}$, which lead subsequently to logarithmically divergent terms in $\nu_{\mathrm{Bo}}^{A}$. We further note that $\alpha_{r A}=3$ will produce an additional $\left(\xi_{A}^{\mathrm{Bo}}\right)_{1}$-term that would not integrate away in $m_{\mathrm{TB}}$ and would remain as a supplementary $\left(\bar{T}_{r A}^{\mathrm{Bo}}\right)_{2}$-term in our final mass identity (5.56) below.)

Integration of (4.6) gives

$$
\begin{aligned}
\nu_{\mathrm{Bo}}^{A} & =\left(\nu_{\mathrm{Bo}}^{A}\right)_{\text {vacuum }}+O\left(r_{\mathrm{Bo}}^{-\alpha_{r A}+1}\right)+O\left(r_{\mathrm{Bo}}^{-\alpha_{r r}+1}\right) \\
& \Longleftrightarrow \nu_{A}^{\mathrm{Bo}}=\left(\nu_{A}^{\mathrm{Bo}}\right)_{\text {vacuum }}+O\left(r_{\mathrm{Bo}}^{-\alpha_{r A}+3}\right)+O\left(r_{\mathrm{Bo}}^{-\alpha_{r r}+3}\right) .
\end{aligned}
$$


Finally, the asymptotic behavior $\xi_{A}^{\mathrm{Bo}}=O\left(r_{\mathrm{Bo}}^{-1}\right)$ together with (4.7) and (4.8) show that: a term $O\left(r_{\mathrm{Bo}}^{-\alpha_{S}}\right)$ in $S^{\mathrm{Bo}}$ with $\alpha_{S}<2$ would change the leading order behavior of $\zeta^{\mathrm{Bo}} ; \alpha_{S}=2$ would change the leading order term of $\zeta^{\mathrm{Bo}} ; \alpha_{S}=3$ would lead to a logarithmic term in $\zeta^{\text {Bo }}$. This leads to

$$
\begin{aligned}
& S^{\mathrm{Bo}}= O\left(r_{\mathrm{Bo}}^{-\alpha_{S}}\right), \alpha_{S}>3, \alpha_{r r} \neq 5, \\
& \Longrightarrow \quad \zeta^{\mathrm{Bo}}=\left(\zeta^{\mathrm{Bo}}\right)_{\text {vacuum }}+O\left(r_{\mathrm{Bo}}^{-\alpha_{S}+1}\right)+O\left(r_{\mathrm{Bo}}^{-\alpha_{r A}+1}\right)+O\left(r_{\mathrm{Bo}}^{-\alpha_{r r}+3}\right), \\
& \quad \bar{g}_{\mathrm{Bo}}^{r r}=\left(\bar{g}_{\mathrm{Bo}}^{r r}\right)_{\text {vacuum }}+O\left(r_{\mathrm{Bo}}^{-\alpha_{S}+2}\right)+O\left(r_{\mathrm{Bo}}^{-\alpha_{r A}+2}\right)+O\left(r_{\mathrm{Bo}}^{-\alpha_{r r}+4}\right),
\end{aligned}
$$

and note that a factor $r_{\mathrm{Bo}}^{2}$ in the $O\left(r_{\mathrm{Bo}}^{-\alpha_{r r}+3}\right)$ terms in $\zeta^{\mathrm{Bo}}$ arises from the $4 \pi r_{\mathrm{Bo}} \bar{T}_{r r}^{\mathrm{Bo}} \zeta^{\mathrm{Bo}}$ term in (4.7), taking into account the $2 \Lambda r_{\mathrm{Bo}} / 3$ leading behavior of $\zeta^{\mathrm{Bo}}$.

We conclude that the leading order of all quantities of interest will be preserved if we assume that

$$
\alpha_{r r}>3, \alpha_{r A}>3, \alpha_{S}>3 .
$$

Keeping in mind our main assumptions, that all fields can be expanded in terms of inverse powers of $r_{\mathrm{Bo}}$ to the order needed to perform our expansions, possibly with some logarithmic coefficients, we will allow below matter fields for which (4.18) holds.

In what follows we will actually assume

$$
\bar{T}_{r r}^{\mathrm{Bo}}=O\left(r_{\mathrm{Bo}}^{-4}\right), \quad \bar{T}_{r A}^{\mathrm{Bo}}=O\left(r_{\mathrm{Bo}}^{-3}\right), \quad \bar{g}_{\mathrm{Bo}}^{A B} \bar{T}_{A B}^{\mathrm{Bo}}-\bar{T}^{\mathrm{Bo}}=O\left(r_{\mathrm{Bo}}^{-3}\right) .
$$

Note that the third equation in (4.19) is less restrictive than (4.18), allowing a logarithmic term in the asymptotic expansion of $\zeta^{\text {Bo }}$. This term however will be of the order $\log r_{\mathrm{Bo}} / r_{\mathrm{Bo}}^{2}$ and will not influence our result for the characteristic mass. It is accounted for in the correction term in (4.36) below. An analog statement holds for the fall-off behavior and the correction term in (5.16) below in affine coordinates.

When solving the wave-map gauge constraints we keep in mind that we eventually want to determine the expansion coefficient $\left(\bar{g}_{00}^{\mathrm{Bo}}\right)_{1}$, as needed to calculate the mass. This determines how far the intermediate asymptotic expansions need to be carried-out.

\subsection{Solving equation (3.11)}

The first equation of (3.23) implies $\tau^{\mathrm{Bo}}=2 r_{\mathrm{Bo}}^{-1}$ and using this one directly finds from (3.11) in Bondi-type coordinates, cf. (4.3),

$$
\kappa^{\mathrm{Bo}}=\frac{1}{2} r_{\mathrm{Bo}}\left(\left|\sigma^{\mathrm{Bo}}\right|^{2}+8 \pi \bar{T}_{r r}^{\mathrm{Bo}}\right) .
$$

Note for further reference that this means

$$
\left(\kappa^{\mathrm{Bo}}\right)_{n}=\frac{1}{2}\left[\left|\sigma^{\mathrm{Bo}}\right|_{n+1}^{2}+8 \pi\left(\bar{T}_{r r}^{\mathrm{Bo}}\right)_{n+1}\right]
$$

for the expansion coefficients of $\kappa^{\text {Bo }}$, where we have assumed that $n$ is positive. 


\subsection{Expansion of $\nu_{\mathrm{Bo}}^{0}$}

Inserting (3.24) and (4.20) into (3.12) in Bondi-type coordinates yields, cf. (4.4),

$$
\left[\partial_{r_{\mathrm{Bo}}}+\frac{r_{\mathrm{Bo}}}{2}\left(\left|\sigma^{\mathrm{Bo}}\right|^{2}+8 \pi \bar{T}_{r r}^{\mathrm{Bo}}\right)\right] \nu_{\mathrm{Bo}}^{0}=0,
$$

and from (4.1) we have

$$
\left|\sigma^{\mathrm{Bo}}\right|^{2}=\frac{\left|\sigma^{\mathrm{Bo}}\right|_{4}^{2}}{r_{\mathrm{Bo}}^{4}}+\frac{\left|\sigma^{\mathrm{Bo}}\right|_{5}^{2}}{r_{\mathrm{Bo}}^{5}}+\frac{\left|\sigma^{\mathrm{Bo}}\right|_{6}^{2}}{r_{\mathrm{Bo}}^{6}}+O\left(r_{\mathrm{Bo}}^{-7}\right) .
$$

Using this and (4.19) we find the solution of (4.22)

$$
\begin{aligned}
\nu_{\mathrm{Bo}}^{0}= & \left(\nu_{\mathrm{Bo}}^{0}\right)_{0}\left(1+\frac{1}{4}\left[\left|\sigma^{\mathrm{Bo}}\right|_{4}^{2}+8 \pi\left(\bar{T}_{r r}^{\mathrm{Bo}}\right)_{4}\right] r_{\mathrm{Bo}}^{-2}\right. \\
& \left.+\frac{1}{6}\left[\left|\sigma^{\mathrm{Bo}}\right|_{5}^{2}+8 \pi\left(\bar{T}_{r r}^{\mathrm{Bo}}\right)_{5}\right] r_{\mathrm{Bo}}^{-3}\right)+O\left(r_{\mathrm{Bo}}^{-4}\right),
\end{aligned}
$$

where $\left(\nu_{\mathrm{Bo}}^{0}\right)_{0}$ is a global integration function.

\subsection{Expansion of $\xi_{A}^{\text {Bo }}$}

Using $\partial_{A} \tau^{\text {Bo }}=0,(3.13)$ in Bondi-type coordinates takes the form

$$
\left(\partial_{r_{\mathrm{Bo}}}+\tau^{\mathrm{Bo}}\right) \xi_{A}^{\mathrm{Bo}}=2 \check{\nabla}_{B} \sigma_{A}^{\mathrm{Bo}}{ }_{A}^{B}-2 \partial_{A} \kappa^{\mathrm{Bo}}-16 \pi \bar{T}_{r A}^{\mathrm{Bo}},
$$

cf. (4.5). Using again (4.19), (4.23) as well as [28, Equations (3.24)-(3.26)] (as revisited to include matter fields)

$$
\check{\nabla}_{B} \sigma_{A}^{\mathrm{Bo}}{ }_{A}^{B}=\left(\Xi^{\mathrm{Bo}}\right)_{A}^{(2)} r_{\mathrm{Bo}}^{2}+\left(\Xi^{\mathrm{Bo}}\right)_{A}^{(3)} r_{\mathrm{Bo}}^{3}+O\left(r_{\mathrm{Bo}}^{-4}\right),
$$

where

$$
\left(\Xi^{\mathrm{Bo}}\right)_{A}^{(2)}:=\stackrel{\circ}{\nabla}_{B}\left(\sigma^{\mathrm{Bo}}{ }_{A}^{B}\right)_{2}, \quad\left(\Xi^{\mathrm{Bo}}\right)_{A}^{(3)}:=\stackrel{\circ}{\nabla}_{B}\left(\sigma^{\mathrm{Bo}}{ }_{A}^{B}\right)_{3}+\frac{1}{2} \stackrel{\circ}{\nabla}_{A}\left|\sigma^{\mathrm{Bo}}\right|_{4}^{2}+4 \pi \stackrel{\circ}{\nabla}_{A}\left(\bar{T}_{r r}^{\mathrm{Bo}}\right)_{4},
$$

the solution of $(4.25)$ reads

$$
\begin{aligned}
\xi_{A}^{\mathrm{Bo}}= & 2\left(\Xi^{\mathrm{Bo}}\right)_{A}^{(2)} r_{\mathrm{Bo}}^{-1}-2\left[\stackrel{\circ}{\nabla}_{B}\left(\sigma_{A}^{\mathrm{Bo}}{ }^{B}\right)_{3}-8 \pi\left(\bar{T}_{r A}^{\mathrm{Bo}}\right)_{3}\right] \frac{\log r_{\mathrm{Bo}}}{r_{\mathrm{Bo}}^{2}} \\
& +C_{A}^{\left(\xi_{B}\right)} r_{\mathrm{Bo}}^{-2}+O\left(r_{\mathrm{Bo}}^{-3}\right),
\end{aligned}
$$

where the coefficients $C_{A}^{\left(\xi_{B}\right)}=C_{A}^{\left(\xi_{B}\right)}\left(x^{C}\right)$ are global integration functions.

It follows from Proposition 2.1 that existence of a smooth conformal completion at infinity requires the relation

$$
\stackrel{\circ}{\nabla}_{B}\left(\sigma_{A}^{\mathrm{Bo}}{ }^{B}\right)_{3}=8 \pi\left(\bar{T}_{r A}^{\mathrm{Bo}}\right)_{3} .
$$




\subsection{Expansion of $\nu_{\mathrm{Bo}}^{A}$}

Equation (3.14) in Bondi-type coordinates does not depend upon $\Lambda$ and reads, cf. (4.6),

$$
\partial_{r_{\mathrm{Bo}}} \nu_{\mathrm{Bo}}^{A}=-\left(\check{\nabla}^{A}+\xi_{\mathrm{Bo}}^{A}\right) \nu_{0}^{\mathrm{Bo}} .
$$

Now, the transformation from the affine parameter $r$, described in Section 3.2 , to $r_{\text {Bo }}$ is given by (see [17, Equation (51) there])

$$
r_{\mathrm{Bo}}=r-\tau_{2} / 2+O\left(r^{-1}\right) .
$$

This implies that $\bar{g}_{\mathrm{Bo}}^{A B}$ is of the form

$$
\bar{g}_{\mathrm{Bo}}^{A B}=\stackrel{\circ}{h}^{A B} r_{\mathrm{Bo}}^{-2}+\left(\bar{g}_{\mathrm{Bo}}^{A B}\right)_{3} r_{\mathrm{Bo}}^{-3}+O\left(r_{\mathrm{Bo}}^{-4}\right),
$$

Using the form (4.24) of $\nu_{\mathrm{Bo}}^{0}$, keeping in mind the relation $\nu_{\mathrm{Bo}}^{0}=1 / \nu_{0}^{\mathrm{Bo}}$ and the form (4.28) of $\xi_{A}^{\mathrm{Bo}}$, we find the solution of (4.30)

$$
\begin{aligned}
\nu_{\mathrm{Bo}}^{A}= & \left(\nu_{\mathrm{Bo}}^{A}\right)_{0}+\stackrel{\circ}{h}^{A B} \check{\nabla}_{B}\left(\nu_{\mathrm{Bo}}^{0}\right)_{0}^{-1} r_{\mathrm{Bo}}^{-1} \\
& +\left[\left(\nu_{\mathrm{Bo}}^{0}\right)_{0}^{-1} \stackrel{\circ}{h}^{A B} \stackrel{\circ}{\nabla}_{C}\left(\sigma_{B}^{\mathrm{Bo} C}\right)_{2}+\frac{1}{2}\left(\bar{g}_{\mathrm{Bo}}^{A B}\right)_{3} \check{\nabla}_{B}\left(\nu_{\mathrm{Bo}}^{0}\right)_{0}^{-1}\right] r_{\mathrm{Bo}}^{-2} \\
& +\frac{2}{3} \stackrel{\circ}{h}^{A B}\left[\stackrel{\circ}{\nabla}_{A}\left(\sigma_{B}^{\mathrm{Bo} A}\right)_{3}-8 \pi\left(\bar{T}_{r B}^{\mathrm{Bo}}\right)_{3}\right] \frac{\log r_{\mathrm{Bo}}}{r_{\mathrm{Bo}}^{2}} \\
& -\frac{1}{3}\left[\frac{2}{3} \stackrel{\circ}{h}^{A B}\left(-\stackrel{\circ}{\nabla}_{A}\left(\sigma_{B}^{\mathrm{Bo} A}\right)_{3}+8 \pi\left(\bar{T}_{r B}^{\mathrm{Bo}}\right)_{3}\right)\right. \\
& +2\left(\bar{g}_{\mathrm{Bo}}^{A B}\right)_{3} \stackrel{\circ}{\nabla}_{A}\left(\sigma_{B}^{\mathrm{Bo} A}\right)_{2}+\stackrel{\circ}{h}^{A B} C_{B}^{\left(\xi_{C}\right)}-\left(\bar{g}_{\mathrm{Bo}}^{A B}\right)_{4} \stackrel{\circ}{\nabla}_{B}\left(\nu_{\mathrm{Bo}}^{0}\right)_{0}^{-1} \\
& \left.+\stackrel{\circ}{h}^{A B}\left(\nu_{\mathrm{Bo}}^{0}\right)_{2} \stackrel{\circ}{\nabla}_{B}\left(\nu_{\mathrm{Bo}}^{0}\right)_{0}^{-1}+\left(\nu_{\mathrm{Bo}}^{0}\right)_{0}^{-1} \stackrel{\circ}{h}^{A B} \stackrel{\circ}{\nabla}_{B}\left(\nu_{\mathrm{Bo}}^{0}\right)_{2}\right] r_{\mathrm{Bo}}^{-3} \\
& +\mathrm{o}\left(r_{\mathrm{Bo}}^{-3}\right),
\end{aligned}
$$

where $\left(\nu_{\mathrm{Bo}}^{A}\right)_{0}$ is a global integration function.

Note that the coefficient of the logarithmic term vanishes when (4.29) holds.

\subsection{Expansion of $\zeta^{\text {Bo }}$}

Inserting $\tau^{\text {Bo }}=2 r_{\text {Bo }}^{-1}$ and (4.20) into (3.15) in Bondi-type coordinates yields, cf. (4.7),

$$
\begin{aligned}
& \left(\partial_{r_{\mathrm{Bo}}}+\frac{2}{r_{\mathrm{Bo}}}+\frac{r_{\mathrm{Bo}}}{2}\left(\left|\sigma^{\mathrm{Bo}}\right|^{2}+8 \pi \bar{T}_{r r}^{\mathrm{Bo}}\right)\right) \zeta^{\mathrm{Bo}}= \\
& \quad-\check{R}^{\mathrm{Bo}}+\frac{\left|\xi^{\mathrm{Bo}}\right|^{2}}{2}-\check{\nabla}_{A} \xi_{\mathrm{Bo}}^{A}+8 \pi\left(\bar{g}_{\mathrm{Bo}}^{A B} \bar{T}_{A B}^{\mathrm{Bo}}-\bar{T}^{\mathrm{Bo}}\right)+2 \Lambda .
\end{aligned}
$$

In order to solve this equation we start by defining

$$
\zeta^{\mathrm{Bo}}:=\zeta_{\Lambda=0}^{\mathrm{Bo}}+\delta \zeta^{\mathrm{Bo}},
$$

where $\zeta_{\Lambda=0}^{\mathrm{Bo}}$ is the solution of (4.34) in the case $\Lambda=0$. Its asymptotic expansion is known: [28, Equation (3.40)] gives the formula in general coordinates for 
general $\stackrel{\circ}{R}$, while $[29$, Equation (5.23)] the one in Bondi-type coordinates with $\stackrel{\circ}{R}=2$ ) and reads

$$
\zeta_{\Lambda=0}^{\mathrm{Bo}}=-\frac{\stackrel{\circ}{R}}{r_{\mathrm{Bo}}}+\left(\zeta_{\Lambda=0}^{\mathrm{Bo}}\right)_{2} r_{\mathrm{Bo}}^{-2}+\mathrm{o}\left(r_{\mathrm{Bo}}^{-2}\right)
$$

where $\left(\zeta_{\Lambda=0}^{\text {Bo }}\right)_{2}$ is a global integration function and $\stackrel{\circ}{R}$ denotes the leading order coefficient of the asymptotic expansion of $\check{R}$ in terms of $r$, which coincides with the Ricci scalar of the boundary metric $\lim _{r \rightarrow \infty} r^{-2} \bar{g}_{A B} d x^{A} d x^{B}$. The expansion of $\delta \zeta^{\text {Bo }}$ on the other hand can be calculated by subtracting (4.34) from the corresponding equation in the case $\Lambda=0$, leading to

$$
\left(\partial_{r_{\mathrm{Bo}}}+\tau^{\mathrm{Bo}}+\kappa^{\mathrm{Bo}}\right) \delta \zeta^{\mathrm{Bo}}=2 \Lambda .
$$

This equation can be solved by using (4.19), (4.20) as well as (4.23) and we end up with

$$
\begin{aligned}
\delta \zeta^{\mathrm{Bo}}= & \frac{2 \Lambda}{3} r_{\mathrm{Bo}}-\frac{\Lambda}{3}\left[\left|\sigma^{\mathrm{Bo}}\right|_{4}^{2}+8 \pi\left(\bar{T}_{r r}^{\mathrm{Bo}}\right)_{4}\right] r_{\mathrm{Bo}}^{-1}+\frac{\Lambda}{3}\left[\left|\sigma^{\mathrm{Bo}}\right|_{5}^{2}+8 \pi\left(\bar{T}_{r r}^{\mathrm{Bo}}\right)_{5}\right] \frac{\log \left(r_{\mathrm{Bo}}\right)}{r_{\mathrm{Bo}}^{2}} \\
& +\frac{\left(\delta \zeta^{\mathrm{Bo}}\right)_{2}}{r_{\mathrm{Bo}}^{2}}+\mathrm{o}\left(r_{\mathrm{Bo}}^{-2}\right)
\end{aligned}
$$

where $\left(\delta \zeta^{\mathrm{Bo}}\right)_{2}$ is again a global integration function. Summing up the solution of (4.34) in Bondi-type coordinates reads

$$
\begin{aligned}
\zeta^{\mathrm{Bo}}= & \frac{2 \Lambda}{3} r_{\mathrm{Bo}}-\left(\stackrel{\circ}{R}+\frac{2 \Lambda}{3}\left(\kappa^{\mathrm{Bo}}\right)_{3}\right) r_{\mathrm{Bo}}^{-1} \\
& +\frac{2 \Lambda}{3}\left(\kappa^{\mathrm{Bo}}\right)_{4} \frac{\log r_{\mathrm{Bo}}}{r_{\mathrm{Bo}}^{2}}+\frac{\left(\zeta^{\mathrm{Bo}}\right)_{2}}{r_{\mathrm{Bo}}^{2}}+\mathrm{o}\left(r_{\mathrm{Bo}}^{-2}\right) \\
= & \frac{2 \Lambda}{3} r_{\mathrm{Bo}}-\left(\stackrel{\circ}{R}+\frac{\Lambda}{3}\left[\left|\sigma^{\mathrm{Bo}}\right|_{4}^{2}+8 \pi\left(\bar{T}_{r r}^{\mathrm{Bo}}\right)_{4}\right]\right) r_{\mathrm{Bo}}^{-1} \\
& +\frac{\Lambda}{3}\left[\left|\sigma^{\mathrm{Bo}}\right|_{5}^{2}+8 \pi\left(\bar{T}_{r r}^{\mathrm{Bo}}\right)_{5}\right] \frac{\log r_{\mathrm{Bo}}}{r_{\mathrm{Bo}}^{2}}+\frac{\left(\zeta^{\mathrm{Bo}}\right)_{2}}{r_{\mathrm{Bo}}^{2}}+\mathrm{o}\left(r_{\mathrm{Bo}}^{-2}\right),
\end{aligned}
$$

and we have combined both integration functions $\left(\delta \zeta^{\mathrm{Bo}}\right)_{2}$ and $\left(\zeta_{\Lambda=0}^{\mathrm{Bo}}\right)_{2}$ into $\left(\zeta^{\mathrm{Bo}}\right)_{2}$.

In view of the analysis of Section 2, existence of a smooth conformal completion leads to the condition

$$
\left(\kappa^{\mathrm{Bo}}\right)_{4}=0 \quad \Longleftrightarrow \quad\left|\sigma^{\mathrm{Bo}}\right|_{5}^{2}+8 \pi\left(\bar{T}_{r r}^{\mathrm{Bo}}\right)_{5}=0 .
$$

\subsection{Analyzing (3.16)}

Inserting (3.26) into (3.16) in Bondi-type coordinates and keeping in mind that, by (4.22), $\partial_{r_{\mathrm{Bo}}} \nu_{\text {Bo }}^{0}=-\kappa^{\mathrm{Bo}}$ one finds, cf. (4.8),

$$
\bar{g}_{\mathrm{Bo}}^{r r}+\left(\tau^{\mathrm{Bo}}\right)^{-1}\left(\zeta^{\mathrm{Bo}}-2 \nu_{\mathrm{Bo}}^{0} \check{\nabla}_{A} \nu_{\mathrm{Bo}}^{A}\right)=0
$$


for (3.16) in Bondi-type coordinates, which is an algebraic equation for $\bar{g}_{\mathrm{Bo}}^{r r}$. Inserting the asymptotic expansions (4.24), (4.33) and (4.39) we found for $\nu_{\mathrm{Bo}}^{0}$, $\nu_{\mathrm{Bo}}^{A}$ and $\zeta^{\mathrm{Bo}}$ respectively we obtain the asymptotic expansion

$$
\begin{aligned}
\bar{g}_{\mathrm{Bo}}^{r r}= & -\frac{\Lambda}{3} r_{\mathrm{Bo}}^{2}+\left(\nu_{\mathrm{Bo}}^{0}\right)_{0} \check{\nabla}_{A}\left(\nu_{\mathrm{Bo}}^{A}\right)_{0} r_{\mathrm{Bo}}+\left(\frac{\stackrel{\circ}{R}}{2}+\frac{\Lambda}{3}\left(\kappa^{\mathrm{Bo}}\right)_{3}\right)-\frac{\Lambda}{3}\left(\kappa^{\mathrm{Bo}}\right)_{4} \frac{\log r_{\mathrm{Bo}}}{r_{\mathrm{Bo}}} \\
& +\left(\left(\nu_{\mathrm{Bo}}^{0}\right)_{0}\left[\stackrel{\circ}{\nabla}_{A}\left(\nu_{\mathrm{Bo}}^{A}\right)_{2}+\left(\nu_{\mathrm{Bo}}^{0}\right)_{2} \stackrel{\circ}{\nabla}_{A}\left(\nu_{\mathrm{Bo}}^{A}\right)_{0}\right]-\frac{1}{2}\left(\zeta^{\mathrm{Bo}}\right)_{2}\right) r_{\mathrm{Bo}}^{-1} \\
& +\mathrm{o}\left(r_{\mathrm{Bo}}^{-1}\right)
\end{aligned}
$$

where, as before, $\stackrel{\circ}{R}$ is the Ricci scalar of the boundary metric $\lim _{r \rightarrow \infty} r^{-2} \bar{g}_{A B} d x^{A} d x^{B}$ and $\nabla_{A}$ is the associated covariant derivative.

Note that the coefficient of the logarithmic term in (4.39) vanishes if (4.40) holds.

\subsection{No-logs}

Consider characteristic initial data on $\mathscr{N}$ such that the functions $r_{\mathrm{Bo}}^{-2} g_{A B}^{\mathrm{Bo}}$ have a full asymptotic expansion in terms of inverse powers of $r_{\text {Bo }}$. From what has been said it follows that the equations

$$
\stackrel{\circ}{\nabla}_{B}\left(\sigma_{A}^{\mathrm{Bo}}{ }_{A}^{B}\right)_{3}-8 \pi\left(\bar{T}_{r A}^{\mathrm{Bo}}\right)_{3}=0=\left|\sigma^{\mathrm{Bo}}\right|_{5}^{2}+8 \pi\left(\bar{T}_{r r}^{\mathrm{Bo}}\right)_{5},
$$

(see (4.29) and (4.40)) provide a necessary condition for conformal smoothness of the associated space-time. It is likely that an analysis along the lines of [18], using $[22,23,30]$, will prove that these equations are also sufficient in spacetimes with conformally well-behaved matter fields (cf. [23-25]), but we have not investigated this.

When one, or both, of Equations (4.43) fails, the characteristic initial data set will have a full polyhomogeneous expansion at infinity. One expects that the evolved metric will similarly have a polyhomogeneous expansion, but no evolution theorems guaranteeing this are available so far even in vacuum.

As such, the no-logs conditions (4.43) require the data to be transformed to Bondi coordinates, if not already so given. When $\Lambda=0$, a coordinate-invariant version of the no-logs conditions has been established by Paetz in [28]. It would be of interest to find the equivalent of his conditions for $\Lambda \in \mathbb{R}^{*}$.

\section{Characteristic mass}

Throughout this section we assume that the space-time dimension is $n+1=4$.

\subsection{The Trautman-Bondi mass}

In $[5,31,35]$, assuming $\Lambda=0$ and in space-dimension $n=3$, it was proposed how to define the mass of a null hypersurface $\mathscr{N}$ at a given moment of "retarded 
time" $u$ at the cross-section $\stackrel{\mathscr{N}}{ }$ where it intersects null infinity $\mathscr{I}^{+}$. This mass, usually referred to as the Trautman-Bondi mass, is defined as

$$
m_{\mathrm{TB}}=\frac{1}{4 \pi} \int_{\mathscr{N}} M d \mu_{h}^{\circ}
$$

where ${ }^{3} d \mu_{h}=\sqrt{\operatorname{det} \stackrel{\circ}{h}_{A B}} d x^{2} d x^{3}$, and where $M$ denotes the mass aspect function $M: \stackrel{\mathscr{N}}{\rightarrow} \mathbb{R}$ (compare, e.g., $[5,31])$,

$$
M:=\frac{1}{2}\left(\bar{g}_{00}^{\mathrm{Bo}}\right)_{1} .
$$

The definition uses Bondi coordinates, as seen in Section 2.1, and recall that $\left(g_{00}^{\mathrm{Bo}}\right)_{k}$ denotes the coefficient in front of $1 / r_{\mathrm{Bo}}^{k}$ in an asymptotic expansion of $g_{00}^{\mathrm{Bo}}$ for large $r_{\mathrm{Bo}}$, in particular

$$
g_{00}^{\mathrm{Bo}}=-1+\frac{\left(g_{00}^{\mathrm{Bo}}\right)}{r_{\mathrm{Bo}}}+o\left(r_{\mathrm{Bo}}^{-1}\right) .
$$

Our aim is to obtain an analogue of the Trautman-Bondi mass in space-times with $\Lambda \neq 0$. We seek to derive a formula which applies to a class of space-times which includes vacuum space-times with a smooth conformal completion at null infinity $\mathscr{I}^{+}$, such that the characteristic surface intersects $\mathscr{I}^{+}$in a smooth cross-section $\stackrel{\mathscr{N}}{\text {. }}$

From our point of view, the key justification of (5.2) as providing a good candidate for the integrand for a total mass is the fact that $M$ is one of the nonlocal integration function which arise when solving the characteristic equations when $\Lambda=0$. It turns out that this remains true for $\Lambda \neq 0$.

Consider, thus, characteristic data in Bondi-type coordinates, defined perhaps only for large values of $r_{\text {Bo }}$. The space-time metric on $\mathscr{N}=\left\{u^{\mathrm{Bo}}=0\right\}$ can then be written as

$$
\bar{g}=\bar{g}_{00}^{\mathrm{Bo}} d u_{\mathrm{Bo}}^{2}+2 \nu_{0}^{\mathrm{Bo}} d u_{\mathrm{Bo}} d r_{\mathrm{Bo}}+2 \nu_{A}^{\mathrm{Bo}} d u_{\mathrm{Bo}} d x_{\mathrm{Bo}}^{A}+\check{g}^{\mathrm{Bo}} .
$$

Now, Bondi et al. assume

$$
\lim _{r_{\mathrm{Bo}} \rightarrow \infty} \nu_{\mathrm{Bo}}^{A}=0, \quad \lim _{r_{\mathrm{Bo}} \rightarrow \infty} \nu_{\mathrm{Bo}}^{0}=1, \quad \lim _{r_{\mathrm{Bo}} \rightarrow \infty}\left(r_{\mathrm{Bo}}^{-2} \bar{g}_{A B}^{\mathrm{Bo}}\right)=\stackrel{\circ}{h}_{A B} .
$$

It follows from Proposition 2.1 that the last equation in (5.4) is justified under the hypotheses there. However, it is not clear at all whether the first two can be assumed to hold for all retarded times in general: When $\Lambda<0$ this is part of asymptotic conditions which are usually imposed in this context, but which one might not want to impose in some situations. However, when $\Lambda>0$ there is little doubt that all three conditions in (5.4) can be simultaneously satisfied for all times by a restricted class of metrics only. For this reason we have allowed

\footnotetext{
${ }^{3}$ Bondi et al. introduced this formalism in the asymptotically flat case, where $\stackrel{\circ}{h}_{A B} \equiv s_{A B}$, the standard metric on $S^{2}$. In anticipation of other boundary topologies, e.g. a torus, we will use the symbol $h$ to denote the chosen metric on the relevant manifold.
} 
general fields $\left(\nu_{\mathrm{Bo}}^{A}\right)_{0}$ and $\left(\nu_{\mathrm{Bo}}^{0}\right)_{0}\left(x^{B}\right)$ when solving the constraint equations so far.

Nevertheless, it is easy to see that the first two equations (5.4) are determined by the propagation of the coordinates $u$ and $x^{A}$ away from the initial data surface $\mathscr{N}$, and can always be imposed on the $\mathscr{N}$ as long as one does not assume that they hold at later times. In particular, the first two equations in (5.4) imply no loss of generality as long as no evolution equations are used. Since we only work at $\mathscr{N}$, and use only the constraint equations, we will assume (5.4) from now on.

\subsection{The characteristic mass in Bondi-type coordinates}

The asymptotic expansion of $\bar{g}_{00}^{\text {Bo }}$ needed to obtain the mass aspect function can be calculated using the third equation in (3.7) in Bondi-type coordinates

$$
\bar{g}_{00}^{\mathrm{Bo}}=\bar{g}_{A B}^{\mathrm{Bo}} \nu_{\mathrm{Bo}}^{A} \nu_{\mathrm{Bo}}^{B}-\left(\nu_{0}^{\mathrm{Bo}}\right)^{2} \bar{g}_{\mathrm{Bo}}^{r r} .
$$

and we note again (cf. (4.32)) that $\bar{g}_{\mathrm{Bo}}^{A B}$ is of the form

$$
\bar{g}_{\mathrm{Bo}}^{A B}=\stackrel{\circ}{h}^{A B} r_{\mathrm{Bo}}^{-2}+\left(\bar{g}_{\mathrm{Bo}}^{A B}\right)_{3} r_{\mathrm{Bo}}^{-3}+O\left(r_{\mathrm{Bo}}^{-4}\right) .
$$

Using this and (4.42) leads us to

$$
\begin{aligned}
\bar{g}_{00}^{\mathrm{Bo}}= & \left(\stackrel{\circ}{h}_{A B}\left(\nu_{\mathrm{Bo}}^{A}\right)_{0}\left(\nu_{\mathrm{Bo}}^{B}\right)_{0}-\left(\nu_{\mathrm{Bo}}^{0}\right)_{0}^{-2}\left(\bar{g}_{\mathrm{Bo}}^{r r}\right)_{-2}\right) r_{\mathrm{Bo}}^{2} \\
& +\left(\bar{g}_{A B}^{\mathrm{Bo}}\right)_{-1}\left(\nu_{\mathrm{Bo}}^{A}\right)_{0}\left(\nu_{\mathrm{Bo}}^{B}\right)_{0} r_{\mathrm{Bo}} \\
& +\left(\nu_{\mathrm{Bo}}^{A}\right)_{0}\left[2 \stackrel{\circ}{h}_{A B}\left(\nu_{\mathrm{Bo}}^{B}\right)_{2}+\left(\bar{g}_{A B}^{\mathrm{Bo}}\right)_{0}\left(\nu_{\mathrm{Bo}}^{B}\right)_{0}\right] \\
& -\left(\nu_{\mathrm{Bo}}^{0}\right)_{0}^{-1}\left[\left(\nu_{\mathrm{Bo}}^{0}\right)_{0}^{-1}\left(\bar{g}_{\mathrm{Bo}}^{r r}\right)_{0}+2\left(\bar{g}_{\mathrm{Bo}}^{r r}\right)_{-2}\left(\nu_{0}^{\mathrm{Bo}}\right)_{2}\right] \\
& -\left(\nu_{\mathrm{Bo}}^{0}\right)_{0}^{-2}\left(\bar{g}_{\mathrm{Bo}}^{r r}\right)_{\log , 1} \frac{\log r_{\mathrm{Bo}}}{r_{\mathrm{Bo}}} \\
& +\left(\left(\nu_{\mathrm{Bo}}^{A}\right)_{0}\left[2 \stackrel{\circ}{h}_{A B}\left(\nu_{\mathrm{Bo}}^{B}\right)_{3}+2\left(\bar{g}_{A B}^{\mathrm{Bo}}\right)_{-1}\left(\nu_{\mathrm{Bo}}^{B}\right)_{2}+\left(\bar{g}_{A B}^{\mathrm{Bo}}\right)_{1}\left(\nu_{\mathrm{Bo}}^{B}\right)_{0}\right]\right. \\
& \left.-\left(\nu_{\mathrm{Bo}}^{0}\right)_{0}^{-1}\left[\left(\nu_{\mathrm{Bo}}^{0}\right)_{0}^{-1}\left(\bar{g}_{\mathrm{Bo}}^{r r}\right)_{1}+2\left(\bar{g}_{\mathrm{Bo}}^{r r}\right)_{-1}\left(\nu_{0}^{\mathrm{Bo}}\right)_{2}+2\left(\bar{g}_{\mathrm{Bo}}^{r r}\right)_{-2}\left(\nu_{0}^{\mathrm{Bo}}\right)_{3}\right]\right) r_{\mathrm{Bo}}^{-1} \\
& +\mathrm{o}\left(r_{\mathrm{Bo}}^{-1}\right),
\end{aligned}
$$

where we can directly read off an expression for the mass aspect function $M$ :

$$
\begin{aligned}
M= & \frac{1}{2}\left(\bar{g}_{00}^{\mathrm{Bo}}\right)_{1} \\
= & \frac{1}{2}\left(\left(\nu_{\mathrm{Bo}}^{A}\right)_{0}\left[2 \stackrel{\circ}{h}_{A B}\left(\nu_{\mathrm{Bo}}^{B}\right)_{3}+2\left(\bar{g}_{A B}^{\mathrm{Bo}}\right)_{-1}\left(\nu_{\mathrm{Bo}}^{B}\right)_{2}+\left(\bar{g}_{A B}^{\mathrm{Bo}}\right)_{1}\left(\nu_{\mathrm{Bo}}^{B}\right)_{0}\right]\right. \\
& \left.-\frac{\left(\nu_{\mathrm{Bo}}^{0}\right)_{0}^{-1}\left(\bar{g}_{\mathrm{Bo}}^{r r}\right)_{1}+2\left(\bar{g}_{\mathrm{Bo}}^{r r}\right)_{-1}\left(\nu_{0}^{\mathrm{Bo}}\right)_{2}+2\left(\bar{g}_{\mathrm{Bo}}^{r r}\right)_{-2}\left(\nu_{0}^{\mathrm{Bo}}\right)_{3}}{\left(\nu_{\mathrm{Bo}}^{0}\right)_{0}}\right) .
\end{aligned}
$$


Now, using that $\nu_{0}^{\mathrm{Bo}}=1 / \nu_{\mathrm{Bo}}^{0}(\mathrm{cf} .(3.7))$ and (4.24), we have

$$
\begin{aligned}
\nu_{0}^{\mathrm{Bo}}= & \left(\nu_{\mathrm{Bo}}^{0}\right)_{0}^{-1}\left(1-\left(\nu_{\mathrm{Bo}}^{0}\right)_{2} r_{\mathrm{Bo}}^{-2}-\left(\nu_{\mathrm{Bo}}^{0}\right)_{3} r_{\mathrm{Bo}}^{-3}\right)+O\left(r_{\mathrm{Bo}}^{-4}\right) \\
= & \left(\nu_{\mathrm{Bo}}^{0}\right)_{0}^{-1}\left(1-\frac{1}{4}\left[\left|\sigma^{\mathrm{Bo}}\right|_{4}^{2}+8 \pi\left(\bar{T}_{r r}^{\mathrm{Bo}}\right)_{4}\right] r_{\mathrm{Bo}}^{-2}\right. \\
& \left.-\frac{1}{6}\left[\left|\sigma^{\mathrm{Bo}}\right|_{5}^{2}+8 \pi\left(\bar{T}_{r r}^{\mathrm{Bo}}\right)_{5}\right] r_{\mathrm{Bo}}^{-3}\right)+O\left(r_{\mathrm{Bo}}^{-4}\right) .
\end{aligned}
$$

Inserting this and the expansion coefficients of $\bar{g}_{\mathrm{Bo}}^{r r}$ and $\nu_{\mathrm{Bo}}^{A}$ we calculated before $M$ reads

$$
\begin{aligned}
M= & \left(\nu_{\mathrm{Bo}}^{A}\right)_{0}\left[\stackrel{\circ}{h}_{A B}\left(\nu_{\mathrm{Bo}}^{B}\right)_{3}+\left(\bar{g}_{A B}^{\mathrm{Bo}}\right)_{-1}\left(\nu_{\mathrm{Bo}}^{B}\right)_{2}+\frac{1}{2}\left(\bar{g}_{A B}^{\mathrm{Bo}}\right)_{1}\left(\nu_{\mathrm{Bo}}^{B}\right)_{0}\right] \\
& +\left(\nu_{\mathrm{Bo}}^{0}\right)_{0}^{-1} \frac{1}{2}\left(\frac{1}{2}\left(\left(\zeta^{\mathrm{Bo}}\right)_{2}+\check{\nabla}_{A}\left(\nu_{\mathrm{Bo}}^{A}\right)_{0}\left[\left|\sigma^{\mathrm{Bo}}\right|_{4}^{2}+8 \pi\left(\bar{T}_{r r}^{\mathrm{Bo}}\right)_{4}\right]\right)\right. \\
& \left.-\dot{\nabla}_{A}\left(\nu_{\mathrm{Bo}}^{A}\right)_{2}-\left(\nu_{\mathrm{Bo}}^{0}\right)_{0}^{-1} \frac{\Lambda}{4}\left[\left|\sigma^{\mathrm{Bo}}\right|_{5}^{2}+8 \pi\left(\bar{T}_{r r}^{\mathrm{Bo}}\right)_{5}\right]\right) \\
& -\frac{1}{8}\left[\left|\sigma^{\mathrm{Bo}}\right|_{4}^{2}+8 \pi\left(\bar{T}_{r r}^{\mathrm{Bo}}\right)_{4}\right] \stackrel{\circ}{\nabla}_{A}\left(\nu_{\mathrm{Bo}}^{A}\right)_{0} .
\end{aligned}
$$

We return, now, to the definition of the characteristic mass, (5.1), and assume in the remainder of the present work that the boundary conditions on

$\nu_{\mathrm{Bo}}^{0}$ and $\nu_{\mathrm{Bo}}^{A}$, introduced in (5.4), hold. With these boundary conditions, and using the fact that the divergence terms in (5.10) will integrate out to zero, we find

$$
\begin{aligned}
m_{\mathrm{TB}} & =\frac{1}{16 \pi} \int_{\mathscr{\mathscr { N }}}\left(\zeta^{\mathrm{Bo}}\right)_{2} d \mu_{h}+\frac{\Lambda}{12 \pi} \int_{\mathscr{\mathscr { N }}}\left(\nu_{0}^{\mathrm{Bo}}\right)_{3} d \mu_{h} \\
& =\frac{1}{16 \pi} \int_{\mathscr{N}}\left(\zeta^{\mathrm{Bo}}\right)_{2} d \mu_{h}-\frac{\Lambda}{72 \pi} \int_{\mathscr{N}}\left[\left|\sigma^{\mathrm{Bo}}\right|_{5}^{2}+8 \pi\left(\bar{T}_{r r}^{\mathrm{Bo}}\right)_{5}\right] d \mu_{h} .
\end{aligned}
$$

\subsection{The characteristic mass in terms of characteristic data}

To continue, we want to relate the fields occurring in Bondi-type coordinates to their representation in coordinates where $r$ is an affine parameter along the radial null outgoing geodesics of $\bar{g}$. We start with $\left(\zeta^{\mathrm{Bo}}\right)_{2}$ and follow the argumentation in [17, leading to Equation (51) there], which we repeat here for the convenience of the reader.

First, we have

$$
r_{\mathrm{Bo}}=r-\frac{\tau_{2}}{2}+O\left(r^{-1}\right) .
$$

Next, the transformation formulae for $\tau$ and $\zeta$ (compare (3.19)) read:

$$
\begin{aligned}
\tau^{\mathrm{Bo}}\left(r^{\mathrm{Bo}}\right) & =\frac{\partial r}{\partial r^{\mathrm{Bo}}} \tau\left(r\left(r^{\mathrm{Bo}}\right)\right)=\frac{2}{r_{\mathrm{Bo}}} \\
\zeta^{\mathrm{Bo}} & =2\left(\bar{g}^{\mathrm{Bo}}\right)^{A B}\left(\bar{\Gamma}^{\mathrm{Bo}}\right)_{A B}^{r_{\mathrm{Bo}}}+\tau^{\mathrm{Bo}}\left(\bar{g}^{\mathrm{Bo}}\right)^{r_{\mathrm{Bo}} r_{\mathrm{Bo}}} \\
& =2\left(\bar{g}^{\mathrm{Bo}}\right)^{A B}\left(\frac{\partial r_{\mathrm{Bo}}}{\partial x^{k}} \frac{\partial x^{i}}{\partial x_{\mathrm{Bo}}^{A}} \frac{\partial x^{j}}{\partial x_{\mathrm{Bo}}^{B}} \bar{\Gamma}_{i j}^{k}+\frac{\partial r_{\mathrm{Bo}}}{\partial r} \frac{\partial^{2} r}{\partial x_{\mathrm{Bo}}^{A} \partial x_{\mathrm{Bo}}^{B}}\right)
\end{aligned}
$$




$$
\begin{aligned}
+ & +\frac{\partial r}{\partial r_{\mathrm{Bo}}} \frac{\partial r_{\mathrm{Bo}}}{\partial x^{i}} \frac{\partial r_{\mathrm{Bo}}}{\partial x^{j}} \bar{g}^{i j} \\
= & 2 \bar{g}^{A B} \frac{\partial r_{\mathrm{Bo}}}{\partial r} \frac{\partial r}{\partial x_{\mathrm{Bo}}^{A}} \frac{\partial r}{\partial x_{\mathrm{Bo}}^{B}} \kappa+2 \bar{g}^{A B} \frac{\partial r_{\mathrm{Bo}}}{\partial x^{C}} \frac{\partial r}{\partial x_{\mathrm{Bo}}^{A}} \frac{\partial r}{\partial x_{\mathrm{Bo}}^{B}} \underbrace{\bar{\Gamma}_{11}^{C}}_{=0} \\
& +\frac{\partial r_{\mathrm{Bo}}}{\partial r} \zeta+2 \bar{g}^{A B} \frac{\partial r_{\mathrm{Bo}}}{\partial x^{C}} \tilde{\Gamma}_{A B}^{C}+2 \underbrace{\bar{g}^{A B} \chi_{A B}}_{=\tau} \frac{\partial r_{\mathrm{Bo}}}{\partial x^{C}} \nu^{0} \nu^{C} \\
& -2 \bar{g}^{A B} \frac{\partial r_{\mathrm{Bo}}}{\partial r} \frac{\partial r}{\partial x_{\mathrm{Bo}}^{B}} \xi_{A}+2 \tau \bar{g}^{A B} \frac{\partial r_{\mathrm{Bo}}}{\partial x^{A}} \frac{\partial r}{\partial x_{\mathrm{Bo}}^{B}}+4 \bar{g}^{A B} \frac{\partial r_{\mathrm{Bo}}}{\partial x^{C}} \frac{\partial r}{\partial x_{\mathrm{Bo}}^{B}} \sigma_{A}^{C} \\
& +2 \bar{g}^{A B} \frac{\partial r_{\mathrm{Bo}}}{\partial r} \frac{\partial^{2} r}{\partial x_{\mathrm{Bo}}^{A} \partial x_{\mathrm{Bo}}^{B}}+\tau \frac{\partial r}{\partial r_{\mathrm{Bo}}} \frac{\partial r_{\mathrm{Bo}}}{\partial x^{A}} \frac{\partial r_{\mathrm{Bo}}}{\partial x^{B}} \bar{g}^{A B} \\
= & \frac{\partial r_{\mathrm{Bo}}}{\partial r} \zeta+2 \frac{\partial r_{\mathrm{Bo}}}{\partial r} \Delta_{\check{g}} r+O\left(r_{\mathrm{Bo}}^{-3}\right),
\end{aligned}
$$

where $\Delta_{\check{g}}$ is the Laplace operator of the two-dimensional metric $\check{g}_{A B} d x_{\mathrm{Bo}}^{A} d x_{\mathrm{Bo}}^{B}$.

To continue we need the asymptotic expansion of $\zeta$ and therefore solve the respective constraint equation (3.15). Note that we have already done this in Bondi-type coordinates, but we also need the result in affine coordinates.

We begin with the same procedure as in Section 4.6 and define

$$
\zeta:=\zeta_{\Lambda=0}+\delta \zeta
$$

where $\zeta_{\Lambda=0}$ is the solution of (3.15) in the case $\Lambda=0$. Its asymptotic expansion is known and reads [28, Equation (3.40)]

$$
\zeta_{\Lambda=0}=-\frac{\stackrel{\circ}{R}}{r}+\left(\zeta_{\Lambda=0}\right)_{2} r^{-2}+\mathrm{o}\left(r^{-2}\right)
$$

with $\left(\zeta_{\Lambda=0}\right)_{2}$ being a global integration function. We assume that the relevant fields satisfy analog fall-off behavior in affine coordinate $r$ as we assumed in Bondi-type coordinate $r_{\text {Bo }}$ (cf. Equations (4.1) and (4.19)). The equation for $\delta \zeta$ reads

$$
\left(\partial_{r}+\tau+\kappa\right) \delta \zeta=2 \Lambda .
$$

From now on we choose the coordinate $r$ so that $\kappa=0$. We start by solving the Raychaudhuri equation (3.11) in this gauge and obtain the expansion of $\tau$

$$
\begin{aligned}
\tau= & \frac{2}{r}+\frac{\tau_{2}}{r^{2}}+\frac{2\left[|\sigma|_{4}^{2}+8 \pi\left(\bar{T}_{r r}\right)_{4}\right]+\tau_{2}^{2}}{2 r^{3}} \\
& +\frac{2\left[|\sigma|_{5}^{2}+8 \pi\left(\bar{T}_{r r}\right)_{5}\right]+2 \tau_{2}\left[|\sigma|_{4}^{2}+8 \pi\left(\bar{T}_{r r}\right)_{4}\right]+\tau_{2}^{3}}{4 r^{4}}+O\left(r^{-5}\right)
\end{aligned}
$$

where $\tau_{2}$ is a global integration function and $|\sigma|_{n}^{2}$ are the expansion coefficients of $|\sigma|^{2}$ :

$$
|\sigma|^{2}=\frac{|\sigma|_{4}^{2}}{r^{4}}+\frac{|\sigma|_{5}^{2}}{r^{5}}+O\left(r^{-6}\right)
$$


Using (5.18) we find from (5.17)

$$
\begin{aligned}
\delta \zeta= & \Lambda\left(\frac{2 r}{3}-\frac{\tau_{2}}{3}+\frac{\tau_{2}^{2}-2 \tau_{3}}{3 r}+\frac{3 \tau_{2} \tau_{3}-\tau_{2}^{3}-2 \tau_{4}}{3} \frac{\log r}{r^{2}}\right) \\
& +\frac{\delta \zeta_{2}}{r^{2}}+\mathrm{o}\left(r^{-2}\right),
\end{aligned}
$$

where $\delta \zeta_{2}$ is again a global integration function. Summing, and combining the two integration functions $\left(\zeta_{\Lambda=0}\right)_{2}$ and $\delta \zeta_{2}$ into $\zeta_{2}$ the solution of (3.15) gives

$$
\begin{aligned}
\zeta= & \frac{2 \Lambda}{3} r-\frac{\Lambda \tau_{2}}{3}-\left(\stackrel{\circ}{R}+\frac{\Lambda\left(2 \tau_{3}-\tau_{2}^{2}\right)}{3}\right) r^{-1} \\
& +\frac{\Lambda\left(3 \tau_{2} \tau_{3}-\tau_{2}^{3}-2 \tau_{4}\right)}{3} \frac{\log r}{r^{2}}+\frac{\zeta_{2}}{r^{2}}+\mathrm{o}\left(r^{-2}\right) .
\end{aligned}
$$

Using this and the asymptotic expansion of $\Delta_{\breve{g}} r$ (compare [17, Equation (51)])

$$
\Delta_{\check{g}} r=\frac{\Delta_{\grave{h}} \tau_{2}}{2 r^{2}}+O\left(r^{-3}\right)
$$

and expressing (5.14) in terms of $r_{\text {Bo }}$ one obtains

$$
\begin{aligned}
\left(\zeta^{\mathrm{Bo}}\right)_{2}= & \zeta_{2}+\frac{\stackrel{\circ}{2} \tau_{2}+\Delta_{h} \tau_{2}}{} \\
& +\frac{\Lambda}{3}\left(-\frac{\left|\sigma^{\mathrm{Bo}}\right|_{5}^{2}+8 \pi\left(\bar{T}_{r r}^{\mathrm{Bo}}\right)_{5}}{3}+\tau_{2}\left[|\sigma|_{4}^{2}+8 \pi\left(\bar{T}_{r r}\right)_{4}\right]\right) \\
\zeta_{\log , 2}^{\mathrm{Bo}}= & \frac{\Lambda}{3}\left(2 \tau_{2}\left[|\sigma|_{4}^{2}+8 \pi\left(\bar{T}_{r r}\right)_{4}\right]-|\sigma|_{5}^{2}-8 \pi\left(\bar{T}_{r r}\right)_{5}\right)
\end{aligned}
$$

Inserting (5.23) into (5.10) and using the boundary conditions on $\nu_{\mathrm{Bo}}^{0}$ and $\nu_{\mathrm{Bo}}^{A}$, introduced in (5.4), we find

$$
\begin{aligned}
M= & \frac{1}{4}\left(\zeta_{2}+\frac{\stackrel{\circ}{R}}{2} \tau_{2}+\Delta_{h} \tau_{2}\right)-\frac{1}{2} \stackrel{\circ}{\nabla}^{A}\left(\nu_{A}^{\mathrm{Bo}}\right)_{0} \\
& -\frac{\Lambda}{12}\left(\left|\sigma^{\mathrm{Bo}}\right|_{5}^{2}+8 \pi\left(\bar{T}_{r r}^{\mathrm{Bo}}\right)_{5}-\tau_{2}\left[|\sigma|_{4}^{2}+8 \pi\left(\bar{T}_{r r}\right)_{4}\right]\right) .
\end{aligned}
$$

We now calculate the expansions of $|\sigma|^{2}$ and $\left|\sigma^{\text {Bo }}\right|^{2}$ to insert explicit expressions for the coefficients occurring in $M$. Further we want to relate the relevant coefficients of the energy-momentum tensor in Bondi-type coordinates to their representation in affine coordinates. For $|\sigma|^{2}$ one obviously has

$$
\begin{aligned}
|\sigma|^{2} & =\frac{|\sigma|_{4}^{2}}{r^{4}}+\frac{|\sigma|_{5}^{2}}{r^{5}}+O\left(r^{-6}\right) \\
& =\frac{\left(\sigma_{A}^{B}\right)_{2}\left(\sigma_{B}^{A}\right)_{2}}{r^{4}}+2 \frac{\left(\sigma_{A}^{B}\right)_{2}\left(\sigma_{B}^{A}\right)_{3}}{r^{5}}+O\left(r^{-6}\right)
\end{aligned}
$$


and performing a coordinate transformation and replacing the dependence on $r$ with $r_{\text {Bo }}$ we obtain

$$
\begin{aligned}
\left|\sigma^{\mathrm{Bo}}\right|^{2}= & \frac{\left(\sigma_{A}^{B}\right)_{2}\left(\sigma_{B}^{A}\right)_{2}}{r_{\text {Bo }}^{4}}+2 \frac{\left(\sigma_{A}^{B}\right)_{2}\left(\sigma_{B}{ }^{A}\right)_{3}-\left(\sigma_{A}^{B}\right)_{2}\left(\sigma_{B}{ }^{A}\right)_{2} \tau_{2}}{r_{\text {Bo }}^{5}} \\
& +O\left(r_{\text {Bo }}^{-6}\right) \\
= & \frac{|\sigma|_{4}^{2}}{r_{\text {Bo }}^{4}}+\frac{|\sigma|_{5}^{2}-2|\sigma|_{4}^{2} \tau_{2}}{r_{\text {Bo }}^{5}}+O\left(r_{\text {Bo }}^{-6}\right) .
\end{aligned}
$$

By assumption, or by smooth conformal compactifiability we can write $\bar{T}_{r r}^{\text {Bo }}$ in the form

$$
\bar{T}_{r r}^{\mathrm{Bo}}=\frac{\left(\bar{T}_{r r}^{\mathrm{Bo}}\right)_{4}}{r_{\mathrm{Bo}}^{4}}+\frac{\left(\bar{T}_{r r}^{\mathrm{Bo}}\right)_{5}}{r_{\mathrm{Bo}}^{5}}+O\left(r_{\mathrm{Bo}}^{-6}\right) .
$$

Performing a coordinate transformation we find an analog expansion for $\bar{T}_{r r}$ in affine coordinates and replacing again the dependence on $r$ with $r_{\text {Bo }}$ we obtain

$$
\bar{T}_{r r}^{\mathrm{Bo}}=\frac{\left(\bar{T}_{r r}\right)_{4}}{r_{\mathrm{Bo}}^{4}}+\frac{\left(\bar{T}_{r r}\right)_{5}-2\left(\bar{T}_{r r}\right)_{4} \tau_{2}}{r_{\mathrm{Bo}}^{5}}+O\left(r_{\mathrm{Bo}}^{-6}\right) .
$$

Therefore we end up with the following formula for the mass aspect expressed through characteristic data

$$
\begin{aligned}
M= & \frac{1}{4}\left(\zeta_{2}+\frac{\stackrel{\circ}{R}}{2} \tau_{2}+\Delta_{h} \tau_{2}\right)-\frac{1}{2} \stackrel{\circ}{\nabla^{A}}\left(\nu_{A}^{\mathrm{Bo}}\right)_{0} \\
& +\frac{\Lambda}{12}\left(3 \tau_{2}\left[|\sigma|_{4}^{2}+8 \pi\left(\bar{T}_{r r}\right)_{4}\right]-\left[|\sigma|_{5}^{2}+8 \pi\left(\bar{T}_{r r}\right)_{5}\right]\right) .
\end{aligned}
$$

Using again the definition of the characteristic mass and bearing in mind that the divergence terms will vanish after integration over $\mathscr{N}$ we find

$$
\begin{aligned}
m_{\mathrm{TB}}= & \frac{1}{16 \pi} \int_{\mathscr{N}}\left(\zeta_{2}+\frac{\stackrel{\circ}{R}}{2} \tau_{2}\right) d \mu_{h} \\
& +\frac{\Lambda}{48 \pi} \int_{\mathscr{N}}\left(3 \tau_{2}\left[|\sigma|_{4}^{2}+8 \pi\left(\bar{T}_{r r}\right)_{4}\right]-\left[|\sigma|_{5}^{2}+8 \pi\left(\bar{T}_{r r}\right)_{5}\right]\right) d \mu_{h}
\end{aligned}
$$

\subsection{The characteristic mass and the renormalized volume}

We are ready to prove our final formula for the characteristic mass, which will be in terms of geometric fields defined on a characteristic surface parametrized by an affine parameter $r$ ranging from $r_{0}$ to infinity. In the case of a light-cone we take $r_{0}=0$, but we allow non-zero $r_{0}$ to cover other situations of interest.

We first note the asymptotic expansion of $\sqrt{\operatorname{det} \bar{g}_{A B}}$ for large $r$, which is obtained by using the considerations in [28, leading to equation (3.13) there] 
and our result for the expansion of $\tau,(5.18)$ :

$$
\begin{aligned}
\sqrt{\operatorname{det} \bar{g}_{A B}} & =r^{2} \sqrt{\operatorname{det} \stackrel{\circ}{h}_{A B}}\left(1-\frac{\tau_{2}}{r}+\frac{\tau_{2}^{2}-2\left[|\sigma|_{4}^{2}+8 \pi\left(\bar{T}_{r r}\right)_{4}\right]}{4 r^{2}}\right. \\
& \left.+\frac{2 \tau_{2}\left[|\sigma|_{4}^{2}+8 \pi\left(\bar{T}_{r r}\right)_{4}\right]-\left[|\sigma|_{5}^{2}+8 \pi\left(\bar{T}_{r r}\right)_{5}\right]}{6 r^{3}}+O\left(r^{-4}\right)\right)
\end{aligned}
$$

Using this, $d \mu_{\check{g}}=\sqrt{\operatorname{det} \bar{g}_{A B}} d x^{2} d x^{3}$ and the expansion (5.21) of $\zeta$ we find

$$
\begin{aligned}
& \int_{\mathscr{N}} \zeta d \mu_{\check{g}}=\frac{2 \Lambda}{3} r^{3} \underbrace{\int_{\mathscr{\mathscr { N }}} d \mu_{\grave{h}}}_{=: \mu_{\grave{h}}(\mathscr{\mathscr { N }})}-\Lambda r^{2} \int_{\mathscr{N}} \tau_{2} d \mu_{\grave{h}}-r \int_{\mathscr{\mathscr { N }}} \stackrel{R}{R} d \mu_{h} \\
& -\Lambda r \int_{\mathscr{N}}\left(\left[|\sigma|_{4}^{2}+8 \pi\left(\bar{T}_{r r}\right)_{4}\right]-\frac{1}{2} \tau_{2}^{2}\right) d \mu_{h} \\
& -\frac{\Lambda}{3} \log r \int_{\mathscr{\mathscr { N }}}\left(\left[|\sigma|_{5}^{2}+8 \pi\left(\bar{T}_{r r}\right)_{5}\right]-2\left[|\sigma|_{4}^{2}+8 \pi\left(\bar{T}_{r r}\right)_{4}\right] \tau_{2}\right) d \mu_{h} \\
& +\int_{\mathscr{N}}\left[\zeta_{2}+\stackrel{\circ}{R} \tau_{2}\right] d \mu_{h}-\frac{\Lambda}{12} \int_{\mathscr{N}} \tau_{2}^{3} d \mu_{h} \\
& +\frac{\Lambda}{18} \int_{\mathscr{N}}\left(19 \tau_{2}\left[|\sigma|_{4}^{2}+8 \pi\left(\bar{T}_{r r}\right)_{4}\right]-2\left[|\sigma|_{5}^{2}+8 \pi\left(\bar{T}_{r r}\right)_{5}\right]\right) d \mu_{h}+\mathrm{o}(1) \text {. }
\end{aligned}
$$

From (3.15) with $\kappa=0$ and the Gauss-Bonnet theorem we have,

$$
\int_{\mathscr{N}}\left(\partial_{r}+\tau\right) \zeta d \mu_{\check{g}}=-4 \pi \chi(\stackrel{\mathscr{N}}{)})+\int_{\mathscr{N}}\left(\frac{1}{2}|\xi|^{2}+S\right) d \mu_{\check{g}}+2 \Lambda \int_{\mathscr{N}} d \mu_{\check{g}}
$$

where $\chi(\stackrel{\mathscr{N}}{)})$ is the Euler characteristic of $\mathscr{N}$. The integral in the last term of that equation is the area of the constant- $r$ sections of $\mathscr{N}$, and we define the volume function $V(r)$ to be its integral

$$
V(r):=\int_{\tilde{r}=r_{0}}^{r} \frac{d V(\tilde{r})}{d \tilde{r}} d \tilde{r}=\int_{\tilde{r}=r_{0}}^{r} \int_{\mathscr{S}} d \mu_{\tilde{g}} d \tilde{r} .
$$

REMARK 5.1 We note that $V(r)$ is uniquely defined up to the choice $r_{0}$ of the origin of $r$ and up to scaling on each generator.

When cross-sections of $\mathscr{I}$ are negatively curved compact manifolds, the asymptotic conditions imposed in our construction define the scaling uniquely.

When cross-sections of $\mathscr{I}$ are flat compact manifolds, the asymptotic conditions imposed in our construction define the scaling up to a constant. This freedom can be gotten rid of by requiring the $h$-volume of the cross-section to take some convenient value, e.g. one or $(2 \pi)^{2}$.

When cross-sections of $\mathscr{I}$ are two-dimensional spheres, the asymptotic conditions imposed in our construction define the scaling uniquely up to the action of the group of conformal transformations of $S^{2}$. This freedom reflects the fact that in this case $m_{\mathrm{TB}}$ is not a mass but the time-component of a covector. 
A redefinition of $r_{0}$ affects the explicit formula for $V$ as a function of $r$, and hence the numerical value of the "renormalized volume", to be defined shortly. When $\mathscr{N}$ is a globally smooth light-cone, or is a smooth hypersurface emitted from a submanifold of codimension larger than one, then the origin of the affine parameter $r_{0}=0$ is determined by the location of the "emitting" submanifold, which gets rid of the last ambiguity.

Using $\partial_{r} \sqrt{\operatorname{det} \bar{g}_{A B}}=\tau \sqrt{\operatorname{det} \bar{g}_{A B}}$ we find

$$
\partial_{r} \int_{\mathscr{\mathscr { N }}} \zeta d \mu_{\check{g}}=-4 \pi \chi(\stackrel{\circ}{\mathscr{N}})+2 \Lambda \frac{d V(r)}{d r}+\int_{\mathscr{N}}\left(\frac{1}{2}|\xi|^{2}+S\right) d \mu_{\check{g}}
$$

which we can integrate in $r$ starting from $r=r_{0}$

$$
\begin{aligned}
\lim _{r \rightarrow \infty} & \left(\int_{\mathscr{N}} \zeta d \mu_{\breve{g}}+4 \pi \chi(\stackrel{\mathscr{N}}{)}) r-2 \Lambda V(r)\right) \\
\quad & =\lim _{r \rightarrow r_{0}} \int_{\mathscr{\mathscr { N }}} \zeta d \mu_{\check{g}}+4 \pi \chi(\stackrel{\mathscr{N}}{)}) r_{0}+\int_{r=r_{0}}^{\infty} \int_{\mathscr{N}}\left(\frac{1}{2}|\xi|^{2}+S\right) d \mu_{\check{g}} d r .
\end{aligned}
$$

We leave the symbol $\lim _{r \rightarrow r_{0}}$ in the last equation to accommodate a vertex at $r=r_{0}$, where $\zeta$ is singular, but note that light-surfaces emanating from smooth space co-dimension-two submanifolds will also be of interest to us. One needs to make sure to use appropriate boundary conditions for the lower bound of the integration depending on what kind of characteristic surface is studied. In the case of a light-cone, i.e. a null-hypersurface emanating from a point at $r_{0}=0$, the necessary boundary conditions follow from regularity at the tip of the cone as has been discussed in [7, Section 4.5].

When the first term in the last line vanishes, we can infer non-negativity of the left-hand side by assuming the dominant energy condition for non-vanishing matter fields. This condition implies then [29]

$$
S:=8 \pi\left(\bar{g}^{A B} \bar{T}_{A B}-\bar{T}\right) \geq 0
$$

which means that the right-hand side of (5.37) is manifestly non-negative. Assuming that the right-hand side of (5.37) is finite, we see that the divergent terms in $2 \Lambda V(r)$ and $4 \pi \chi(\mathscr{\mathscr { N }}) r$ need to cancel those in the expression on the right-hand side of (5.33) exactly. To make this precise we continue by calculating an explicit expression for the volume function $V(r)$. We start by using again (5.32) and find

$$
\begin{aligned}
\frac{d V(r)}{d r}= & \int_{\mathscr{\mathscr { N }}} d \mu_{\check{g}}=r^{2} \mu_{\grave{h}}(\mathscr{N})-r \int_{\mathscr{N}} \tau_{2} d \mu_{\grave{h}} \\
& +\frac{1}{2} \int_{\mathscr{\mathscr { N }}}\left(\frac{1}{2} \tau_{2}^{2}-\left[|\sigma|_{4}^{2}+8 \pi\left(\bar{T}_{r r}\right)_{4}\right]\right) d \mu_{\grave{h}} \\
& +\frac{1}{6 r} \int_{\mathscr{\mathscr { V }}}\left(2\left[|\sigma|_{4}^{2}+8 \pi\left(\bar{T}_{r r}\right)_{4}\right] \tau_{2}-\left[|\sigma|_{5}^{2}+8 \pi\left(\bar{T}_{r r}\right)_{5}\right]\right) d \mu_{h} \\
& +O\left(r^{-2}\right) .
\end{aligned}
$$


It follows that there exist constants so that the function $V(r)$ has an asymptotic expansion of the form

$$
V(r)=\frac{1}{3} r^{3} \mu_{h}(\stackrel{\circ}{\mathscr{N}})+V_{-2} r^{2}+V_{-1} r+V_{\log } \log r+V_{0}+V_{1} r^{-1}+o\left(r^{-1}\right) .
$$

We define the renormalized volume $V_{\text {ren }}$ as "the finite left-over in the expansion":

$$
V_{\text {ren }}:=V_{0} \text {. }
$$

One can think of $V_{\text {ren }}$ as the global integration function arising from integrating the equation for $d V / d r$. The numerical value of $V_{\text {ren }}$ is defined up to the ambiguities pointed out in Remark 5.1.

Integrating (5.39) we obtain in fact

$$
\begin{aligned}
-2 \Lambda V(r)= & \Lambda\left[-\frac{2}{3}\left(\left(r-\frac{\tau_{2}}{2}\right)^{3}+\left(\frac{\tau_{2}}{2}\right)^{3}\right) \mu_{\grave{h}}(\stackrel{\circ}{\mathscr{N}})-2 V_{\text {ren }}\right. \\
& +r \int_{\mathscr{N}}\left(\left[|\sigma|_{4}^{2}+8 \pi\left(\bar{T}_{r r}\right)_{4}\right]\right) d \mu_{\grave{h}} \\
& \left.+\frac{1}{3} \log r \int_{\mathscr{N}}\left(\left[|\sigma|_{5}^{2}+8 \pi\left(\bar{T}_{r r}\right)_{5}\right]-2\left[|\sigma|_{4}^{2}+8 \pi\left(\bar{T}_{r r}\right)_{4}\right] \tau_{2}\right) d \mu_{h}\right] \\
& +O\left(r^{-1}\right),
\end{aligned}
$$

thus

$$
\begin{aligned}
V_{\text {ren }} & =\lim _{r \rightarrow \infty}\left[V(r)-\frac{r^{3}}{3} \mu_{h}(\stackrel{\mathscr{N}}{)})+\frac{r^{2}}{2} \int_{\mathscr{N}} \tau_{2} d \mu_{h}\right. \\
& +\frac{r}{2} \int_{\mathscr{N}}\left(\left[|\sigma|_{4}^{2}+8 \pi\left(\bar{T}_{r r}\right)_{4}\right]-\frac{1}{2} \tau_{2}^{2}\right) d \mu_{h}^{\circ} \\
& \left.+\frac{1}{6} \log r \int_{\mathscr{N}}\left(\left[|\sigma|_{5}^{2}+8 \pi\left(\bar{T}_{r r}\right)_{5}\right]-2\left[|\sigma|_{4}^{2}+8 \pi\left(\bar{T}_{r r}\right)_{4}\right] \tau_{2}\right) d \mu_{h}\right] .
\end{aligned}
$$

Now, by (5.37) and using (5.33) and (5.40),

$$
\begin{aligned}
& \lim _{r \rightarrow \infty}\left(\int_{\mathscr{N}} \zeta d \mu_{\check{g}}+4 \pi \chi(\stackrel{\circ}{\mathscr{N}}) r-2 \Lambda V(r)\right) \\
& =\lim _{r \rightarrow \infty}\left(-r \int_{\mathscr{N}} \stackrel{\circ}{R} d \mu_{h}+4 \pi \chi(\stackrel{\mathscr{N}}{)} r)-\frac{\Lambda}{12} \int_{\mathscr{\mathscr { N }}} \tau_{2}^{3} d \mu_{h}\right. \\
& +\frac{\Lambda}{18} \int_{\mathscr{N}}\left(19 \tau_{2}\left[|\sigma|_{4}^{2}+8 \pi\left(\bar{T}_{r r}\right)_{4}\right]-2\left[|\sigma|_{5}^{2}+8 \pi\left(\bar{T}_{r r}\right)_{5}\right]\right) d \mu_{h} \\
& +\int_{\mathscr{N}}\left(\zeta_{2}+\stackrel{\circ}{R} \tau_{2}\right) d \mu_{h}-2 \Lambda V_{\text {ren }} \\
& =\lim _{r \rightarrow r_{0}} \int_{\mathscr{N}} \zeta d \mu_{\check{g}}+4 \pi \chi(\stackrel{\circ}{\mathscr{N}}) r_{0}+\int_{r=r_{0}}^{\infty} \int_{\mathscr{N}}\left(\frac{1}{2}|\xi|^{2}+S\right) d \mu_{\check{g}} d r .
\end{aligned}
$$


Next we rewrite (5.31) as

$$
\begin{aligned}
16 \pi m_{\mathrm{TB}}= & \int_{\mathscr{\mathscr { N }}}\left(\zeta_{2}+\stackrel{\circ}{R} \tau_{2}\right) d \mu_{h}-\int_{\mathscr{\mathscr { N }}} \frac{\stackrel{\circ}{R}}{2} \tau_{2} d \mu_{h}^{\circ} \\
& +\frac{\Lambda}{3} \int_{\mathscr{\mathscr { N }}}\left(3 \tau_{2}\left[|\sigma|_{4}^{2}+8 \pi\left(\bar{T}_{r r}\right)_{4}\right]-\left[|\sigma|_{5}^{2}+8 \pi\left(\bar{T}_{r r}\right)_{5}\right]\right) d \mu_{h}
\end{aligned}
$$

and find, by (5.42) and (5.43), using $\int_{\mathscr{N}} \stackrel{\circ}{R} d \mu_{h}^{\circ}=4 \pi \chi(\stackrel{\circ}{\mathscr{N}})$,

$$
\begin{aligned}
& 16 \pi m_{\mathrm{TB}}=\lim _{r \rightarrow r_{0}} \int_{\mathscr{N}} \zeta d \mu_{\check{g}}+4 \pi \chi\left(\stackrel{\mathscr{N}}{)} r_{0}+\int_{r=r_{0}}^{\infty} \int_{\mathscr{N}}\left(\frac{1}{2}|\xi|^{2}+S\right) d \mu_{\check{g}} d r\right. \\
& -\int_{\mathscr{N}} \frac{\stackrel{\circ}{R}}{2} \tau_{2} d \mu_{h}+2 \Lambda V_{\text {ren }}+\frac{\Lambda}{12} \int_{\mathscr{N}} \tau_{2}^{3} d \mu_{h}^{\circ} \\
& -\frac{\Lambda}{18} \int_{\mathscr{N}}\left(\tau_{2}\left[|\sigma|_{4}^{2}+8 \pi\left(\bar{T}_{r r}\right)_{4}\right]+4\left[|\sigma|_{5}^{2}+8 \pi\left(\bar{T}_{r r}\right)_{5}\right]\right) d \mu_{h} \text {. }
\end{aligned}
$$

We continue with a generalisation of the arguments leading to equation (43) in [17]. Indeed, we allow the case $r_{0} \neq 0$. Next, for further reference, we allow an asymptotic behaviour for small $r$ for light-cones emanating from a submanifold of general space co-dimension $d$, and not only a light-cone. Finally, for future reference the following calculations, up to the resulting expansion of $\tau$, (5.53), are performed for arbitrary space-time dimensions $n+1 \geq 3$.

Keeping in mind the expansion (2.10) for large $r$, we note that

$$
\tau= \begin{cases}\frac{n-1}{r}+\frac{\tau_{2}}{r^{2}}+O\left(r^{-3}\right), & \text { for large } r \\ \frac{d-1}{r}+O(1), & \text { for small } r .\end{cases}
$$

Here the behaviour for small $r$ is the one which occurs when the set $\{r=0\}$ has space co-dimension $d$ (e.g., $d=n$ for a light-cone emanating from a point). If $r_{0}>0$ we assume that $\tau$ is smooth up-to-boundary when the boundary $r=r_{0}$ is approached.

Next, let

$$
\tau_{1}:=\frac{n-1}{r} .
$$

This is the value of $\tau$ for a light-cone in Minkowski space-time, and it follows from (2.10) that this is the value approached asymptotically along null hypersurfaces meeting $\mathscr{I}$ smoothly and transversally. Let

$$
\delta \tau:=\tau-\tau_{1}
$$

denote the deviation of $\tau$ from its asymptotic value for large $r$, then

$$
\delta \tau= \begin{cases}\frac{\tau_{2}}{r^{2}}+O\left(r^{-3}\right), & \text { for large } r \\ \frac{d-n}{r}+O(1), & \text { for small } r .\end{cases}
$$

(Note that $\delta \tau$ is diverging at the same rate as $\tau$ for small $r$ when $d \neq n$.) From the Raychaudhuri equation (3.11) with $\kappa=0$ one finds that $\delta \tau$ satisfies the equation

$$
\frac{d \delta \tau}{d r}+\left(\frac{\delta \tau}{n-1}+\frac{2}{r}\right) \delta \tau=-|\sigma|^{2}-8 \pi \bar{T}_{r r}
$$


Define

$$
\Psi \equiv r^{-2} \Phi:=r^{-2} \exp \left(\int_{r_{*}}^{r}\left(\frac{\delta \tau}{n-1}+\frac{2}{\tilde{r}}\right) d \tilde{r}\right),
$$

for some $r_{*}$ (possibly depending upon $x^{A}$ ) which will be irrelevant for our final formula (5.53) below except for the requirement that the integral converges. Thus

$$
\frac{1}{\Phi} \frac{d \Phi}{d r}=\frac{\delta \tau}{n-1}+\frac{2}{r}
$$

so that (5.48) is equivalent to

$$
\frac{1}{\Phi} \frac{d(\Phi \delta \tau)}{d r}=-|\sigma|^{2}-8 \pi \bar{T}_{r r}
$$

Using (5.47), we are led to the following three equivalent expressions for the function $\Psi$ :

$$
\Psi\left(r, x^{A}\right)=\left\{\begin{array}{l}
\exp \left(-\int_{r}^{\infty} \frac{\delta \tau}{n-1}\left(s, x^{A}\right) d s+C_{1}\left(x^{A}\right)\right) \\
\exp \left(\int_{r_{0}}^{r} \frac{\delta \tau}{n-1}\left(s, x^{A}\right) d s+C_{2}\left(x^{A}\right)\right) \\
r^{\frac{d-n}{n-1}} \exp \left(\frac{1}{n-1} \int_{0}^{r}\left[\delta \tau\left(s, x^{A}\right)-\frac{d-n}{s}\right] d s+C_{3}\left(x^{A}\right)\right),
\end{array}\right.
$$

for some functions $C_{i}\left(x^{A}\right)$, depending upon the choice of $r_{*}$. In $(5.51 \mathrm{~b})$ we have assumed that $r_{0}>0$, while (5.51c) holds when $\delta \tau\left(r, x^{A}\right) \sim(d-n) r^{-1}$ for small $r$, compare (5.47).

We emphasise that both $\Phi$ and $\Psi$ are auxiliary functions which are only needed to derive (5.53) below, and there is some freedom in their definition. In particular either of the functions $C_{i}\left(x^{A}\right), i=1,2,3$, can be chosen to be zero if convenient for a specific problem at hand, and we note that the $C_{i}\left(x^{A}\right)$ 's cancel out in the final expression for $\tau$ in any case.

We further stress that in the special case of a light-cone we have $d=n$ and treating the case for small $r$ separately is not necessary. In this case (5.51b) coincides with (5.51c).

Thus, using $\delta \tau=\tau-\tau_{1}$ and (5.46),

$$
\frac{\Psi\left(r_{0}, x^{A}\right)}{\Psi\left(r, x^{A}\right)}=\left\{\begin{array}{l}
\exp \left(-\frac{1}{n-1} \int_{r_{0}}^{r}\left(\tau\left(s, x^{A}\right)-\frac{n-1}{s}\right) d s\right) \\
\left(\frac{r_{0}}{r}\right)^{\frac{d-n}{n-1}} \exp \left(\frac{1}{n-1} \int_{r}^{r_{0}}\left(\delta \tau\left(s, x^{A}\right)-\frac{d-n}{s}\right) d s\right)
\end{array}\right.
$$

with both (5.51a) and (5.51b) leading to (5.52a) as long as the right-hand-side of (5.52a) converges, and with (5.52b) holding with $r_{0}=0$ when $\delta \tau\left(r, x^{A}\right) \sim$ $(d-n) r^{-1}$ for small $r$.

Integrating (5.50) and using (5.52a), without denoting the dependence on coordinates $x^{A}$ explicitly in what follows,

$$
\begin{aligned}
\tau= & \frac{n-1}{r}-r^{-2}\left[\Psi(r)^{-1} \int_{\tilde{r}=r_{0}}^{r}\left(|\sigma(\tilde{r})|^{2}+8 \pi \bar{T}_{r r}(\tilde{r})\right) \Psi(\tilde{r}) \tilde{r}^{2} d \tilde{r}\right. \\
& \left.-\lim _{s \rightarrow r_{0}} \frac{\Psi(s)}{\Psi(r)}\left(\tau(s)-\frac{n-1}{s}\right) s^{2}\right] .
\end{aligned}
$$


We can directly read off the expression for $\tau_{2}$ from this:

$$
\begin{aligned}
\tau_{2}= & -\lim _{r \rightarrow \infty}\left\{\Psi(r)^{-1} \int_{\tilde{r}=r_{0}}^{r}\left(|\sigma(\tilde{r})|^{2}+8 \pi \bar{T}_{r r}(\tilde{r})\right) \Psi(\tilde{r}) \tilde{r}^{2} d \tilde{r}\right\} \\
& -\left[\lim _{r \rightarrow \infty} \Psi(r)^{-1}\right] \times \lim _{r \rightarrow r_{0}}\left[\Psi(r)\left(\frac{n-1}{r}-\tau\right) r^{2}\right] .
\end{aligned}
$$

From now on we return to space-time dimension four:

$$
n+1=4 .
$$

Returning to (5.44), inserting the result for $\tau_{2}$ we just found, and using further

$$
d \mu_{\check{g}}=\mathrm{e}^{-\int_{r}^{\infty} \frac{\tilde{r} \tau-2}{\tilde{r}} d \tilde{r}} r^{2} d \mu_{h}
$$

we obtain our final formula for the characteristic mass $m_{\mathrm{TB}}$ of a null hypersurface $\mathscr{N}=\left[r_{0}, \infty\right) \times \stackrel{\mathscr{N}}{\text { : }}$

$$
\begin{aligned}
& m_{\mathrm{TB}}=\frac{1}{16 \pi} \int_{r=r_{0}}^{\infty} \int_{\mathscr{N}}\left(\frac{1}{2}|\xi|^{2}+S\right. \\
& \left.+\left[\frac{\stackrel{\circ}{2}}{2}+\frac{\Lambda}{18}\left(|\sigma|_{4}^{2}+8 \pi\left(\bar{T}_{r r}\right)_{4}\right)\right]\left(|\sigma|^{2}+8 \pi \bar{T}_{r r}\right) \mathrm{e}^{\int_{r}^{\infty} \frac{\tilde{r} \tau-2}{2 \tilde{r}} d \tilde{r}}\right) d \mu_{\check{g}} d r \\
& +\frac{1}{16 \pi}\left[4 \pi \chi(\stackrel{\mathscr{N}}{)}) r_{0}\right. \\
& \left.+\lim _{r \rightarrow r_{0}}\left(\int_{\mathscr{\mathscr { N }}}\left[\zeta+\left(\frac{\stackrel{\circ}{R}}{2}+\frac{\Lambda}{18}\left(|\sigma|_{4}^{2}+8 \pi\left(\bar{T}_{r r}\right)_{4}\right)\right)\left(\frac{2}{r}-\tau\right) \mathrm{e}^{\int_{r_{0}}^{\infty} \frac{r \tau-2}{2 r} d r}\right] d \mu_{\check{g}}\right)\right] \\
& +\frac{\Lambda}{192 \pi} \int_{\mathscr{N}} \tau_{2}^{3} d \mu_{h}+\frac{\Lambda V_{\text {ren }}}{8 \pi}-\frac{\Lambda}{72 \pi} \int_{\mathscr{N}}\left(|\sigma|_{5}^{2}+8 \pi\left(\bar{T}_{r r}\right)_{5}\right) d \mu_{h} .
\end{aligned}
$$

To obtain this equation, it is irrelevant which form of $\Psi$ in (5.51) we take, provided that the same formula is consistently used throughout. For example, if $\Psi(r)$ is given by (5.51a) with $C_{1}\left(x^{A}\right)=0$, then $\lim _{r \rightarrow \infty} \Psi(r)$ equals one, independently of whether $r_{0}=0$ (so that the null hypersurface is singular at $r_{0}$ ) or $r_{0} \neq 0$ (in which case the set $\left\{r=r_{0}\right\}$ has space co-dimension one).

In the special case of a light-cone, where $d=n=3, \stackrel{\circ}{R}=2$ and $r_{0}=0,{ }^{4}$ (5.56) simplifies to

$$
\begin{aligned}
m_{\mathrm{TB}}= & \frac{1}{16 \pi} \int_{0}^{\infty} \int_{\mathscr{N}}\left(\frac{1}{2}|\xi|^{2}+S\right. \\
& \left.+\left[1+\frac{\Lambda}{18}\left(|\sigma|_{4}^{2}+8 \pi\left(\bar{T}_{r r}\right)_{4}\right)\right]\left(|\sigma|^{2}+8 \pi \bar{T}_{r r}\right) \mathrm{e}^{\int_{r}^{\infty} \frac{\tilde{r} \tau-2}{2 \tilde{r}} d \tilde{r}}\right) d \mu_{\check{g}} d r \\
& +\frac{\Lambda}{192 \pi} \int_{\mathscr{N}} \tau_{2}^{3} d \mu_{h}+\frac{\Lambda V_{\mathrm{ren}}}{8 \pi}-\frac{\Lambda}{72 \pi} \int_{\mathscr{N}}\left(|\sigma|_{5}^{2}+8 \pi\left(\bar{T}_{r r}\right)_{5}\right) d \mu_{\grave{h}} \cdot(5.57)
\end{aligned}
$$

\footnotetext{
${ }^{4}$ Recall that $\stackrel{\circ}{R}=2$ when $\stackrel{\circ}{\mathscr{N}}$ is a two-sphere, $\stackrel{\circ}{R}=0$ for a torus, and $\stackrel{\circ}{R}<0$ for higher genus topologies of $\mathscr{I} \approx \mathbb{R} \times \dot{\mathscr{N}}$. In the case of a smooth-light cone the cross-sections are spherical for small $r$, and therefore everywhere, so $\stackrel{\circ}{R}=2$.
} 
Assuming further a conformally smooth compactification and vacuum we have $|\sigma|_{5}^{2}=0$, and after some rearrangements we obtain the striking identity:

$$
\begin{aligned}
m_{\mathrm{TB}}= & \frac{1}{16 \pi} \int_{0}^{\infty} \int_{\mathscr{N}}\left(\frac{1}{2}|\xi|^{2}+|\sigma|^{2} \mathrm{e}^{\int_{r}^{\infty} \frac{\tilde{r} \tau-2}{2 \tilde{r}} d \tilde{r}}\right) d \mu_{\check{g}} d r \\
& +\frac{\Lambda}{8 \pi}\left(V_{\text {ren }}+\frac{1}{12} \int_{\mathscr{\digamma}} \tau_{2}\left(\frac{\tau_{2}^{2}}{2}-\frac{|\sigma|_{4}^{2}}{3}\right) d \mu_{h}^{\circ}\right),
\end{aligned}
$$

with $\tau_{2} \leq 0$ given by

$$
\tau_{2}=-\int_{0}^{\infty}|\sigma|^{2} \mathrm{e}^{-\int_{r}^{\infty} \frac{\tilde{r} \tau-2}{2 \tilde{r}} d \tilde{r}} r^{2} d r .
$$

(Recall that $\tau_{2}=0$ if and only if the metric to the future of $\mathscr{N}$ is, at least locally, the de Sitter or anti-de Sitter metric [6].)

\section{Coordinate mass}

In this section we assume that $\Lambda<0$ and we allow arbitrary space-time dimension $n+1 \geq 4$.

There exist several well-defined notions of mass for asymptotically hyperbolic initial data sets (cf., e.g., $[1,11,16,19,36]$ ), which typically coincide whenever simultaneously defined, some of them defined so forth only in dimension $3+1$. Our aim, in this and in the next section, is to show that the characteristic mass coincides with those alternative definitions in some cases of interest. To set the stage, in this section we introduce the notion of "coordinate mass" for two classes of metrics. (Compare [19, Section V] for a similar treatment in dimension $3+1$.)

\subsection{Birmingham metrics}

Consider an $(n+1)$-dimensional metric, $n \geq 3$, of the form

$$
g=-f(r) d t^{2}+\frac{d r^{2}}{f(r)}+r^{2} \underbrace{\stackrel{\circ}{h}_{A B}\left(x^{C}\right) d x^{A} d x^{B}}_{=: h},
$$

where $\stackrel{\circ}{h}$ is a Riemannian Einstein metric on the compact manifold which, to avoid a proliferation of notation, we will denote as $\mathscr{N}$; we denote by $x^{A}$ the

local coordinates on $\mathscr{\mathscr { N }}$. As discussed in [4], for any $m \in \mathbb{R}$ and $\ell>0$ the function

$$
f=\frac{\stackrel{\circ}{R}}{(n-1)(n-2)}-\frac{2 m}{r^{n-2}}-\varepsilon \frac{r^{2}}{\ell^{2}}, \quad \varepsilon \in\{0, \pm 1\}
$$

where $\stackrel{\circ}{R}$ is the (constant) scalar curvature of $\stackrel{\circ}{h}$, leads to a vacuum metric,

$$
R_{\mu \nu}=\varepsilon \frac{n}{\ell^{2}} g_{\mu \nu}
$$


where the positive constant $\ell$ is related to the cosmological constant as

$$
\frac{1}{\ell^{2}}=\varepsilon \frac{2 \Lambda}{n(n-1)} \text {. }
$$

Clearly, $n$ is not allowed to equal two in (6.2), and we therefore exclude this dimension in what follows.

The multiplicative factor two in front of $m$ is convenient in dimension three when $\stackrel{\circ}{h}$ is a unit round metric on $S^{2}$, and we will keep this form regardless of topology and dimension of $\stackrel{\mathscr{N}}{\text {. }}$

There is a rescaling of the coordinate $r=b \bar{r}$, with $b \in \mathbb{R}^{*}$, which leaves (6.1)-(6.2) unchanged if moreover

$$
\overline{\grave{h}}=b^{2} \stackrel{\circ}{h}, \quad \bar{m}=b^{-n} m, \quad \bar{t}=b t .
$$

We can use this to achieve

$$
\beta:=\frac{\stackrel{\circ}{R}}{(n-1)(n-2)} \in\{0, \pm 1\},
$$

which will be assumed from now on. The set $\{r=0\}$ corresponds to a singularity when $m \neq 0$. Except in the case $m=0$ and $\beta=-1$, by an appropriate choice of the sign of $b$ we can always achieve $r>0$ in the regions of interest. This will also be assumed from now on.

We define

the coordinate mass of the metric (6.1) with $f$ given by (6.2) to be $m$.

Similarly, we define

the coordinate mass of any metric which asymptotes to (6.1)-(6.2) to be $m$.

Here, "asymptotes to" can e.g. be understood as

$$
\begin{aligned}
g= & -\left(f_{m}(R)+o\left(R^{2-n}\right)\right) d T^{2}+\frac{d R^{2}}{\left(f_{m}(R)+o\left(R^{2-n}\right)\right)} \\
& +R^{2}\left(\stackrel{\circ}{h}_{A B}\left(x^{C}\right)+o(1)\right) d x^{A} d x^{B},
\end{aligned}
$$

for large $R$, at fixed $T$, with $f_{m}=f$ given by (6.2).

\subsection{Horowitz-Myers-type metrics}

\subsubsection{The metric}

Consider an $(n+1)$-dimensional metric, $n \geq 3$, of the form

$$
g=f(r) d \psi^{2}+\frac{d r^{2}}{f(r)}+r^{2} \underbrace{}_{=: \stackrel{\circ}{h_{A B}}\left(x^{C}\right) d x^{A} d x^{B}}
$$


where now $\stackrel{\circ}{h}$ is a Riemannian or pseudo-Riemannian Einstein metric on an (n-1)-dimensional manifold $\stackrel{\circ}{N}$ with constant scalar curvature $\stackrel{\circ}{R}$ and, similarly to the last section, the $x^{A}$ 's are local coordinates on $N^{\circ} .^{5}$ This metric can be formally obtained from (6.1) by changing $t$ to $i \psi$. It therefore follows from the discussion of Section 6.1 that for $m \in \mathbb{R}$ and $\ell \in \mathbb{R}^{*}$ the function

$$
f=\beta-\frac{2 m}{r^{n-2}}-\varepsilon \frac{r^{2}}{\ell^{2}}, \quad \varepsilon \in\{0, \pm 1\}, \quad \beta=\frac{\stackrel{\circ}{R}}{(n-1)(n-2)},
$$

leads to a metric satisfying (6.3). Rescaling the coordinate $r$ and the metric $\stackrel{\circ}{h}$ by a suitable constant if necessary we can without loss of generality assume that

$$
\beta \in\{0, \pm 1\} .
$$

Suppose that $f$ has zeros, and let us denote by $r_{0}$ the largest zero of $f$. We assume that $r_{0}$ is of first order, and we restrict attention to $r \geq r_{0}$. Imposing a suitable $\psi_{0}$-periodicity condition on $\psi \in\left[0, \psi_{0}\right]$, the usual arguments imply that the set $\left\{r=r_{0}\right\}$ is a rotation axis in a plane on which $\sqrt{r-r_{0}}$ and $\psi$ are coordinates of polar type: Indeed, if we set

$$
\rho=F(r), \text { with } F=\int_{r_{0}}^{r} \frac{1}{\sqrt{f(r)}} d r=\frac{\sqrt{r-r_{0}}}{2 \sqrt{f^{\prime}\left(r_{0}\right)}}\left(1+O\left(r-r_{0}\right)\right),
$$

we find

$$
\frac{d r^{2}}{f}+f d \psi^{2}=d \rho^{2}+f\left(F^{-1}(\rho)\right) d \psi^{2}=d \rho^{2}+\left(2 f^{\prime}\left(r_{0}\right)\right)^{2}\left(1+O\left(\rho^{2}\right)\right) \rho^{2} d \psi^{2},
$$

which defines a smooth metric near $\rho=0$ if and only if

$$
\psi=\lambda \ell \alpha,
$$

where $\alpha$ is a new $2 \pi$-periodic coordinate, and

$$
\lambda=\frac{1}{2 \ell f^{\prime}\left(r_{0}\right)} .
$$

In the case where

$$
\varepsilon=-1,
$$

one obtains Einstein metrics with a negative cosmological constant.

Whatever $\varepsilon$, a conformal completion at spacelike infinity can be obtained by introducing a new coordinate $x=\ell / r$, bringing $g$ to the form

$$
\begin{aligned}
g & =f\left(\ell x^{-1}\right) \ell^{2} \lambda^{2} d \alpha^{2}+\frac{\ell^{2} d x^{2}}{x^{4} f\left(\ell x^{-1}\right)}+\ell^{2} x^{-2} \stackrel{\circ}{h} \\
& =x^{-2} \ell^{2}\left(-\left(\varepsilon-\beta x^{2}+O\left(x^{n}\right)\right) \lambda^{2} d \alpha^{2}-\left(\varepsilon+\beta x^{2}+O\left(x^{n}\right)\right) d x^{2}+\stackrel{\circ}{h}\right) .
\end{aligned}
$$

We see explicitly that the conformal class of metrics induced by $x^{2} g$ on the boundary at infinity,

$$
\mathscr{I}=\{x=0\} \approx S^{1} \times \stackrel{\circ}{N},
$$

is Lorentzian if $\stackrel{\circ}{h}$ is Lorentzian and if $\varepsilon=-1$.

\footnotetext{
${ }^{5}$ To avoid a proliferation of notation we use the symbol $h$ both for the metric on $\mathscr{N}$ appearing in (6.1) and for the metric on the manifold $\stackrel{\circ}{N}$ relevant for (6.8). Typically $(\stackrel{\circ}{\mathscr{N}}, \stackrel{\circ}{h})$ is a compact Riemannian manifold, while $(\stackrel{\circ}{N}, \stackrel{\circ}{h})$ in $(6.8)$ will be Lorentzian with $\stackrel{\circ}{N}$ noncompact.
} 


\subsection{2 $\beta=0, n=3$}

In [27] Horowitz and Myers consider the case $n+1=4, \varepsilon=-1,{ }^{6}$ and choose $\stackrel{\circ}{h}=-\ell^{-2} d t^{2}+d \varphi^{2}$, with $\varphi$ being a $2 \pi$-periodic coordinate on $S^{1}$. Thus

$$
g=-\frac{r^{2}}{\ell^{2}} d t^{2}+f(r) \ell^{2} \lambda^{2} d \alpha^{2}+\frac{d r^{2}}{f(r)}+r^{2} d \varphi^{2} .
$$

Equation (6.12) shows that timelike infinity $\mathscr{I} \approx \mathbb{R} \times S^{1} \times S^{1}$ is conformally flat:

$$
x^{2} g \rightarrow_{r \rightarrow \infty}-d t^{2}+\ell^{2}\left(\lambda^{2} d \alpha^{2}+d x^{2}+d \varphi^{2}\right) .
$$

Some comments about factors of $\ell$ are in order: if we think of $r$ as having dimension of length, then $\ell, t$ and $\psi$ also have dimension of length, $m$ has dimension length ${ }^{n-1}$, while $f, x$, and the $x^{A}$ 's (and thus $\varphi$ ) are dimensionless.

A uniqueness theorem for the metrics (6.13) has been established in [37].

\subsection{3 $\beta= \pm 1, n=3$}

We consider the metric (6.8) with $^{6} \varepsilon=-1$ and $\stackrel{\circ}{h}$ of the form

$$
\stackrel{\circ}{h}= \begin{cases}d \theta^{2}+\sin ^{2}(\theta) d \varphi^{2}, & \beta=1 \\ d \theta^{2}+\sinh ^{2}(\theta) d \varphi^{2}, & \beta=-1 .\end{cases}
$$

In regions where $f$ is positive, one obtains a Lorentzian metric after a "double Wick rotation"

$$
\theta=i \ell^{-1} t, \varphi=i \phi
$$

resulting in

$$
g=-\frac{r^{2}}{\ell^{2}} d t^{2}+\frac{d r^{2}}{f(r)}+f(r) \ell^{2} \lambda^{2} d \alpha^{2}+r^{2} \begin{cases}\sinh ^{2}\left(\ell^{-1} t\right) d \phi^{2}, & \beta=1 \\ \sin ^{2}\left(\ell^{-1} t\right) d \phi^{2}, & \beta=-1 .\end{cases}
$$

Taking $\alpha$ and $\phi$ periodic one obtains again a conformal infinity diffeomorphic to $\mathbb{R} \times \mathbb{T}^{2}$. Note that the conformal metric at the conformal boundary is not conformally stationary anymore, as opposed to the Horowitz-Myers metrics (6.14). We have not attempted to study the nature of the singularities of $g$ at $\sinh \left(\ell^{-1} t\right)=0$ or at $\sin \left(\ell^{-1} t\right)=0$.

\subsubsection{Negative coordinate mass}

For completeness, we show that the metric (6.8) has the striking property that its total coordinate mass is negative when $m$ is positive; the latter is needed for regularity of the metric. This has already been observed in [27] in spacedimension three with a toroidal Scri. Here we check that this remains correct in higher dimensions, for a large class of topologies of Scri.

Before continuing, we note that Lorentzian Horowitz-Myers-type metrics with a smooth conformal compactification at infinity exist only with negative

\footnotetext{
${ }^{6}$ The case $\beta=0$ and $\varepsilon=1$ leads to a signature $(+---)$ for large $r$; our signature $(-+++)$ is recovered by multiplying the metric by minus one, but then one is back in the case $\varepsilon=-1$ after renaming $m$ to $-m$.
} 
$\Lambda$ : Indeed, to obtain the right signature for large $r$ when $\epsilon>0$ one needs to multiply the metric by minus one. But then the resulting metric has negative Ricci scalar, and hence solves Einstein equations with a negative cosmological constant.

Somewhat more generally, consider those metrics of the form (6.8) for which

$$
\stackrel{\circ}{N}=\mathbb{R}_{t} \times \check{N},
$$

where $(\check{N}, \check{h})$ is a compact Riemannian manifold, and where

$$
\stackrel{\circ}{h}=-\ell^{-2} d t^{2}+\check{h},
$$

so that

$$
g=f(r) d \psi^{2}+\frac{d r^{2}}{f(r)}+r^{2}\left(-\ell^{-2} d t^{2}+\check{h}\right) .
$$

The question arises, how to define the mass of such a metric.

To avoid ambiguities, let us write $f_{m}$ for the function $f$ of (6.9).

To assign a coordinate mass to a metric (6.18), we need to check whether metrics satisfying (6.8)-(6.9) and (6.17) can be written in the form (6.7) by setting $r=r(\rho)$ :

$$
\begin{aligned}
g= & f_{m}(r) \ell^{2} \lambda^{2} d \alpha^{2}+\frac{d r^{2}}{f_{m}(r)}+\frac{r^{2}}{\ell^{2}}\left(-d t^{2}+\ell^{2} \check{h}\right) \\
= & -\frac{r^{2}}{\ell^{2}} d t^{2}+\left(\frac{d r}{d \rho}\right)^{2} \frac{d \rho^{2}}{f_{m}(r)} \\
& +r^{2}\left(\left(1+O\left(\beta r^{-2}\right)+O\left(m r^{-n}\right)\right) \lambda^{2} d \alpha^{2}+\check{h}\right),
\end{aligned}
$$

where the error terms have to be understood for large $r$. We will have

$$
g \approx-f_{M}(\rho) d t^{2}+\frac{d \rho^{2}}{f_{M}(\rho)}+\rho^{2}\left(\lambda^{2} d \alpha^{2}+\check{h}\right),
$$

for some parameter $M$ possibly different from $m$, provided that

$$
r^{2}=\ell^{2} f_{M}(\rho)\left(1+o\left(\rho^{-n}\right)\right), \quad\left(\frac{d \rho}{d r}\right)^{2} f_{m}(r)=f_{M}(\rho)\left(1+o\left(\rho^{-n}\right)\right),
$$

The first equation determines $r$ as a function of $\rho$ up to correction terms $o\left(\rho^{-n}\right)$. Inserting the result into the second equation determines $M$, provided that the asymptotic expansion of the left-hand side is compatible with that of the righthand side. However, it is straightforward to check that these equations are compatible if and only if

$$
\beta=0 .
$$

We conclude that for metrics satisfying (6.8)-(6.9) and (6.17)

the coordinate mass is only defined if $\beta=0$.

Assuming (6.21), after asymptotically solving the first equation in (6.20) and inserting the result into the second one, we find that

$$
\rho=r+\frac{\ell^{2} M}{r^{n-1}}+O\left(r^{-(2 n-1)}\right),
$$


and that the coordinate mass equals

$$
M=-\frac{m}{n-1} .
$$

In particular $M$ is negative for positive $m$.

\section{Examples}

Throughout this section we allow arbitrary space-time dimension $n+1 \geq 4$. We show that the numerical value of the Trautman-Bondi mass, as generalised to higher dimensions below, and which coincides with the characteristic mass defined in Section 5 in dimension $3+1$, is proportional to the "coordinate mass" for the metrics considered in Section 6. This, in itself, is not surprising, since these metrics have only the mass parameter $m$ as free parameter, so whatever we will calculate must be a function of $m$. The main conclusion here appears to be that $m_{\mathrm{TB}}$ is a linear function of $m$, with a strictly positive proportionality factor. A full agreement will be obtained in the analysis of the Hamiltonian mass in Section 8 below, where the proportionality factors will also be matched.

In what follows, we seek to write the metrics under consideration in the form (2.2),

$$
g=g_{u u} d u^{2}-2 e^{2 \omega} d r d u-2 r^{2} U_{A} d x^{A} d u+r^{2} \underbrace{h_{A B} d x^{A} d x^{B}}_{=: h},
$$

where the determinant of $h_{A B}$ is $r$-independent. By analogy with (5.1)-(5.2), in space-time dimension $n+1$ we set

$$
m_{\mathrm{TB}}=\frac{1}{8 \pi} \lim _{r \rightarrow \infty} \int_{\mathscr{N}}\left(g_{u u}\right)_{n-2} d \mu_{h} .
$$

This definition is motivated by the fact that, when solving the characteristic constraint equations on a null hypersurface, the $\left(g_{u u}\right)_{n-2}$-coefficient in the expansion of $g_{u u}$ arises as a global integration function.

\subsection{Birmingham metrics}

Consider, first, the original Birmingham metrics (6.1),

$$
g=-f(r) d t^{2}+\frac{d r^{2}}{f(r)}+r^{2} \stackrel{\circ}{h}
$$

with $f$ given by (6.2). Introducing a new coordinate $u=t-\int_{r *}^{r} f^{-1}(s) d s$, for some conveniently chosen $r_{*}$, brings $g$ to the desired form

$$
\begin{aligned}
g= & -f(r)\left(d u+\frac{d r}{f(r)}\right)^{2}+\frac{d r^{2}}{f(r)}+r^{2} \stackrel{\circ}{R} \\
= & -\left(\frac{\left.\stackrel{\circ}{(n-1)(n-2)}-\frac{2 m}{r^{n-2}}-\varepsilon \frac{r^{2}}{\ell^{2}}\right) d u^{2}-2 d u d r+r^{2} \stackrel{\circ}{h}}{},\right.
\end{aligned}
$$

where $\varepsilon \in\{ \pm 1\}$ is the sign of the cosmological constant $\Lambda=\varepsilon|\Lambda|$, which we allow to be either positive or negative. 


\subsubsection{Mass and volume}

The coordinate $r$ provides obviously a radial Bondi coordinate. Moreover, the equality $\partial_{r}=-\nabla u$ implies that $r$ is also an affine parameter along the radial null outgoing geodesics of $g$. When $m=0$ we have the explicit formulae

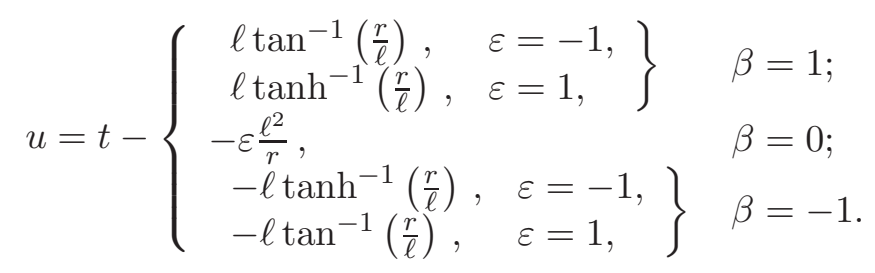

Equation (7.2) leads to a Bondi-Trautman-type mass

$$
m_{\mathrm{TB}}=\frac{m}{4 \pi} \int_{\mathscr{N}} d \mu_{h} \equiv m \frac{\mu_{\grave{h}}(\stackrel{\mathscr{N}}{ })}{4 \pi} .
$$

(Here the normalisation factor $8 \pi$ in $(7.2)$ is clearly convenient only when $\mathscr{N}$ is a unit round two-dimensional sphere, but this issue will be of no concern to us here.) We conclude that the characteristic mass of null hypersurfaces asymptotic to the level sets of $t$ is indeed proportional to the coordinate mass, with a positive proportionality factor. We will see in Section 8.1 that the proportionality factor is the same as the one occurring in the Hamiltonian definition of mass, see (8.34) below.

\subsubsection{The balance equation for Birmingham metrics}

Consider metrics of the form

$$
g=-f(r) d t^{2}+\frac{d r^{2}}{f(r)}+r^{2} \underbrace{\stackrel{\circ}{h}_{A B} d x^{A} d x^{B}}_{=: h} .
$$

Recall (7.4): setting $u=t-\int_{r *}^{r} f^{-1}(s) d s$, for some conveniently chosen $r_{*}$, brings $g$ to a Bondi form provided that $\operatorname{det} \stackrel{\circ}{h}$ is $r$-independent:

$$
g=-f d u^{2}-2 d u d r+r^{2} h .
$$

The inverse metric reads

$$
g^{\sharp} \equiv g^{\mu \nu} \partial_{\mu} \partial_{\nu}=f \partial_{r}^{2}-2 \partial_{u} \partial_{r}+r^{-2} \stackrel{\circ}{h}^{\sharp},
$$

where $h^{\sharp}=\stackrel{\circ}{h}^{A B} \partial_{A} \partial_{B}$ is the metric inverse to $\stackrel{\circ}{\text {. }}$

Similarly to the previous section, the integral curves of the vector field

$$
-\nabla u=\partial_{r}
$$

are affinely parameterized geodesics. Whenever $\operatorname{det} \stackrel{\circ}{h}$ is $r$-independent, the function $r$ is therefore both an area coordinate and an affine parameter along the generators of the null hypersurfaces $\{u=$ const $\}$. 
Recall that $V_{\text {ren }}$ is defined as the limit, as $r$ approaches infinity, of the volume $V(r)$ of the light-cone minus all diverging terms in an asymptotic expansion of $V(r)$ :

$$
\begin{aligned}
V_{\text {ren }}= & \lim _{r \rightarrow \infty}\left\{V(r)+\frac{1}{2}\left[-\frac{2}{3} r^{3} \int_{\mathscr{N}} d \mu_{h}+r^{2} \int_{\mathscr{N}} \tau_{2} d \mu_{h}+r \int_{\mathscr{N}}\left(|\sigma|_{4}^{2}-\frac{1}{2} \tau_{2}^{2}\right) d \mu_{h}\right.\right. \\
& \left.\left.+\frac{1}{3} \log r \int_{\mathscr{N}}\left(|\sigma|_{5}^{2}-2|\sigma|_{4}^{2} \tau_{2}\right) d \mu_{h}^{\circ}\right]\right\}<\infty
\end{aligned}
$$

(for simplicity a metric vacuum to sufficiently high order has been assumed in $(7.8))$.

For the Birmingham metrics (6.13) we have $T_{r r} \equiv 0 \equiv S \equiv \sigma \equiv \xi \equiv \tau_{2}$, $\stackrel{\circ}{R}=2$, and the volume function is straightforward:

$$
\begin{aligned}
V(r) & =\int_{r_{*}}^{r} \int_{\mathscr{N}} \sqrt{\operatorname{det} g_{A B}} d^{2} x d s \\
& =\frac{\mu_{h}(\mathscr{N})}{3}\left(r^{3}-r_{*}^{3}\right) \Longrightarrow V_{\text {ren }}=-\frac{\mu_{h}(\mathscr{N}) r_{*}^{3}}{3} .
\end{aligned}
$$

The mass formula (5.10) reduces to

$$
m_{\mathrm{TB}}=\frac{1}{16 \pi}\left(4 \pi \chi\left(\stackrel{\mathscr{N}}{)} r_{*}+\lim _{r \rightarrow r_{*}} \int_{\mathscr{\mathscr { N }}} \zeta d \mu_{\check{g}}\right)+\frac{\Lambda V_{\mathrm{ren}}}{8 \pi}\right.
$$

Note that this holds for any value of $r_{*}$. A natural choice would be to choose $r_{*}$ to be the location of the outermost past horizon, but we allow $r_{*}$ to be arbitrary.

Specialising to the Birmingham metrics we find, in space-time dimension $n+1=4$,

$$
\begin{aligned}
& 16 \pi m_{\mathrm{TB}}=4 \pi \chi(\stackrel{\circ}{\mathscr{N}}) r_{*}+\lim _{r \rightarrow r_{*}} \mu_{h}(\stackrel{\circ}{\mathscr{N}})\left(-2 r^{2}\left(\frac{\beta}{r}-\frac{2 m}{r^{2}}+\frac{r}{\ell^{2}}\right)-\frac{2 \Lambda r^{3}}{3}\right) \\
& =4 \pi \chi(\stackrel{\circ}{\mathscr{N}}) r_{*}+\mu_{h}\left(\stackrel{\mathscr{N}}{)}\left(-2 r_{*} \beta+4 m\right)\right. \\
& =4 \mu_{h}(\stackrel{\circ}{\mathscr{N}}) m \text {, }
\end{aligned}
$$

where we have used the Gauss-Bonnet theorem to cancel the term containing the Euler characteristic $\chi(\stackrel{\mathscr{N}}{)})$ of $\mathscr{\mathscr { N }}$ with the term involving $\beta$.

\subsection{Horowitz-Myers type metrics}

We pass now to the metrics (6.8) with $\stackrel{\circ}{h}$ given by (6.17) and $\psi$ replaced by $\lambda \ell \alpha$, with $\lambda$ given by (6.11), and where $\alpha$ is $2 \pi$-periodic:

$$
\begin{aligned}
g & =-\frac{r^{2}}{\ell^{2}} d t^{2}+\frac{d r^{2}}{f}+f \ell^{2} \lambda^{2} d \alpha^{2}+r^{2} \check{h} \\
& =-\frac{r^{2}}{\ell^{2}} d t^{2}+\frac{d r^{2}}{f}+\underbrace{r^{\frac{2(n-2)}{n-1}}\left(f \ell^{2}\right)^{\frac{1}{n-1}}}_{=: r_{\text {Bo }}^{2}} \underbrace{\left(\frac{f \ell^{2} \lambda^{2}}{r_{\text {Bo }}^{2}} d \alpha^{2}+\frac{r^{2}}{r_{\text {Bo }}^{2}} \check{h}\right)}_{r \text {-independent determinant }} .
\end{aligned}
$$

We want $f$ to be positive for large $r$, and hence we need to assume that $\Lambda<0$. 


\subsubsection{Bondi coordinates, characteristic mass}

Setting $d u=d t-\ell f^{-1 / 2} r^{-1} d r$ we obtain

$$
\begin{aligned}
g & =-\frac{r^{2}}{\ell^{2}} d u^{2}-\frac{2 r}{f^{1 / 2} \ell} d u d r+r_{\mathrm{Bo}}^{2}\left(\frac{f \ell^{2} \lambda^{2}}{r_{\mathrm{Bo}}^{2}} d \alpha^{2}+\frac{r^{2}}{r_{\mathrm{Bo}}^{2}} \check{h}\right) \\
& =-\frac{r^{2}}{\ell^{2}} d u^{2}-\frac{2 r}{f^{1 / 2} \ell}\left(\frac{d r}{d r_{\mathrm{Bo}}}\right) d u d r_{\mathrm{Bo}}+r_{\mathrm{Bo}}^{2}\left(\frac{f \ell^{2} \lambda^{2}}{r_{\mathrm{Bo}}^{2}} d \alpha^{2}+\frac{r^{2}}{r_{\mathrm{Bo}}^{2}} \check{h}\right) .
\end{aligned}
$$

Note that the we have obtained Bondi coordinates only if the determinant $\breve{h}$ is $t$-independent, as otherwise the replacement of $t$ by its expression in terms of $u$ and $r$ introduces back $r$-dependence in the determinant of $g_{A B}$. Equation (6.16) clearly shows that this requires $\beta=0$ in dimension $n+1=4$. Nevertheless we continue our calculations without assuming the vanishing of $\beta$.

In space-time dimension $n+1=4$ we find

$$
r_{\mathrm{Bo}}=r+\frac{\beta \ell^{2}}{4 r}-\frac{\ell^{2} m}{2 r^{2}}+O\left(r^{-3}\right) \Longleftrightarrow r=r_{\mathrm{Bo}}-\frac{\beta \ell^{2}}{4 r_{\mathrm{Bo}}}+\frac{\ell^{2} m}{2 r_{\mathrm{Bo}}^{2}}+O\left(r_{\mathrm{Bo}}^{-3}\right),
$$

leading to

$$
g_{u u}=-\left(\frac{r_{\mathrm{Bo}}^{2}}{\ell^{2}}-\frac{\beta}{2}+\frac{m}{r_{\mathrm{Bo}}}+\frac{\beta^{2} \ell^{2}}{16 r_{\mathrm{Bo}}^{2}}\right)+O\left(r_{\mathrm{Bo}}^{-3}\right) .
$$

Equation (7.2) gives

$$
m_{\mathrm{TB}}=-\frac{m \mu_{\hat{h}}\left(S^{1} \times \check{N}\right)}{8 \pi},
$$

where $\mu_{\hat{h}}$ is the measure induced on $S^{1} \times \check{N}$ by the metric

$$
\hat{h}:=\lim _{r \rightarrow \infty}\left(\frac{f \ell^{2} \lambda^{2}}{r_{\text {Bo }}^{2}} d \alpha^{2}+\frac{r^{2}}{r_{\text {Bo }}^{2}} \check{h}\right)=\lambda^{2} d \alpha^{2}+\check{h} .
$$

In all dimensions, when $\beta$ vanishes we find

$$
m_{\mathrm{TB}}=-\frac{m \mu_{\hat{h}}\left(S^{1} \times \check{N}\right)}{4(n-1) \pi},
$$

and the above remains true whether or not $\beta$ vanishes in odd space-dimensions $n$. We see that in these case the characteristic mass coincides with the coordinate mass, up to a volume normalisation factor related to the integrals involved. We will see in Section 8.2 that, similarly to the Birmingham metrics, in spacetime dimension four the proportionality coefficient is the same as that for the Hamiltonian mass, see (8.51) below.

In even space-dimensions $n=2 k$, when $\beta$ does not vanish, a calculation shows that the definition (7.2) gives instead the curious formula

$$
m_{\mathrm{TB}}=-\frac{\left(m+c_{k} \beta^{k} \ell^{n-2}\right) \mu_{\hat{h}}\left(S^{1} \times \check{N}\right)}{4(n-1) \pi},
$$

where $c_{k} \in \mathbb{R}^{*}$ is a numerical coefficient depending upon $k$. For example, we have

$$
c_{2}=\frac{1}{6}, c_{3}=-\frac{2}{25}, c_{4}=\frac{33}{686}, c_{5}=-\frac{644}{19683}, c_{6}=\frac{7735}{322102} .
$$




\subsubsection{Renormalized volume}

With the choice

$$
\psi_{0}=2 \pi \ell \lambda,
$$

where $\lambda$ is given by (6.11), the curves obtained by letting $\psi$ vary from zero to $\psi_{0}$ while keeping $t$ fixed and $r=r_{0}$, where $f\left(r_{0}\right)=0$ with $f$ given by (6.9), are closed geodesics for the metric (6.8): This follows from the fact that the manifold $\left\{r=r_{0}\right\}$ is the fixed-point set of the group of isometries generated by the Killing vector field $\partial_{\psi}$, and is therefore totally geodesic. Those geodesics will be referred to as core geodesics, or emission curves.

From the definition of $r_{0}$ we have

$$
\frac{r_{0}^{2}}{\ell^{2}}=\frac{2 m}{r_{0}} \quad \Longleftrightarrow \quad r_{0}=\left(2 m \ell^{2}\right)^{\frac{1}{3}}
$$

It is remarkable that the null surfaces issuing normally from those geodesics are smooth away from the emission curves, and their union covers the whole space-time.

The contravariant metric $g^{\sharp}$ associated to (7.11) equals

$$
g^{\sharp}=f \partial_{r}^{2}-\frac{2 \ell \sqrt{f(r)}}{r} \partial_{u} \partial_{r}+f^{-1} \ell^{-2} \lambda^{-2} \partial_{\alpha}^{2}+r^{-2} \partial_{\varphi}^{2} .
$$

The vector field

$$
-\nabla u=\frac{\ell \sqrt{f(r)}}{r} \partial_{r}
$$

has vanishing Lorentzian length, and a standard argument shows that its integral curves are affinely parameterized geodesics. Hence the parameter $s$ defined as

$$
\frac{d s}{d r}=\frac{r}{\ell \sqrt{f(r)}} \quad \Longleftrightarrow \quad \partial_{s}=\frac{\ell \sqrt{f(r)}}{r} \partial_{r}
$$

is an affine parameter along the generators of the null hypersurfaces $\{u=$ const $\}$. (An explicit expression for $s$ in terms of elliptic integrals in space-time dimension $n+1=4$ can be given, which again does not appear to be very useful.)

We are ready to calculate the renormalized volume $V_{\text {ren }}$. We have

$$
\begin{aligned}
V\left(s^{\prime}\right) & =\int_{s=0}^{s^{\prime}} \int_{\mathscr{N}} \sqrt{\operatorname{det} g_{A B}} d^{2} x d s=\int_{s=0}^{s^{\prime}} \int_{\mathscr{N}} \ell \lambda \sqrt{f} r d \alpha d \varphi \underbrace{\frac{d s}{d r}}_{\frac{r}{\ell \sqrt{f(r)}}} d r \\
& =\mu_{\hat{h}}(\stackrel{\mathscr{N}}{)}) \int_{r(0)}^{r\left(s^{\prime}\right)} r^{2} d r=\frac{1}{3} \mu_{\hat{h}}(\stackrel{\mathscr{N}}{ })\left(r^{3}\left(s^{\prime}\right)-r_{0}^{3}\right) .
\end{aligned}
$$

Here one should keep in mind that $r^{3}\left(s^{\prime}\right)$ needs to be reexpressed in terms of the affine parameter $s^{\prime}$ before removing the singular part of $V\left(s^{\prime}\right)$. For this, integration of (7.22) gives, for large $r$,

$$
s=r-r_{0}+\underbrace{\int_{r_{0}}^{\infty}\left(\frac{r}{\ell \sqrt{f}}-1\right) d r}_{=: s_{*}}-\frac{\ell^{2} m}{2 r^{2}}-\frac{3 \ell^{4} m^{2}}{10 r^{5}}+O\left(r^{-8}\right),
$$


with $0<s_{*}<\infty$ for $m>0$.

It is convenient to introduce a dimensionless variable $x$ through the formula $s=r_{0} x=\left(2 m \ell^{2}\right)^{1 / 3} x$; set $s_{*}=\left(2 m \ell^{2}\right)^{1 / 3} x_{*}$. After inverting (7.24) one obtains $r^{3}-r_{0}^{3}=\frac{1}{2} \ell^{2} m\left(4 x^{3}-12 x^{2}\left(x_{*}-1\right)+12 x\left(x_{*}-1\right)^{2}-4 x_{*}^{3}+12 x_{*}^{2}-12 x_{*}+3\right)$.

Inserting into (7.23) leads to

$$
\begin{aligned}
V_{\text {ren }} & =\frac{1}{6} \ell^{2} m\left(-4 x_{*}^{3}+12 x_{*}^{2}-12 x_{*}+3\right) \mu_{\hat{h}}(\stackrel{\mathscr{N}}{)}) \\
& =\frac{1}{6} \ell^{2} m \underbrace{\left(4\left(1-x_{*}\right)^{3}-1\right)}_{\approx-0.6793} \mu_{\hat{h}}(\stackrel{\mathscr{N}}{)})
\end{aligned}
$$

where we have used

$$
x_{*}=\int_{1}^{\infty}\left(\frac{1}{\sqrt{1-\frac{1}{x^{3}}}}-1\right) d x \approx 0.568815 .
$$

More information on the null geometry of Horowitz-Myers metrics, as well as a term-by-term analysis of the balance equation, can be found in Appendix A.

\section{Hamiltonian mass, $\Lambda<0$}

Until specified otherwise, we allow arbitrary space-time dimension $n+1 \geq 4$.

The calculations of the mass so far might appear to be ad-hoc. In particular one wonders, why the coordinates approach of Section 6.2.4 appears to allow only the $\beta=0$ case for HM-type metrics. As such, a systematic way of obtaining an expression for the energy of a field configuration is to use a Hamiltonian approach. Now, both families of metrics (6.1) and (6.18), with $f$ given by (6.2), are asymptotic, as $r \rightarrow \infty$, to a background metric $b$ obtained by setting $m=0$ in $f$ (with different backgrounds for each family). When $\Lambda<0$ one can therefore use, ${ }^{7}$ in each case, the formalism of [9] (as already done in [19] for $(3+1)$-dimensional asymptotically Kottler metrics), to define the mass of $g$ relative to $b$. Indeed, the Hamiltonian analysis in [9] shows that to every spacelike hypersuface $\mathscr{S}$ and $b$-Killing vector $X$ one can associate a Hamiltonian mass $H(X, \mathscr{S})$ through the formula

$$
H(X, \mathscr{S})=\frac{1}{2} \int_{\partial \mathscr{S}} \mathbb{U}^{\alpha \beta} d S_{\alpha \beta},
$$

where the integral over $\partial \mathscr{S}$ is understood as the limit of integrals over a family of well behaved boundaries of sets which exhaust $\mathscr{S}$. Here $d S_{\alpha \beta}$ is defined as $\left.\left.\frac{\partial}{\partial x^{\alpha}}\right\lrcorner \frac{\partial}{\partial x^{\beta}}\right\lrcorner d x^{0} \wedge \cdots \wedge d x^{n}$, with $\lrcorner$ denoting contraction, and $\mathbb{U}^{\alpha \beta}$ is given by

$$
\begin{aligned}
\mathbb{U}^{\nu \lambda} & =\mathbb{U}_{\beta}^{\nu \lambda} X^{\beta}+\frac{1}{8 \pi} \Delta^{\alpha[\nu} X_{; \alpha}^{\lambda]}, \\
\mathbb{U}_{\beta}^{\nu \lambda} & =\frac{2\left|\operatorname{det} b_{\mu \nu}\right|}{16 \pi \sqrt{\left|\operatorname{det} g_{\rho \sigma}\right|}} g_{\beta \gamma}\left(e^{2} g^{\gamma[\nu} g^{\lambda] \kappa}\right)_{; \kappa},
\end{aligned}
$$

\footnotetext{
${ }^{7}$ When $\Lambda>0$ a Hamiltonian definition of mass requires somewhat different considerations, see $[12]$.
} 
where a semicolon denotes covariant differentiation with respect to the background metric $b$, while

$$
\begin{gathered}
e:=\frac{\sqrt{\left|\operatorname{det} g_{\rho \sigma}\right|}}{\sqrt{\left|\operatorname{det} b_{\mu \nu}\right|}}, \\
\Delta^{\alpha \nu}:=\frac{\sqrt{\left|\operatorname{det} g_{\rho \sigma}\right|}}{\sqrt{\left|\operatorname{det} b_{\alpha \beta}\right|}} g^{\alpha \nu}-b^{\alpha \nu .} .
\end{gathered}
$$

\subsection{Asymptotically Birmingham metrics}

We wish, first, to calculate $(8.1)$ for $(n+1)$ dimensional metrics with Birmingham asymptotics, with a negative cosmological constant $\Lambda$ (equivalently, in (6.2) we take $\varepsilon=-1$ ), with the Killing vector $\partial_{0}$ and with $\Sigma=\{t=$ const $\}$. For this, it is useful to introduce the following $b$-orthonormal frame:

$$
e_{\hat{0}}=\frac{1}{\sqrt{\beta+\frac{r^{2}}{\ell^{2}}}} \partial_{0}, \quad e_{\hat{1}}=\sqrt{\beta+\frac{r^{2}}{\ell^{2}}} \partial_{r}, \quad e_{\hat{A}}=\frac{1}{r} \iota_{\hat{A}},
$$

where $\iota_{\hat{A}}$ is an orthonormal (ON) frame for the metric $\stackrel{\circ}{h}$. To avoid ambiguities: the contravariant form of the background metric is, by definition

$$
b^{\mu \nu} \partial_{\mu} \otimes \partial_{\nu}:=-e_{\hat{0}} \otimes e_{\hat{0}}+e_{\hat{1}} \otimes e_{\hat{1}}+\sum_{A=2}^{n} e_{\hat{A}} \otimes e_{\hat{A}} .
$$

Here and in what follows in the current section we use

$$
A \in\{2,3, \ldots, n\},
$$

and we shall use hatted indices to denote the components of a tensor field in the frame $e_{\hat{\mu}}$ defined in (8.6). The connection coefficients, defined as $\nabla_{e_{\hat{\mu}}} e_{\hat{\nu}}=$ $\omega^{\hat{\rho}} \hat{\nu} \hat{\mu} e_{\hat{\rho}}$ with $\nabla$ associated with $b$, read

$$
\begin{gathered}
\omega_{\hat{0} \hat{1} \hat{O}}=-\frac{r}{\ell^{2} \sqrt{\beta+\frac{r^{2}}{\ell^{2}}}}=-\frac{1}{\sqrt{\beta \frac{\ell^{4}}{r^{2}}+\ell^{2}}}=-\frac{1}{\ell}+O\left(r^{-2}\right), \\
\omega_{\hat{1} \hat{A} \hat{B}}=-\frac{\sqrt{\beta+\frac{r^{2}}{\ell^{2}}}}{r} b_{\hat{A} \hat{B}}=-\sqrt{\frac{\beta}{r^{2}}+\frac{1}{\ell^{2}}} b_{\hat{A} \hat{B}}=\left(-\frac{1}{\ell}+O\left(r^{-2}\right)\right) b_{\hat{A} \hat{B}} .
\end{gathered}
$$

The remaining possibly non-vanishing connection coefficients, not obtained from the above by permutations of indices, are the $\omega_{\hat{A} \hat{B} \hat{C}}$ 's, with $\hat{A} \neq \hat{B}$. For example, in space-time dimension $3+1$, if we use a coordinate system $\theta, \varphi$ on

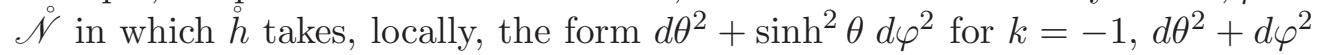
for $k=0$, and $d \theta^{2}+\sin ^{2} \theta d \varphi^{2}$ for $k=1$, we find

$$
\omega_{\hat{2} \hat{3} \hat{3}}= \begin{cases}-\frac{\operatorname{coth} \theta}{r}, & k=-1 \\ 0, & k=0 \\ -\frac{\cot \theta}{r}, & k=1 .\end{cases}
$$

However, the exact form above, and the one of $\omega_{\hat{A} \hat{B} \hat{C}}$ in general, is not needed for what follows. 
We further have

$$
\begin{gathered}
X^{\hat{0}}=\sqrt{\beta+\frac{r^{2}}{\ell^{2}}}=\frac{r}{\ell}+O\left(r^{-1}\right), \\
e_{\hat{1}}\left(X^{\hat{0}}\right)=\left(X^{\hat{0}}\right)_{; \hat{1}}=-X_{\hat{0} ; \hat{1}}=X_{\hat{1} ; \hat{0}}=\frac{r}{\ell^{2}},
\end{gathered}
$$

with the third equality in (8.11) following from the Killing equations $X_{\mu ; \nu}+$ $X_{\nu ; \mu}=0$; all the remaining $X^{\hat{\mu}}$ 's and $X_{\hat{\mu} ; \hat{\nu}}$ 's are zero.

Let the tensor field $e^{\mu \nu}$ be defined by the formula

$$
e^{\mu \nu}:=g^{\mu \nu}-b^{\mu \nu}
$$

As already mentioned, we use hatted indices to denote the components of a tensor field in the frame $e_{\hat{\mu}}$, e.g. $e^{\hat{\mu} \hat{\nu}}$ denotes the coefficients of $e^{\mu \nu}$ with respect to that frame:

$$
e^{\mu \nu} \partial_{\mu} \otimes \partial_{\nu}=e^{\hat{\mu} \hat{\nu}} e_{\hat{\mu}} \otimes e_{\hat{\nu}}
$$

Let the $\theta^{\hat{A}}$ 's form a coframe dual to the $e_{\hat{A}}$ 's. Then

$$
\theta^{\hat{0}} \wedge \ldots \wedge \theta^{\hat{n}}=\sqrt{\left|\operatorname{det} b_{\alpha \beta}\right|} d x^{0} \wedge \ldots \wedge d x^{n},
$$

and so on the level sets of $t$ intersected with those of $r$ we have

$$
\begin{aligned}
\left.\mathbb{U}^{\alpha \beta} d S_{\alpha \beta}\right|_{r=R} & \left.\left.=\mathbb{U}^{\hat{\alpha} \hat{\beta}} e_{\hat{\alpha}}\right\rfloor e_{\hat{\beta}}\right\rfloor\left.\left(d x^{0} \wedge \ldots \wedge d x^{n}\right)\right|_{r=R} \\
& \left.\left.=\frac{\mathbb{U}^{\hat{\alpha} \hat{\beta}}}{\sqrt{\left|\operatorname{det} b_{\alpha \beta}\right|}} e_{\hat{\alpha}}\right\rfloor e_{\hat{\beta}}\right\rfloor\left.\left(\theta^{\hat{0}} \wedge \ldots \wedge \theta^{\hat{n}}\right)\right|_{r=R} \\
& =\left.\frac{2 \mathbb{U}^{\hat{1} \hat{0}}}{\sqrt{\left|\operatorname{det} b_{\alpha \beta}\right|}} \theta^{\hat{2}} \wedge \ldots \wedge \theta^{\hat{n}}\right|_{r=R} .
\end{aligned}
$$

From (8.1) we thus find

$$
H(X, \mathscr{S})=\lim _{R \rightarrow \infty} \int_{\mathscr{S} \cap\{r=R\}} \frac{\mathbb{U}^{\hat{1} \hat{0}}}{\sqrt{\left|\operatorname{det} b_{\alpha \beta}\right|}} \theta^{\hat{2}} \wedge \ldots \wedge \theta^{\hat{n}} .
$$

We wish to analyze when the above limit exists. Since every $\theta^{\hat{A}}$ comes with a multiplicative factor of $r$ in local coordinates on the level sets of $R$ within $\mathscr{S}$, again in local coordinates the integrand in (8.13) behaves as $r^{n-1} \mathbb{U}^{\hat{1} \hat{0}}$. Now,

$$
r^{n-1} \mathbb{U}^{\hat{1} \hat{0}} X^{\beta}=r^{n-1} \mathbb{U}^{\hat{1} \hat{0}}{ }_{0} X^{\hat{0}} \approx \frac{r^{n}}{\ell} \mathbb{U}^{\hat{1} \hat{0}} \hat{0},
$$

hence in the calculations we only need to keep track of those terms in $\mathbb{U}^{\hat{1} \hat{0}}{ }_{\hat{0}} / \sqrt{\left|\operatorname{det} b_{\alpha \beta}\right|}$ which decay slower than $r^{-n}$, or at that rate. Similarly one sees from (8.10)(8.11) that only those terms in

$$
\Delta^{\hat{\alpha} \hat{\nu}}=\sqrt{\left|\operatorname{det} g_{\hat{\rho} \hat{\sigma}}\right|} g^{\hat{\alpha} \hat{\nu}}-b^{\hat{\alpha} \hat{\nu}}
$$

(compare (8.5)) which are $O\left(r^{-n}\right)$, or which are decaying slower, will give a non-vanishing contribution to the term involving the derivatives of $X$ in the integral (8.13). 
We will say that a metric is asymptotically Birmingham if there exists $\epsilon>0$ such that in the frame (8.6) it holds

$$
e^{\hat{\mu} \hat{\nu}}=O\left(r^{-n / 2-\epsilon}\right), \quad e_{\hat{\rho}}\left(e^{\hat{\mu} \hat{\nu}}\right)=O\left(r^{-n / 2-\epsilon}\right), \quad \operatorname{det}\left(e^{\hat{\mu} \hat{\nu}}\right)-1=O\left(r^{-n-\epsilon}\right) .
$$

We note that we have imposed the volume-element condition to guarantee convergence of mass integrals, see (8.16)-(8.17) below.

Recall that we only consider vector fields $X$ which are $b$-Killing vector fields, and therefore their tetrad components satisfy

$$
\left|X^{\hat{\nu}}\right|+\left|\stackrel{\circ}{\hat{\mu}}_{\hat{\mu}} X^{\hat{\nu}}\right| \leq C r .
$$

We claim that (8.14) guarantee a finite total energy in vacuum. Indeed, this follows from the standard integral identity (cf., e.g., [9]),

$$
\int_{\left\{x^{0}=0, r=R\right\}} \mathbb{U}^{\alpha \beta} d S_{\alpha \beta}=2 \int_{\left\{x^{0}=0, R_{0} \leq r \leq R\right\}} \stackrel{\circ}{\nabla}_{\beta} \mathbb{U}^{\alpha \beta} d S_{\alpha}+\int_{\left\{x^{0}=0, r=R_{0}\right\}} \mathbb{U}^{\alpha \beta} d S_{\alpha \beta},
$$

with

$$
\begin{aligned}
16 \pi & \stackrel{\circ}{\nabla}_{\beta} \mathbb{U}^{\alpha \beta}=\left(\sqrt{|\operatorname{det} g|} g^{\alpha \gamma}-\sqrt{|\operatorname{det} b|} b^{\alpha \gamma}\right) b_{\gamma \beta} X^{\beta} \\
& +\left(\stackrel{\mathscr{T}}{\alpha}^{\alpha}{ }_{\kappa}-\mathscr{T}^{\alpha}{ }_{\kappa}\right) X^{\kappa}+2 \Lambda(\sqrt{|\operatorname{det} b|}-\sqrt{|\operatorname{det} g|}) X^{\beta} \\
& +\sqrt{|\operatorname{det} b|}\left(Q^{\alpha}{ }_{\beta} X^{\beta}+Q^{\alpha}{ }_{\beta \gamma} \stackrel{\circ}{\nabla}^{\beta} X^{\gamma}\right)
\end{aligned}
$$

where $Q^{\alpha}{ }_{\beta}$ is a quadratic form in $e_{a}\left(e^{b c}\right)$, and $Q^{\alpha}{ }_{\beta \gamma}$ is bilinear in $e_{a}\left(e^{b c}\right)$ and $e^{a b}$, both with bounded coefficients. Finally,

$$
8 \pi \mathscr{T}_{\kappa}^{\lambda}:=\sqrt{|\operatorname{det} g|}\left(R_{\kappa}^{\lambda}-\frac{1}{2} g^{\alpha \beta} R_{\alpha \beta} \delta_{\kappa}^{\lambda}+\Lambda \delta_{\kappa}^{\lambda}\right)
$$

with $\check{\mathscr{T}}^{\lambda}{ }_{\kappa}$ defined as in (8.18) with $g$ replaced by $b$.

Passing with $R$ to infinity in (8.16), under (8.14) the right-hand side converges to a finite limit in vacuum, and one finds indeed that the resulting Hamiltonians are finite.

If the metric is not vacuum, the same argument applies if one moreover assumes that there exists $\epsilon>0$ such that

$$
\left|\mathscr{T}^{\mu}{ }_{\nu}-\stackrel{\circ}{\mathscr{T}}^{\mu}{ }_{\nu}\right| \leq C(1+r)^{-1-\epsilon} .
$$

We note that for the calculations of the boundary term the following, slightly weaker, conditions suffice:

$$
e^{\hat{\mu} \hat{\nu}}=o\left(r^{-n / 2}\right), \quad e_{\hat{\rho}}\left(e^{\hat{\mu} \hat{\nu}}\right)=o\left(r^{-n / 2}\right) .
$$

The boundary conditions (8.20) ensure that one needs to keep track only of those terms in $\mathbb{U}^{\hat{1} \hat{0}}$ which are linear in $e^{\hat{\mu} \hat{\nu}}$ and $e_{\hat{\rho}}\left(e^{\hat{\mu} \hat{\nu}}\right)$, when $\mathbb{U}^{\hat{1} \hat{0}}$ is Taylorexpanded around $b$. 
For example, if $g$ has the same leading order terms as a Birmingham metric (6.1)-(6.2) we find, writing $f_{0}$ for $\left.f\right|_{m=0}$, using (8.6)

$$
g^{\sharp}:=g^{\hat{\mu} \hat{\nu}} e_{\hat{\mu}} e_{\hat{\nu}}=-\frac{f_{0}}{f}\left(e_{\hat{0}}\right)^{2}+\frac{f}{f_{0}}\left(e_{\hat{1}}\right)^{2}+\sum_{\hat{A}=2}^{n-1}\left(e_{\hat{A}}\right)^{2},
$$

which yields

$$
\begin{gathered}
e^{\hat{0} \hat{0}}=-\frac{f_{0}}{f}+1=\frac{f-f_{0}}{f}=-\frac{2 m \ell^{2}}{r^{n}}\left(1+O\left(r^{-2}\right)\right), \\
e^{\hat{1} \hat{1}}=\frac{f_{0}-f}{f_{0}}=-\frac{2 m \ell^{2}}{r^{n}}\left(1+O\left(r^{-2}\right)\right), \\
e_{\hat{1}}\left(e^{\hat{0} \hat{0}}\right)=f_{0} \partial_{r}\left(\frac{f-f_{0}}{f_{0}}\right)=\frac{2 n m \ell}{r^{n}}\left(1+O\left(r^{-2}\right)\right), \\
e_{\hat{1}}\left(e^{\hat{1} \hat{1}}\right)=f_{0} \partial_{r}\left(\frac{f-f_{0}}{f_{0}}\right)=\frac{2 n m \ell}{r^{n}}\left(1+O\left(r^{-2}\right)\right),
\end{gathered}
$$

with the remaining $e^{\hat{\mu} \hat{\nu}}$ 's and $e_{\hat{\sigma}}\left(e^{\hat{\mu} \hat{\nu}}\right)$ 's vanishing, so that Equations (8.20) are satisfied for metrics with leading Birmingham asymptotics.

Rather generally, under (8.20) one obtains, using $b_{\hat{\mu} \hat{\nu}}=\operatorname{diag}(-1,+1, \cdots,+1)$,

$$
\begin{aligned}
& g_{\hat{\mu} \hat{\nu}}=b_{\hat{\mu} \hat{\nu}}-b_{\hat{\mu} \hat{\alpha}} b_{\hat{\nu} \hat{\beta}} e^{\hat{\alpha} \hat{\beta}}+o\left(r^{-n}\right) \text {, } \\
& \sqrt{\left|\operatorname{det} g_{\mu \nu}\right|}=\sqrt{\left|\operatorname{det} b_{\mu \nu}\right|}\left(1+\frac{1}{2}\left(e^{\hat{0} \hat{0}}-e^{\hat{1} \hat{1}}-b_{\hat{A} \hat{B}} e^{\hat{A} \hat{B}}\right)+o\left(r^{-n}\right)\right) \text {, } \\
& \frac{16 \pi \mathbb{U}_{\hat{0}}^{\hat{1} \hat{0}}}{\sqrt{\left|\operatorname{det} b_{\alpha \beta}\right|}}=\frac{2 \sqrt{\left|\operatorname{det} b_{\mu \nu}\right|}}{\sqrt{\left|\operatorname{det} g_{\rho \sigma}\right|}} g_{\hat{0} \hat{\gamma}}\left(e^{2} g^{\hat{\gamma}[\hat{1}} g^{\hat{0}] \hat{\kappa}}\right)_{; \hat{\kappa}} \\
& =4 g_{\hat{0} \hat{\gamma}} g^{\hat{\gamma}[\hat{1}} g^{\hat{0}] \hat{\kappa}} e_{; \hat{\kappa}}+2 e g_{\hat{0} \hat{\gamma}}\left(g^{\hat{\gamma}[\hat{1}} g^{\hat{0}] \hat{\kappa}}\right)_{; \hat{\kappa}} \\
& =-2 g^{1 \hat{\kappa}} e_{; \hat{\kappa}}+2 e g_{\hat{0} \hat{\gamma}}\left(\left(b^{\hat{\gamma}[\hat{1}}+e^{\hat{\gamma}[\hat{1}}\right)\left(b^{\hat{0}] \hat{\kappa}}+e^{\hat{0}] \hat{\kappa}}\right)\right)_{; \hat{\kappa}} \\
& =-2 e_{; \hat{1}}+2 b_{\hat{0} \hat{\gamma}}\left(b^{\hat{\gamma}[\hat{1}} b^{\hat{0}] \hat{\kappa}}+b^{\hat{\gamma}[\hat{1}} e^{\hat{0}] \hat{\kappa}}+e^{\hat{\gamma}[\hat{1}} b^{\hat{0}] \hat{\kappa}}+e^{\hat{\gamma}[\hat{1}} e^{0]}\right)_{; \hat{\kappa}}+o\left(r^{-n}\right) \\
& =-2 e_{; \hat{1}}+2 b_{\hat{0} \hat{0}}\left(b^{\hat{0}[\hat{1}} e^{\hat{0}] \hat{\kappa}}+e^{\hat{0}[\hat{1}} b^{\hat{0}] \hat{\kappa}}\right)_{; \hat{\kappa}}+o\left(r^{-n}\right) \\
& =-2 e_{; \hat{1}}-\left(-b^{\hat{0} \hat{0}} e^{\hat{1} \hat{\kappa}}+e^{\hat{0} \hat{1}} b^{\hat{0} \hat{\kappa}}-e^{\hat{0} \hat{0}} b^{\hat{1} \hat{\kappa}}\right)_{; \hat{\kappa}}+o\left(r^{-n}\right) \\
& =-2 e_{; \hat{1}}-e_{; \hat{1} \hat{\kappa}}+e_{; \hat{0} \hat{1}}+e_{; \hat{0}}^{\hat{0} \hat{0}}+o\left(r^{-n}\right) \text {. }
\end{aligned}
$$

This can be further rewritten as

$$
\begin{aligned}
\frac{16 \pi \mathbb{U}^{\hat{1} \hat{0}} \hat{0}}{\sqrt{\left|\operatorname{det} b_{\alpha \beta}\right|}}= & -2 e_{; \hat{1}}-e_{; \hat{1} \hat{1}}-e_{; \hat{1} \hat{A}}+e^{\hat{0} \hat{0}} ; \hat{1} \\
= & e_{\hat{1}}\left(b_{\hat{A} \hat{B}} e^{\hat{A} \hat{B}}\right)+\omega_{\hat{A} \hat{1} \hat{B}} e^{\hat{A} \hat{B}}-\omega^{\hat{A}}{ }_{\hat{1} \hat{A}} e^{\hat{1} \hat{1}}-\frac{1}{r} \dot{\mathcal{D}}_{\hat{A}} e^{\hat{1} \hat{A}}+o\left(r^{-n}\right) \\
= & e_{\hat{1}}\left(b_{\hat{A} \hat{B}} e^{\hat{A} \hat{B}}\right)+\sqrt{\frac{\beta}{r^{2}}+\frac{1}{\ell^{2}}}\left(b_{\hat{A} \hat{B}} e^{\hat{A} \hat{B}}-(n-1) e^{\hat{1} \hat{1}}\right) \\
& -\frac{1}{r} \mathcal{D}_{\hat{A}} e^{\hat{1} \hat{A}}+o\left(r^{-n}\right),
\end{aligned}
$$

Here $\stackrel{\circ}{\mathcal{D}}_{\hat{A}}$ denotes the covariant derivative on $(\stackrel{\circ}{\mathscr{N}}, \stackrel{\circ}{h})$, with $e^{\hat{1} \hat{A}}$ being understood

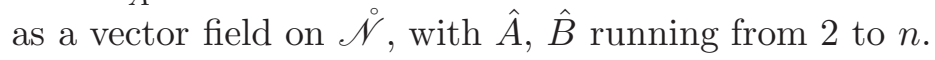


We also have

$$
\begin{aligned}
\frac{1}{8 \pi} \Delta^{\hat{\alpha}[\hat{1}}\left(X^{\hat{0}]}\right)_{; \hat{\alpha}} & =\frac{1}{16 \pi}\left(\Delta^{\hat{1} \hat{1}}-\Delta^{\hat{0} \hat{0}}\right)\left(X^{\hat{0}}\right)_{; \hat{1}}=\frac{r}{16 \pi \ell^{2}}\left(\Delta^{\hat{1} \hat{1}}-\Delta^{\hat{0} \hat{0}}\right) \\
& =-\frac{r}{16 \pi \ell^{2}} b_{\hat{A} \hat{B}} e^{\hat{A} \hat{B}}+o\left(r^{-n}\right) .
\end{aligned}
$$

Inserting all this into (8.13) one is finally led to the following simple expression for the Hamiltonian mass of asymptotically Birmingham metrics:

$$
\begin{gathered}
m_{\mathrm{Ham}}=\lim _{R \rightarrow \infty} \frac{r^{n}}{16 \pi} \int_{\mathscr{S} \cap\{r=R\}}\left[\left(\frac{1}{\ell^{2}}+\frac{\beta}{r^{2}}\right)\left(\frac{r \partial\left(b_{\hat{A} \hat{B}} e^{\hat{A} \hat{B}}\right)}{\partial r}-(n-1) e^{\hat{\hat{1}} \hat{1}}\right)\right. \\
\left.+\frac{\beta}{r^{2}} b_{\hat{A} \hat{B}} e^{\hat{A} \hat{B}}\right] d^{n-1} \mu_{h},
\end{gathered}
$$

In space-time dimension $n+1=4$ this simplifies to the expression given in [19]:

$$
m_{\mathrm{Ham}}=\lim _{R \rightarrow \infty} \frac{R^{3}}{16 \pi \ell^{2}} \int_{\mathscr{S} \cap\{r=R\}}\left[r \frac{\partial e^{\hat{2} \hat{2}}}{\partial r}+r \frac{\partial e^{\hat{3} \hat{3}}}{\partial r}-2 e^{\hat{1} \hat{1}}\right] d^{n-1} \mu_{h} .
$$

If in addition to (8.14) we assume that

$$
e^{\hat{\mu} \hat{\nu}}=O\left(r^{-n}\right), \quad e_{\hat{\rho}}\left(e^{\hat{\mu} \hat{\nu}}\right)=O\left(r^{-n}\right)
$$

(this is actually the fall-off rate for Birmingham metrics), (8.28) can be rewritten in a form similar to (8.29) in higher dimensions as well:

$m_{\mathrm{Ham}}=\lim _{R \rightarrow \infty} \frac{r^{n}}{16 \pi \ell^{2}} \int_{\mathscr{S} \cap\{r=R\}}\left[\left(\frac{r \partial\left(b_{\hat{A} \hat{B}} e^{\hat{A} \hat{B}}\right)}{\partial r}-(n-1) e^{\hat{1} \hat{1}}\right)\right] d^{n-1} \mu_{h}$.

As an example, if $g$ is the $3+1$-dimensional Birmingham metric (6.1), we find

$$
m_{\mathrm{Ham}}=\frac{\mu_{\grave{h}}(\stackrel{\circ}{\mathscr{N}}) m}{4 \pi},
$$

where

$$
\mu_{h}(\stackrel{\circ}{\mathscr{N}}):=\int_{\mathscr{N}} d^{n-1} \mu_{h}^{\circ}
$$

We conclude that the Hamiltonian mass is proportional to $m$, with the same proportionality factor as the characteristic mass of null hypersurfaces asymptotic to level sets of $t$, see (7.5):

$$
m_{\mathrm{TB}}=m_{\mathrm{Ham}} .
$$

When $\stackrel{\circ}{\mathscr{N}}=T^{2}$ (equivalently, $\beta=0$ ) with area normalized to $4 \pi$ we obtain $m_{\text {Ham }}=m$. For $\beta= \pm 1$ it follows from the Gauss-Bonnet theorem that $\mu_{\check{h}}(\stackrel{\mathscr{N}}{)})=4 \pi\left|1-g_{\infty}\right|$, where $g_{\infty}$ is the genus of $\mathscr{\mathscr { N }}$, hence

$$
m_{\mathrm{Ham}}=\left|1-g_{\infty}\right| m .
$$

One recovers $m_{\text {Ham }}=m$ for $\stackrel{\circ}{\mathscr{N}}=S^{2}$, but this will be true only up to a positive proportionality factor for $\mathscr{N}$ 's of higher genus. 


\subsection{Asymptotically HM-type metrics}

The aim of this section is to derive a formula analogous to (8.31) for metrics with Horowitz-Myers-type asymptotics. For this consider, as before, the background metric

$$
b:=-e_{\hat{0}} \otimes e_{\hat{0}}+e_{\hat{1}} \otimes e_{\hat{1}}+\sum_{A=2}^{n} e_{\hat{A}} \otimes e_{\hat{A}},
$$

where now instead of (8.6) we set

$$
e_{\hat{0}}=\frac{\ell}{r} \partial_{0}, \quad e_{\hat{1}}=\sqrt{\beta+\frac{r^{2}}{\ell^{2}}} \partial_{r}, \quad e_{\hat{A}}=\frac{1}{r} \iota_{\hat{A}}, \quad e_{\hat{n}}=\frac{1}{\ell \lambda \sqrt{\beta+\frac{r^{2}}{\ell^{2}}}} \partial_{\alpha} .
$$

Here $\iota_{\hat{A}}$ is an ON frame for the metric $\breve{h}$ as in the first line of (7.10), and in this section we let

$$
A, B \in\{2,3, \ldots, n-1\}
$$

similarly for hatted indices.

A metric $g$ will be said to be asymptotically $H M$ along $\mathscr{S}$, or simply asymptotically $H M$, if there exists a coordinate system $\left(t, r, x^{A}\right)$ and $\epsilon>0$ such that at $\mathscr{S}:=\{t=0\}$ we have

$$
\frac{\operatorname{det} g_{\mu \nu}}{\operatorname{det} b_{\mu \nu}}=1+O\left(r^{-n-\epsilon}\right),
$$

and if the frame components of $g$ with respect to the frame (8.36) satisfy

$$
e^{\hat{\alpha} \hat{\beta}}:=g^{\hat{\alpha} \hat{\beta}}-b^{\hat{\alpha} \hat{\beta}}=O\left(r^{-n / 2-\epsilon}\right), \quad e_{\hat{\mu}}\left(e^{\hat{\alpha} \hat{\beta}}\right)=O\left(r^{-n / 2-\epsilon}\right) .
$$

This is formally the same as (8.14), but both the frame and the background metric are different. (As before, the volume element condition is added to guarantee convergence of mass integrals.)

The identity (8.16) shows as before that conditions (8.38)-(8.39) guarantee a finite Hamiltonian mass in vacuum. We expect that the arguments of [15] can be adapted to this case to show that the mass is independent of the freedom of choice of coordinates and frames satisfying our conditions above, but we have not attempted to check this.

Similarly to the Birmingham case, our calculations of the boundary integral will be done with (8.39) replaced by the slightly weaker conditions

$$
e^{\hat{\alpha} \hat{\beta}}=o\left(r^{-n / 2}\right), \quad e_{\hat{\mu}}\left(e^{\hat{\alpha} \hat{\beta}}\right)=o\left(r^{-n / 2}\right), \quad e=1+o\left(r^{-n / 2}\right) .
$$

The connection coefficients $\omega_{\hat{\mu} \hat{\nu} \hat{\rho}}$ of the background metric $b$ read

$$
\begin{gathered}
\omega_{\hat{0} \hat{1} \hat{0}}=-\frac{\sqrt{\beta+\frac{r^{2}}{\ell^{2}}}}{r}=-\sqrt{\frac{\beta}{r^{2}}+\frac{1}{\ell^{2}}}=-\frac{1}{\ell}+O\left(r^{-2}\right), \\
\omega_{\hat{1} \hat{A} \hat{B}}=-\frac{\sqrt{\beta+\frac{r^{2}}{\ell^{2}}}}{r} b_{\hat{A} \hat{B}}, \\
\omega_{\hat{1} \hat{n} \hat{n}}=-\frac{r}{\ell^{2} \sqrt{\beta+\frac{r^{2}}{\ell^{2}}}}=-\frac{1}{\sqrt{\beta \frac{\ell^{4}}{r^{2}}+\ell^{2}}}=-\frac{1}{\ell}+O\left(r^{-2}\right) .
\end{gathered}
$$


The remaining possibly non-vanishing connection coefficients, which are not obtained from the above by permutations of indices, are the $\omega_{\hat{A} \hat{B} \hat{C}}$ 's, with $A \neq$ $B$. As in the previous section, the exact values of the $\omega_{\hat{A} \hat{B} \hat{C}}$ 's are not needed in what follows. We further have,

$$
\begin{gathered}
X^{\hat{0}}=\frac{r}{\ell}, \\
e_{\hat{1}}\left(X^{\hat{0}}\right)=\left(X^{\hat{0}}\right)_{; \hat{1}}=-X_{\hat{0} ; \hat{1}}=X_{\hat{1} ; \hat{0}}=\frac{1}{\ell} \sqrt{\beta+\frac{r^{2}}{\ell^{2}}}=\frac{r}{\ell^{2}}+O\left(r^{-1}\right),
\end{gathered}
$$

where all the remaining $X^{\hat{\mu}}$ 's and $X_{\hat{\mu} ; \hat{\nu}}$ 's are zero.

Writing $f_{0}$ for $\left.f\right|_{m=0}$, from (8.6) we see that the HM-type metrics can be written as

$$
g^{\sharp}=-e_{\hat{0}} \otimes e_{\hat{0}}+\frac{f}{f_{0}} e_{\hat{1}} \otimes e_{\hat{1}}+\sum_{\hat{A}=2}^{n-1} e_{\hat{A}} \otimes e_{\hat{A}}+\frac{f_{0}}{f} e_{\hat{n}} \otimes e_{\hat{n}} .
$$

This leads to

$$
\begin{gathered}
e^{\hat{1} \hat{1}}=g^{\hat{1} \hat{1}}-b^{\hat{1} \hat{1}}=\frac{f}{f_{0}}-1=\frac{f-f_{0}}{f_{0}}=-\frac{2 m \ell^{2}}{r^{n}}\left(1+O\left(r^{-2}\right)\right), \\
e^{\hat{0} \hat{0}}=0=e^{\hat{A} \hat{B}}, \quad e^{\hat{\mu} \hat{\nu}}=0 \text { for } \mu \neq \nu \\
e^{\hat{n} \hat{n}}=\frac{f_{0}-f}{f}=\frac{2 m \ell^{2}}{r^{n}}\left(1+O\left(r^{-2}\right)\right)
\end{gathered}
$$

which satisfies the decay conditions set forth above.

Quite generally, for metrics satisfying (8.40) we find as before

$$
e=1+\frac{1}{2}(\underbrace{e^{\hat{0} \hat{0}}-e^{\hat{1} \hat{1}}-b_{\hat{A} \hat{B}} e^{\hat{A} \hat{B}}-e^{\hat{n} \hat{n}}})+o\left(r^{-n}\right),
$$

and note that (8.38) implies that the underbraced term is also $o\left(r^{-n}\right)$. Equation (8.25) still applies and, taking into account (8.37), gives

$$
\begin{aligned}
\frac{16 \pi \mathbb{U}^{\hat{1} \hat{0}}{ }_{\hat{0}}}{\sqrt{\left|\operatorname{det} b_{\alpha \beta}\right|}=} & -2 e_{; \hat{1}}-e_{; \hat{1} \hat{1}}-e_{; \hat{A}}^{\hat{1} \hat{A}}-e_{; \hat{n}}^{\hat{1} \hat{n}}+e_{; \hat{0} \hat{\hat{0}}}+o\left(r^{-n}\right) \\
= & e_{\hat{1}}\left(b_{\hat{A} \hat{B}} e^{\hat{A} \hat{B}}+e^{\hat{n} \hat{n}}\right)-e_{\hat{n}}\left(e^{\hat{1} \hat{n}}\right)+\omega_{\hat{A} \hat{1} \hat{B}} e^{\hat{A} \hat{B}}-\omega^{\hat{A}}{ }_{\hat{1} \hat{A}} e^{\hat{1} \hat{1}} \\
& \quad+\omega_{\hat{n} \hat{1} \hat{n}}\left(e^{\hat{n} \hat{n}}-e^{\hat{1} \hat{1}}\right)-\frac{1}{r} \check{\mathcal{D}}_{\hat{A}} e^{\hat{1} \hat{A}}+o\left(r^{-n}\right) \\
= & e_{\hat{1}}\left(b_{\hat{A} \hat{B}} e^{\hat{A} \hat{B}}+e^{\hat{n} \hat{n}}\right)-e_{\hat{n}}\left(e^{\hat{1} \hat{n}}\right)+\sqrt{\frac{\beta}{r^{2}}+\frac{1}{\ell^{2}}}\left(b_{\hat{A} \hat{B}} e^{\hat{A} \hat{B}}-(n-2) e^{\hat{1} \hat{1}}\right) \\
& +\left(\beta \frac{\ell^{4}}{r^{2}}+\ell^{2}\right)^{-1 / 2}\left(e^{\hat{n} \hat{n}}-e^{\hat{\hat{1}} \hat{1}}\right)-\frac{1}{r} \check{\mathcal{D}}_{\hat{A}} e^{\hat{1} \hat{A}}+o\left(r^{-n}\right) .
\end{aligned}
$$

Here $\check{\mathcal{D}}_{\hat{A}} e^{\hat{1} \hat{A}}$ is understood as the covariant divergence of the vector field $e^{\hat{1} \hat{A}} e_{\hat{A}}$ with respect to the metric $\check{h}$. 
Furthermore,

$$
\begin{aligned}
\frac{1}{8 \pi} \Delta^{\hat{\alpha}[\hat{1}}\left(X^{\hat{0}]}\right)_{; \hat{\alpha}} & =\frac{1}{16 \pi}\left(\Delta^{\hat{1} \hat{1}}-\Delta^{\hat{0} \hat{0}}\right)\left(X^{\hat{0}}\right)_{; \hat{1}} \\
& =\frac{1}{16 \pi \ell}\left(\Delta^{\hat{1} \hat{1}}-\Delta^{\hat{0} \hat{0}}\right) \sqrt{\beta+\frac{r^{2}}{\ell^{2}}+o\left(r^{-n}\right)} \\
& =-\frac{1}{16 \pi \ell}\left(b_{\hat{A} \hat{B}} e^{\hat{A} \hat{B}}+e^{\hat{n} \hat{n}}\right) \sqrt{\beta+\frac{r^{2}}{\ell^{2}}}+o\left(r^{-n}\right) .
\end{aligned}
$$

Inserting all the results into (8.13) we finally find the following expression for the Hamiltonian mass for asymptotically HM metrics, where we have used the fact that some terms integrate out to zero:

$$
\begin{aligned}
m_{\mathrm{Ham}} & =\lim _{R \rightarrow \infty} \frac{r^{n}}{16 \pi \ell^{2}} \int_{\mathscr{S} \cap\{r=R\}}\left[\frac{e^{\hat{n} \hat{n}}-e^{\hat{1} \hat{1}}}{\sqrt{\frac{\beta \ell^{2}}{r^{2}}+1}}\right. \\
& \left.+\sqrt{1+\frac{\beta \ell^{2}}{r^{2}}}\left(\frac{r \partial\left(b_{\hat{A} \hat{B}} e^{\hat{A} \hat{B}}+e^{\hat{n} \hat{n}}\right)}{\partial r}-(n-2) e^{\hat{1} \hat{1}}-e^{\hat{n} \hat{n}}\right)\right] d^{n-1} \mu_{\hat{h}},
\end{aligned}
$$

where $d^{n-1} \mu_{\hat{h}}$ is the measure element associated with the metric (7.15). In space-time dimension $n+1=4$ this coincides formally with (8.29)

$$
m_{\mathrm{Ham}}=\lim _{R \rightarrow \infty} \frac{r^{3}}{16 \pi \ell^{2}} \times \int_{\mathscr{S} \cap\{r=R\}}\left[\frac{r \partial\left(e^{\hat{2} \hat{2}}+e^{\hat{3} \hat{3}}\right)}{\partial r}-2 e^{\hat{\hat{1}} \hat{1}}\right] d^{n-1} \mu_{\hat{h}} .
$$

As an example, if $g$ is the $3+1$-dimensional Horowitz-Myers metric, we find

$$
m_{\mathrm{Ham}}=-\frac{\mu_{\hat{h}}(\stackrel{\circ}{\mathscr{N}}) m}{8 \pi}
$$

where

$$
\mu_{\hat{h}}(\stackrel{\mathscr{N}}{)}):=\lim _{R \rightarrow \infty} \int_{\mathscr{S} \cap\{r=R\}} d^{n-1} \mu_{\hat{h}}
$$

This coincides with what we found for the coordinate mass of Horowitz-Myers metrics, where however we had to restrict ourselves to the case $\beta=0$. We see that no such restriction arises for the Hamiltonian mass.

\subsection{Fefferman-Graham asymptotics with an ultrastatic confor- mal infinity}

In this section we assume that $n=3$ and $\Lambda<0$, unless explicitly indicated otherwise. We consider a vacuum space-time with a smooth conformal completion, thus both the background metric $b$ and $g$ have a Fefferman-Graham expansion as in (2.12) in a suitable coordinate system such that $x=0$ at $\mathscr{I}$ :

$$
\begin{aligned}
& b=x^{-2} \ell^{2}\left(d x^{2}+\tilde{b}_{a b}\left(x, x^{c}\right) d x^{a} d x^{b}\right), \\
& g=x^{-2} \ell^{2}\left(d x^{2}+\tilde{g}_{a b}\left(x, x^{c}\right) d x^{a} d x^{b}\right),
\end{aligned}
$$


where $\left(x^{a}\right)=\left(t, x^{A}\right)$, and with the coordinate components $\tilde{b}_{a b}$ asymptotic to $\tilde{g}_{a b}$ as $O\left(x^{3}\right)$. Here we have used the same compactifying factor

$$
\Omega=x / \ell
$$

to pass from $g$ to $\tilde{g}=\Omega^{2} g$ as from $b$ to $\tilde{b}=\Omega^{2} b$.

For simplicity we will assume an ultrastatic form of the conformal-boundary metric

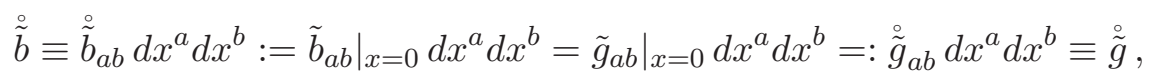

namely

$$
\tilde{b}_{0 A}\left(0, x^{c}\right)=0, \quad \partial_{a} \tilde{b}_{00}\left(0, x^{c}\right)=0, \quad \partial_{0} \tilde{b}_{A B}\left(0, x^{c}\right)=0
$$

(compare the discussion after (2.35)). Note that this is compatible both with asymptotically Birmingham and asymptotically Horowitz-Myers metrics. More general metrics and sections of $\mathscr{I}$ will be considered in future work.

Let $\left(\tilde{b}_{a b}\right)_{n}$ denote the coefficient of $x^{n}$ in a Taylor expansion of $\tilde{b}_{a b}$ at $x=$ 0 , similarly for $\left(\tilde{g}_{a b}\right)_{n}$. (The reader is warned that these coefficients do not translate as such to expansion coefficients in e.g. Bondi coordinates, as $r_{\mathrm{Bo}} \neq$ $1 / x$ in general even if $\ell=1$, see (8.78) below.) It follows from Section 2.2 that

$$
\left(\tilde{b}_{a b}\right)_{n}=\left(\tilde{g}_{a b}\right)_{n}, n \in\{0,1,2\} \text { and }\left(\tilde{b}_{a b}\right)_{1}=0 .
$$

In the calculations below we will assume that

$$
\left(\tilde{b}_{a b}\right)_{3}=0 \text {. }
$$

If this is not the case, in all the formulae below it suffices to replace $\left(\tilde{g}_{a b}\right)_{3}$ by $\left(\tilde{g}_{a b}\right)_{3}-\left(\tilde{b}_{a b}\right)_{3}$.

We wish to determine the characteristic mass of a null hypersurface asymptotic to a section of $\mathscr{I}$ with constant $x^{0}$, and compare it with the Hamiltonian mass $m_{\text {Ham }}$. Without loss of generality, after choosing a conformal gauge appropriately, we can assume that $\left.\tilde{b}_{A B}\right|_{x=0} d x^{A} d x^{B}$ has constant scalar curvature $\beta$. It then follows from $(2.15)$ that

$$
\left(\tilde{b}_{0 A}\right)_{2}=0 \quad \Longleftrightarrow \quad \tilde{b}_{0 A}=O\left(x^{3}\right)
$$

We pass now to the calculation of the Hamiltonian mass of $g$. Using $e^{11}=$ $0=e^{\hat{1} \hat{1}}$ and the first line of (8.26) (which applies here), we find quite generally in dimension $n$, without assuming (8.55),

$$
\frac{16 \pi \mathbb{U}^{\hat{1} \hat{0}} \hat{0}}{\sqrt{\left|\operatorname{det} b_{\alpha \beta}\right|}}=e_{\hat{1}}\left(b_{\hat{A} \hat{B}} e^{\hat{A} \hat{B}}\right)+\left(2 \omega^{\hat{0}} \hat{A} \hat{1}-\omega^{\hat{1}} \hat{0} \hat{A}\right) e^{\hat{0} \hat{A}}-\omega^{\hat{1}} \hat{A} \hat{B}^{\hat{A} \hat{B}}+o\left(x^{n}\right) \text {. }
$$

Returning to the three-dimensional ultrastatic case, we choose the $b$-orthonormal frame $e_{\hat{\alpha}}$ as

$$
e_{\hat{1}}=\frac{x}{\ell} \partial_{x}, \quad e_{\hat{0}}=\frac{x}{\ell \sqrt{\left|\tilde{b}_{00}\right|}} \partial_{0}, \quad e_{\hat{A}}=\frac{x}{\ell}\left(\psi_{\hat{A}}+O\left(x^{2}\right) \partial_{B}+O\left(x^{3}\right) \partial_{0}\right),
$$


where $\left\{\psi_{\hat{A}}\right\}_{\hat{A}=2, \ldots, n}$ is an ON frame for $\stackrel{\complement}{b}_{A B} d x^{A} d x^{B}$. Let us denote by $\theta^{\hat{\mu}}$ the coframe dual to $e_{\hat{\nu}}$, then

$$
\theta^{\hat{1}}=\frac{\ell}{x} d x, \quad \theta^{\hat{0}}=\frac{\ell \sqrt{\left|\tilde{b}_{00}\right|}}{x} d x^{0}+O\left(x^{2}\right) d x^{A}, \quad \theta^{\hat{A}}=\frac{\ell}{x}\left(\iota^{\hat{A}}+O\left(x^{2}\right) d x^{a}\right),
$$

where $\iota^{\hat{A}}$ is a coframe dual to $\psi_{\hat{A}}$. The components $g_{\hat{\mu} \hat{\nu}}$ of the metric $g$ with respect to this frame read

$$
\begin{gathered}
g_{\hat{1} \hat{1}}=1, \quad g_{\hat{1} \hat{a}}=0, \quad g_{\hat{0} \hat{0}}=-1+\frac{\left(\tilde{g}_{00}\right)_{3}}{\left|\tilde{b}_{00}\right|} x^{3}+o\left(x^{3}\right), \\
g_{\hat{0} \hat{A}}=\frac{\left(\tilde{g}_{0 \hat{A}}\right)_{3}}{\sqrt{\left|\tilde{b}_{00}\right|}} x^{3}+o\left(x^{3}\right), \quad g_{\hat{A} \hat{B}}=b_{\hat{A} \hat{B}}+\left(\tilde{g}_{\hat{A} \hat{B}}\right)_{3} x^{3}+o\left(x^{3}\right),
\end{gathered}
$$

where $\left(\tilde{g}_{0 \hat{A}}\right)_{3}$ denotes the $\iota$-component of $\left(\tilde{g}_{0 A}\right)_{3} d x^{A}$, as defined through the formula

$$
\left(\tilde{g}_{0 A}\right)_{3} d x^{A}=\left(\tilde{g}_{0 \hat{A}}\right)_{3} \iota^{\hat{A}} ; \text { similarly }\left(\tilde{g}_{A B}\right)_{3} d x^{A} d x^{B}=\left(\tilde{g}_{\hat{A} \hat{B}}\right)_{3} \iota^{\hat{A}} \iota^{\hat{B}} .
$$

This leads to

$$
\begin{gathered}
e^{\hat{A} \hat{B}}=-b^{\hat{A} \hat{C}} b^{\hat{B} \hat{D}}\left(\tilde{g}_{\hat{C} \hat{D}}\right)_{3} x^{3}+o\left(x^{3}\right)=-\left(\tilde{g}_{\hat{A} \hat{B}}\right)_{3} x^{3}+o\left(x^{3}\right), \\
e^{\hat{1} \hat{\mu}}=0, \quad e^{\hat{0} \hat{0}}=-\frac{\left(\tilde{g}_{00}\right)_{3}}{\left|\tilde{b}_{00}\right|} x^{3}+o\left(x^{3}\right), \quad e^{\hat{0} \hat{A}}=\frac{\left(\tilde{g}_{0 \hat{A}}\right)_{3}}{\sqrt{\left|\tilde{b}_{00}\right|}} x^{3}+o\left(x^{3}\right),
\end{gathered}
$$

where, of course, $b_{\hat{A} \hat{B}}=b^{\hat{A} \hat{B}}=\delta_{\hat{B}}^{\hat{A}}$. Note that the condition $e=1+o\left(x^{3}\right)$, which is equivalent to the $\tilde{\tilde{b}}_{a b}$-tracelessness of $\left(\tilde{g}_{a b}\right)_{3}$, reads

$$
-\frac{\left(\tilde{g}_{00}\right)_{3}}{\left|\tilde{b}_{00}\right|}+b^{\hat{A} \hat{B}}\left(\tilde{g}_{\hat{A} \hat{B}}\right)_{3} \equiv-\frac{\left(\tilde{g}_{00}\right)_{3}}{\left|\tilde{b}_{00}\right|}+\tilde{\tilde{b}}^{A B}\left(\tilde{g}_{A B}\right)_{3}=0 .
$$

Setting $\tilde{\tilde{b}}_{A B}:=\left.\tilde{b}_{A B}\right|_{x=0}$, and using $\omega^{\hat{\alpha}} \hat{\mu} \hat{\nu}=\theta^{\hat{\alpha}}\left(e_{\hat{\mu} ; \hat{\nu}}\right)$, we find

$$
\begin{gathered}
\Gamma_{A B}^{1}=\frac{\check{\check{b}}_{A B}}{x}+O\left(x^{2}\right), \omega^{\hat{1}}{ }_{\hat{A} \hat{B}}=\frac{1}{\ell} b_{\hat{A} \hat{B}}+O\left(x^{3}\right), \omega^{\hat{0}}{ }_{\hat{A} \hat{1}}=O\left(x^{2}\right), \\
\omega^{\hat{A}}{ }_{\hat{0} \hat{A}}=O\left(x^{4}\right), \quad \omega^{\omega_{\hat{0} \hat{A}}}=O\left(x^{4}\right) .
\end{gathered}
$$

This leads to the following rewriting of (8.59):

$$
\begin{aligned}
\frac{16 \pi \mathbb{U}^{\hat{1} \hat{0}} \hat{0}}{\sqrt{\left|\operatorname{det} b_{\alpha \beta}\right|}} & =\frac{x}{\ell} \partial_{x}\left(b_{\hat{A} \hat{B}} e^{\hat{A} \hat{B}}\right)-\frac{1}{\ell} b_{\hat{A} \hat{B}} e^{\hat{A} \hat{B}}+o\left(x^{3}\right) \\
& =-\frac{2}{\ell} b^{\hat{A} \hat{B}}\left(\tilde{g}_{\hat{A} \hat{B}}\right)_{3} x^{3}+o\left(x^{3}\right) .
\end{aligned}
$$

Next, we choose $X$ to be $\partial_{0}$, so that

$$
X=\partial_{0}=\frac{\ell \sqrt{\left|\tilde{b}_{00}\right|}}{x} e_{\hat{0}} \equiv X^{\hat{0}} e_{\hat{0}}, \quad X_{; \hat{1}}^{\hat{0}}=-\frac{\sqrt{\left|\tilde{b}_{00}\right|}}{\ell x}+O(x)=X_{; \hat{0}}^{\hat{1}},
$$




$$
\begin{gathered}
X_{; \hat{a}}^{\hat{1}}=O\left(x^{3}\right), \quad X_{; \hat{a}}^{\hat{0}}=O\left(x^{2}\right), \quad \Delta^{\hat{1} \hat{1}}=o\left(x^{3}\right), \quad \Delta^{\hat{1} \hat{a}}=0 \\
\Delta^{\hat{0} \hat{0}}=e^{\hat{0} \hat{0}}+o\left(x^{3}\right), \quad \Delta^{\hat{0} \hat{A}}=e^{\hat{0} \hat{A}}+o\left(x^{3}\right) \\
\frac{1}{8 \pi} \Delta_{; \hat{\alpha}}^{\hat{\alpha}[\hat{1}} X_{; \hat{0}}^{\hat{0}]}=\frac{\sqrt{\left|\tilde{b}_{00}\right|}}{16 \pi \ell x} e^{\hat{0} \hat{0}}+o\left(x^{2}\right)=-\frac{\sqrt{\left|\tilde{b}_{00}\right|}}{16 \pi} \times \frac{\left(\tilde{g}_{00}\right)_{3}}{\left|\tilde{b}_{00}\right| \ell} x^{2}+o\left(x^{2}\right) .
\end{gathered}
$$

Hence, using (8.1)-(8.3), for any hypersurface $\mathscr{S}$ intersecting $\mathscr{I}$ in a section $\left\{x^{0}=\right.$ const $\}$, after taking into account an overall minus sign because of the change of orientation when replacing $r$ by $x=\ell / r+\ldots$,

$$
\begin{aligned}
m_{\text {Ham }} & =-\lim _{x \rightarrow 0} \frac{\ell^{2}}{x^{2}} \int U^{\hat{1} \hat{0}} \sqrt{\operatorname{det} \stackrel{\tilde{b}}{A B}_{A}} d^{2} x \\
& =\frac{\ell \sqrt{\left|\tilde{b}_{00}\right|}}{16 \pi} \int\left(2 b^{\hat{A} \hat{B}}\left(\tilde{g}_{\hat{A} \hat{B}}\right)_{3}+\frac{\left(\tilde{g}_{00}\right)_{3}}{\left|\tilde{b}_{00}\right|}\right) \sqrt{\operatorname{det} \tilde{b}_{A B}} d^{2} x \\
& =\frac{3 \ell \sqrt{\left|\tilde{b}_{00}\right|}}{16 \pi} \int b^{\hat{A} \hat{B}}\left(\tilde{g}_{\hat{A} \hat{B}}\right)_{3} \sqrt{\operatorname{det} \tilde{\tilde{b}}_{A B}} d^{2} x
\end{aligned}
$$

It is clearly convenient to normalise the asymptotic time coordinate $x^{0}$ so that (compare (2.17))

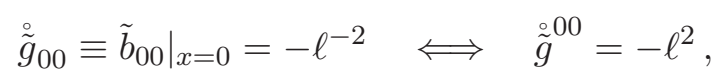

leading finally to

$$
m_{\mathrm{Ham}}=\frac{3}{16 \pi} \int b^{\hat{A} \hat{B}}\left(\tilde{g}_{\hat{A} \hat{B}}\right)_{3} \sqrt{\operatorname{det} \tilde{\tilde{b}}_{A B}} d^{2} x
$$

Note that this coincides formally with both (8.31) and (8.49), but it was not a priori clear to us that it should.

We wish to compare (8.74) with the characteristic mass as defined by (5.1)(5.2). For this, we need to determine the mass aspect function $M$ of (5.2). If the zero-level set of $u_{\mathrm{Bo}}$ is asymptotic to the zero-level set of $t$, an asymptotic expansion of the solutions of the equations which determine the Bondi coordinates shows that

$$
t=u_{\mathrm{Bo}}-\ell x-\frac{1}{6 \ell^{3}}\left(\tilde{g}_{00}\right)_{2} x^{3}+O\left(x^{4}\right), \quad x^{A}=x_{\mathrm{Bo}}^{A}+\frac{1}{3} \ell\left(\tilde{b}_{0 A}\right)_{2} x^{3}+O\left(x^{4}\right) .
$$

The above solution is obtained after imposing the condition that $u$ is a retarded null coordinate, hence $t$ is an increasing function of $r$ at fixed $u$, hence decreasing in $x$ at fixed $u$.

Changing coordinates, one finds

$$
g_{u_{\mathrm{Bo}} u_{\mathrm{Bo}}}=x^{-2}\left(-1+\ell^{2}\left(\tilde{g}_{00}\right)_{2} x^{2}+\ell^{2}\left(\tilde{g}_{00}\right)_{3} x^{3}+O\left(x^{4}\right)\right) .
$$

It remains to replace $x$ by a Bondi coordinate, defined through the formula

$$
r_{\mathrm{Bo}}=\left(\frac{\operatorname{det} g_{A B}}{\operatorname{det} \tilde{b}_{A B}}\right)^{1 / 4} \text {. }
$$


A Mathematica calculation gives

$$
\frac{1}{x}=\frac{r_{\mathrm{Bo}}}{\ell}-\frac{\ell \tilde{b}^{A B}\left(\tilde{g}_{A B}\right)_{2}}{4 r_{\mathrm{Bo}}}-\frac{\ell^{3} \tilde{\tilde{b}}^{A B}\left(\tilde{g}_{A B}\right)_{3}}{4 r_{\mathrm{Bo}}^{2}}+O\left(r_{\mathrm{Bo}}^{-3}\right) .
$$

Inserting into (8.77), one finds that the mass aspect is

$$
M \equiv \frac{\left(g_{u_{\mathrm{Bo}} u_{\mathrm{Bo}}}\right)_{1}}{2}=\frac{1}{2}\left(\ell^{3}\left(\tilde{g}_{00}\right)_{3}+\frac{1}{2} b^{\hat{A} \hat{B}}\left(\tilde{g}_{\hat{A} \hat{B}}\right)_{3}\right)=\frac{3 \ell}{4} b^{\hat{A} \hat{B}}\left(\tilde{g}_{\hat{A} \hat{B}}\right)_{3},
$$

where we have used (8.66). Comparing (8.76) and (5.1), we conclude that

$$
m_{\mathrm{Ham}}=m_{\mathrm{TB}},
$$

as desired.

\section{Conclusions}

We have introduced a natural notion of total mass for characteristic hypersurfaces in space-times with non-vanishing cosmological constant. The mass is a natural generalisation of the Trautman-Bondi mass, as defined for $\Lambda=0$. We have proved a generalisation of the positivity identity of [17]. The identity introduces the renormalised volume as a new global quantity associated to characteristic initial data sets. In the simplest case of light-cones in vacuum this is the identity (5.58), which we rewrite as

$$
\begin{aligned}
m_{\mathrm{TB}} & -\frac{\Lambda}{8 \pi}\left(\frac{1}{12} \int_{\mathscr{N}} \tau_{2}\left(\frac{\tau_{2}^{2}}{2}-\frac{|\sigma|_{4}^{2}}{3}\right) d \mu_{\grave{h}}+V_{\mathrm{ren}}\right) \\
& =\frac{1}{16 \pi} \int_{0}^{\infty} \int_{\mathscr{N}}\left(\frac{1}{2}|\xi|^{2}+|\sigma|^{2} \mathrm{e}^{\int_{r}^{\infty} \frac{\tilde{r} \tau-2}{2 \tilde{r}} d \tilde{r}}\right) d \mu_{\check{g}} d r .
\end{aligned}
$$

The left-hand side involves the renormalised volume together with objects which can be determined by looking at the asymptotic behaviour of the fields. This provides a new global positivity statment, proving indeed that the left-hand side of (9.1) is positive. It follows from [6] that the left-hand side vanishes if and only if the space-time is de Sitter or anti-de Sitter to the future of the light-cone.

The balance formula (9.1) raises the question of the right definition of mass when $\Lambda \neq 0$. Recall that we used (5.1)-(5.2) to define $m_{\mathrm{TB}}$ :

$$
m_{\mathrm{TB}}=\frac{1}{8 \pi} \int_{\mathscr{N}}\left(\bar{g}_{00}^{\mathrm{Bo}}\right)_{1} d \mu_{h} .
$$

A first naive idea would be to define instead the left-hand side of (9.1) as the mass, obtaining positivity as a corollary of (9.1). But the calculations in Appendix A strongly suggest that a splitting of the left-hand side of (9.1) in a renormalised-volume contribution and a mass contribution is meaningful.

The next idea would be to define the characteristic mass as

$$
m_{c}=m_{\mathrm{TB}}-\frac{\Lambda}{8 \pi}\left(\frac{1}{12} \int_{\mathscr{\mathfrak { N }}} \tau_{2}\left(\frac{\tau_{2}^{2}}{2}-\frac{|\sigma|_{4}^{2}}{3}\right) d \mu_{h}\right),
$$


leading to the more elegant identity:

$$
m_{c}-\frac{\Lambda}{8 \pi} V_{\text {ren }}=\frac{1}{16 \pi} \int_{0}^{\infty} \int_{\mathscr{r}}\left(\frac{1}{2}|\xi|^{2}+|\sigma|^{2} \mathrm{e}^{\int_{r}^{\infty} \frac{\tilde{r} \tau-2}{2 \tilde{r}} d \tilde{r}}\right) d \mu_{\check{g}} d r .
$$

Alternatively, one could add an integral expression involving $\tau_{2}$ and $|\sigma|_{4}$ to the definition of $m_{\mathrm{TB}}$, adjusting (9.1) accordingly. Recall that (9.2) is equivalent to (5.11), which for a smooth conformal completion reads

$$
m_{\mathrm{TB}}=\frac{1}{16 \pi} \int_{\mathscr{N}}\left(\zeta^{\mathrm{Bo}}\right)_{2} d \mu_{h} .
$$

In the asymptotically flat case and with spherical cross-sections of $\mathscr{I}$, the gaugeinvariant version of this formula is [17]

$$
\frac{1}{16 \pi} \int_{\mathscr{i}}\left(\zeta_{2}+\tau_{2}\right) d \mu_{h}
$$

and one could use this formula as a definition of characteristic mass. (Whether or not, and in which sense, this is gauge-invariant when $\Lambda \neq 0$ remains to be seen). Recall that we have seen (cf. (5.31) in vacuum and with a smooth conformal completion) that (9.5) translates instead into

$$
m_{\mathrm{TB}}=\frac{1}{16 \pi} \int_{\mathscr{\mathscr { N }}}\left(\zeta_{2}+\tau_{2}\right) d \mu_{h}+\frac{\Lambda}{16 \pi} \int_{\mathscr{\mathscr { N }}} \tau_{2}|\sigma|_{4}^{2} d \mu_{h}^{\circ}
$$

(note that the multiplicative factor $\stackrel{\circ}{R} / 2$ in front of $\tau_{2}$ in our formula equals 1 for a sphere), when $\Lambda \neq 0$ and an affine parameter $r$ is used.

As seen in Appendix A, we have $\tau_{2} \neq 0$ for asymptotically Horowitz-Myers metrics, which suggests strongly that using $\tau_{2}^{3}$-terms to redefine the mass is not a good idea. Whether or not adding some $\tau_{2}|\sigma|_{4}^{2}$-terms is meaningful requires further analysis. We plan to return to this question in the future.

Yet another alternative is to define the renormalized volume as the whole expression in brackets at the left-hand side of (9.1),

$$
V_{\text {ren }} \mapsto \widetilde{V}_{\text {ren }}:=V_{\text {ren }}+\frac{1}{12} \int_{\mathscr{\mathscr { N }}} \tau_{2}\left(\frac{\tau_{2}^{2}}{2}-\frac{|\sigma|_{4}^{2}}{3}\right) d \mu_{h}
$$

leading similarly to a nicer identity:

$$
m_{\mathrm{TB}}-\frac{\Lambda}{8 \pi} \widetilde{V}_{\mathrm{ren}}=\frac{1}{16 \pi} \int_{0}^{\infty} \int_{\mathscr{\mathscr { r }}}\left(\frac{1}{2}|\xi|^{2}+|\sigma|^{2} \mathrm{e}^{\int_{r}^{\infty} \frac{\tilde{r} \tau-2}{2 \tilde{r}} d \tilde{r}}\right) d \mu_{\check{g}} d r .
$$

Possibly, a mixture of the above will provide the most meaningful definitions.

Incidentally, $\widetilde{V}_{\text {ren }}$ can be obtained by replacing $r$ in the original definition of $V(r)$, where $r$ is the affine coordinate normalised as before, by

$$
r=\tilde{r}+\frac{\tau_{2}}{2}+\frac{a}{\tilde{r}^{2}}-\frac{2 \tau_{2}|\sigma|_{4}^{2}}{9 \tilde{r}^{2}},
$$

where $a$ is any function of the angular coordinates. In other words, set

$$
\widetilde{V}(\tilde{r}):=V\left(\tilde{r}+\frac{\tau_{2}}{2}+\frac{a}{\tilde{r}^{2}}-\frac{2 \tau_{2}|\sigma|_{4}^{2}}{9 \tilde{r}^{2}}\right) .
$$


Then $\widetilde{V}_{\text {ren }}$ is the limit, as $\tilde{r}$ goes to infinity, of $\tilde{V}(\tilde{r})$ minus the sum of the terms with positive powers of $\tilde{r}$ and the $\ln \tilde{r}$ term. In fact, a change of variables of the form

$$
r=\tilde{r}+\frac{\tau_{2}}{2}+\frac{|\sigma|_{4}^{2}}{2 \tilde{r}}-\frac{2 \tau_{2}|\sigma|_{4}^{2}}{9 \tilde{r}^{2}}+\frac{\tau_{2}|\sigma|_{4}^{2}}{3 \tilde{r}^{2}} \ln \tilde{r}+o\left(\tilde{r}^{-2}\right)
$$

gives

$$
V(r)=\frac{4 \pi}{3} \tilde{r}^{3}+V_{\text {ren }}+o\left(\tilde{r}^{-1}\right) .
$$

One wonders about the nature of (9.11). The naive guess would be that $\tilde{r}$ is the Bondi coordinate. However, in our case we have (in vacuum, but allowing $\left.|\sigma|_{5} \neq 0\right)$

$$
r_{\mathrm{Bo}}=r-\frac{\tau_{2}}{2}+\frac{|\sigma|_{4}^{2}}{4 r}+\frac{1}{6} \frac{|\sigma|_{5}^{2}-\frac{1}{4}|\sigma|_{4}^{2} \tau_{2}}{r^{2}}+O\left(r^{-3}\right),
$$

with inverse transformation

$$
r=r_{\mathrm{Bo}}+\frac{\tau_{2}}{2}-\frac{|\sigma|_{4}^{2}}{4 r_{\mathrm{Bo}}}+\frac{|\sigma|_{4}^{2} \tau_{2}-|\sigma|_{5}^{2}}{6 r_{\mathrm{Bo}}^{2}}+O\left(r_{\mathrm{Bo}}^{-3}\right)
$$

We see that $\tilde{r}$ coincides with the Bondi coordinate at order zero, but differs at the next order.

Which definition is most relevant, or indeed whether there exists a most relevant definition at all, requires further studies.

In any case, we have shown in some well understood general cases with $\Lambda \leq 0$, as well as on specific examples, that the characteristic mass $m_{\mathrm{TB}}$ defined by (9.7) coincides with previously accepted definitions of mass.

We emphasise that both the definition of mass and the balance formula (9.1) have a clear, geometric and gauge-independent, meaning; compare Remark 5.1.

\section{A Null geometry of Horowitz-Myers metrics and the balance equation}

The mass identity (5.56) can be viewed as a balance formula. It is instructive to work-out the contribution of each of the terms appearing there to the total mass for the Horowitz-Myers metrics. For this we need to derive the asymptotics both for small and large $r$ of the fields appearing there. We consider the metric (6.8) with $f$ given by (6.9) and $\beta=0$ in space-dimension equal to three.

Choosing $s\left(r_{0}\right)=0$, where $r_{0}$ is the largest zero of $f$, from (7.22) we obtain a small- $r$ expansion, for $r \geq r_{0}$ :

$$
s=\frac{2 r_{0}}{\ell \sqrt{f^{\prime}\left(r_{0}\right)}} \sqrt{r-r_{0}}+O\left(\left(r-r_{0}\right)^{\frac{3}{2}}\right) \text { for } r-r_{0}>0 \text { small },
$$

where we have assumed that $f^{\prime}\left(r_{0}\right) \neq 0$. This implies, for small $s$,

$$
\begin{aligned}
r-r_{0} & =\frac{\ell^{2} f^{\prime}\left(r_{0}\right)}{4 r_{0}^{2}} s^{2}+O\left(s^{4}\right), \\
f(r) & =\frac{\left(\ell f^{\prime}\left(r_{0}\right)\right)^{2}}{4 r_{0}^{2}} s^{2}+O\left(s^{4}\right) .
\end{aligned}
$$


Recall that the large- $s$ behaviour of $r$ has been derived in (7.24)-(7.25).

As in Section 3, we denote by $\check{g}$ the metric induced by $g$ on the level sets of $u$ and $r$ :

$$
\check{g}=f(r) \ell^{2} \lambda^{2} d \alpha^{2}+r^{2} d \varphi^{2} .
$$

Let $x^{A}$ denote the coordinates $\alpha$ and $\varphi$. In the affine parameterisation and in the region where $f$ is non-negative it holds that ${ }^{8}$

$$
\begin{aligned}
\sqrt{\operatorname{det} \check{g}_{A B}} & =r \ell \lambda \sqrt{f(r)}=\lambda \frac{\ell^{2} f^{\prime}\left(r_{0}\right)}{2}\left(s+O\left(s^{3}\right)\right) \quad \text { for small } s \\
\nu_{A} & =0 \\
\xi_{A} & =0 \\
\chi & :=\frac{1}{2} \partial_{s} \check{g}=\frac{\ell \sqrt{f(r)}}{2 r} \partial_{r}\left(f(r) \lambda^{2} \ell^{2} d \alpha^{2}+r^{2} d \varphi^{2}\right) \\
& =\ell \sqrt{f(r)}\left(\frac{\partial_{r} f(r)}{2 r} \lambda^{2} \ell^{2} d \alpha^{2}+d \varphi^{2}\right) \\
& \approx \frac{\ell^{2} f^{\prime}\left(r_{0}\right) s+O\left(s^{3}\right)}{2 r_{0}}\left(\frac{f^{\prime}\left(r_{0}\right)}{2 r_{0}} \lambda^{2} \ell^{2} d \alpha^{2}+d \varphi^{2}\right) \text { for small } s, \quad \text { for small } s \\
\tau & :=g^{A B} \chi_{A B}=\ell\left(\frac{\partial_{r} f(r)}{2 r \sqrt{f(r)}}+r^{-2} \sqrt{f(r)}\right) \\
& =\left\{\begin{array}{l}
s^{-1}+O(s), \\
2 s^{-1}+2\left(s_{*}-r_{0}\right) s^{-2}+O\left(s^{-3}\right), \quad \text { for large } s
\end{array}\right.
\end{aligned}
$$

with $s_{*}=(2 m \ell)^{1 / 3} x_{*}$, where $x_{*}$ is given by (7.27). Further

$$
\begin{aligned}
& \sigma:=\quad \chi-\frac{1}{2} \tau g_{A B} d x^{A} d x^{B}=\ell \sqrt{f(r)}\left(\frac{\partial_{r} f(r)}{2 r} \lambda^{2} \ell^{2} d \alpha^{2}+d \varphi^{2}\right) \\
& -\frac{1}{2} \ell\left(\frac{\partial_{r} f(r)}{2 r \sqrt{f(r)}}+r^{-2} \sqrt{f(r)}\right)\left(f \lambda^{2} \ell^{2} d \alpha^{2}+r^{2} d \varphi^{2}\right) \\
& =\frac{\ell}{2}\left(\frac{\partial_{r} f(r)}{2 r \sqrt{f(r)}}-r^{-2} \sqrt{f(r)}\right)\left(f \lambda^{2} \ell^{2} d \alpha^{2}-r^{2} d \varphi^{2}\right), \\
& \sigma^{\sharp}:=g^{A C} \sigma_{C B} \partial_{A} \otimes d x^{B} \\
& =\frac{\ell}{2}\left(\frac{\partial_{r} f(r)}{2 r \sqrt{f(r)}}-r^{-2} \sqrt{f(r)}\right)\left(\partial_{\alpha} \otimes d \alpha-\partial_{\varphi} \otimes d \varphi\right) \\
& = \begin{cases}\frac{\left(1+O\left(s^{2}\right)\right)}{2 s}\left(\partial_{\alpha} \otimes d \alpha-\partial_{\varphi} \otimes d \varphi\right), & \text { for small } s ; \\
\frac{\left(3 \ell^{2} m+O\left(s^{-1}\right)\right)}{2 s^{4}}\left(\partial_{\alpha} \otimes d \alpha-\partial_{\varphi} \otimes d \varphi\right), & \text { for large } s,\end{cases} \\
& \left|\sigma^{2}\right|=2 \times \frac{\ell^{2}}{4}\left(\frac{\partial_{r} f(r)}{2 r \sqrt{f(r)}}-r^{-2} \sqrt{f(r)}\right)^{2} \\
& = \begin{cases}\frac{\left(1+O\left(s^{2}\right)\right)}{2 s^{2}}, & \text { for small } s ; \\
\frac{\left(9 \ell^{4} m^{2}+O\left(s^{-1}\right)\right)}{2 s^{8}}, & \text { for large } s,\end{cases}
\end{aligned}
$$

\footnotetext{
${ }^{8}$ We use the notation of [7], except that we denote here by $\check{g}$ the tensor field denoted by $\tilde{g}$ there.
} 


$$
g^{s s}=g^{\mu \nu} \frac{\partial s}{\partial x^{\mu}} \frac{\partial s}{\partial x^{\nu}}=g^{r r}\left(\frac{d s}{d r}\right)^{2}=f\left(\frac{r}{\ell \sqrt{f}}\right)^{2}=\frac{r^{2}}{\ell^{2}},
$$

We will also need the following objects from [7], denoting by $\check{\nabla}$ the derivative operator associated with $\check{g}$, when $\check{g}$ is viewed as a metric on the level sets of $u$ and $r$ :

$$
\begin{aligned}
\bar{\Gamma}^{1} & :=\nu^{0} \bar{g}^{A B} \check{\nabla}_{B} \nu_{A}-\frac{\partial_{1}\left(\nu_{0} \bar{g}^{11} \sqrt{\operatorname{det} \check{g}}\right)}{\nu_{0} \sqrt{\operatorname{det} \check{g}}}-\frac{1}{2} \nu^{0} \bar{g}^{A B} \overline{\partial_{0} g_{A B}}+\frac{1}{2} \nu^{0} \bar{g}^{11} \frac{d}{\partial_{0} g_{11}} \\
\nu_{0} & :=g_{u s}=g_{u r} \frac{d r}{d s}=-\frac{r}{\ell \sqrt{f(r)}} \frac{d r}{d s}=-\frac{r}{\ell \sqrt{f(r)}} \frac{\ell \sqrt{f(r)}}{r}=-1 \\
\nu^{0} & :=\frac{1}{\nu_{0}}=-1 \\
\bar{\Gamma}^{s} & =-\frac{\partial_{s}\left(\nu_{0} \bar{g}^{s s} \sqrt{\operatorname{det} \check{g}}\right)}{\nu_{0} \sqrt{\operatorname{det} \check{g}}}=-\tau \bar{g}^{s s}-\partial_{s} \bar{g}^{s s}
\end{aligned}
$$

From the definition of $\zeta$ (compare [7, Equations (10.33) and (10.36)], ${ }^{9}$ and note that $\kappa \equiv \bar{\Gamma}_{s s}^{s} \equiv \bar{\Gamma}_{11}^{1}$ vanishes in affine parameterisation) we have

$$
\begin{aligned}
\zeta & :=\left(2 \partial_{s}+2 \bar{\Gamma}_{s s}^{s}+\tau\right) \bar{g}^{s s}+2 \bar{\Gamma}^{s}=-\tau \bar{g}^{s s} \\
& =-\ell^{-1}\left(\frac{r \partial_{r} f(r)}{2 \sqrt{f(r)}}+\sqrt{f(r)}\right) \\
& =\left\{\begin{array}{cc}
-r_{0}^{2} \ell^{-2} s^{-1}+O(s), & \text { for small } s \\
-2 r \ell^{-2}-m r^{-2}+O\left(r^{-5}\right) & \text { for large } s . \\
=-2\left(s+r_{0}-s_{*}\right) \ell^{-2}-2 m s^{-2}+O\left(s^{-5}\right),
\end{array}\right.
\end{aligned}
$$

Recall that the vacuum Raychaudhuri equation with affine parameter $s$,

$$
\partial_{s} \tau+\frac{\tau^{2}}{2}+|\sigma|^{2}=0
$$

can be solved as

$$
\tau(s)=\frac{2}{s}-s^{-2} \Psi^{-1} \int_{0}^{s}|\sigma|^{2} \Psi s^{2} d s
$$

where

$$
\Psi(s, \psi, \varphi)=\exp \left(-\int_{s}^{\infty} \frac{\tilde{s} \tau(\tilde{s}, \psi, \varphi)-2}{2 \tilde{s}} d \tilde{s}\right) .
$$

As $s$ tends to zero, the integral in (A.26) approaches infinity as $\frac{1}{2} \ln s$, hence the weight-factor $\Psi$ behaves as a constant times $s^{-1 / 2}$. This, together with the $1 /\left(2 s^{2}\right)$-behaviour of $|\sigma|^{2}$ for small $s$, leads in (A.25) to the required $1 / s$ behaviour of $\tau$ for $s$ approaching zero.

An alternative derivation of (A.22) proceeds by solving directly (3.15):

$$
\left(\partial_{s}+\tau\right) \zeta=\frac{1}{2}|\xi|^{2}-\check{\nabla}_{A} \xi^{A}-\check{R}+8 \pi\left(\bar{g}^{A B} \bar{T}_{A B}-\bar{T}\right)+2 \Lambda .
$$

\footnotetext{
${ }^{9}$ Note a missing term $\tau \bar{g}^{11} / 2$ in the rightermost term of [7, Equation (10.36)], which however does not affect the formula we use.
} 
In the current case $(\mathrm{A} .27)$ reads

$$
\left(\frac{d r}{d s} \partial_{r}+\tau\right) \zeta=2 \Lambda
$$

It follows from (A.28) that

$$
\frac{d(\zeta \sqrt{\operatorname{det} \check{g}})}{d s}=2 \Lambda \sqrt{\operatorname{det} \check{g}} .
$$

Integrating in $s$, we find

$$
\begin{aligned}
(\zeta \sqrt{\operatorname{det} \check{g}})(s) & =\underbrace{\lim _{s \rightarrow 0}(\zeta \sqrt{\operatorname{det} \check{g}})(s)}_{-3 m \lambda}+2 \Lambda \int_{0}^{s} \sqrt{\operatorname{det} \check{g}} d s \\
& =\lambda\left(-3 m+\frac{2 \Lambda}{3}\left(r^{3}-r_{0}^{3}\right)\right) \\
& =\lambda\left(-3 m+\frac{2 \Lambda}{3}\left(r^{3}-2 m \ell^{2}\right)\right),
\end{aligned}
$$

which coincides indeed with (A.22):

$$
\zeta \sqrt{\operatorname{det} \check{g}}=\lambda\left(m-\frac{2 r^{3}}{\ell^{2}}\right)=\lambda\left(m+\frac{2 \Lambda r^{3}}{3}\right) .
$$

We are ready now to check the contribution of various terms to the mass identity (5.56) for the Horowitz-Myers metrics (6.13). For these metrics we have $T_{\mu \nu} \equiv 0 \equiv \stackrel{\circ}{R} \equiv \xi \equiv|\sigma|_{4}^{2} \equiv|\sigma|_{5}^{2}$ (compare Equation (A.17)), and from (7.14) and (5.56) we find

$$
-2 m \mu_{\hat{h}}(\stackrel{\mathscr{N}}{)})=16 \pi m_{\mathrm{TB}}=\lim _{s \rightarrow 0} \int_{\mathscr{\mathscr { N }}} \zeta d \mu_{\check{g}}+\frac{\Lambda}{12} \int_{\mathscr{\mathscr { N }}} \tau_{2}^{3} d \mu_{\hat{h}}+2 \Lambda V_{\mathrm{ren}} .
$$

Recall that $\check{g}$ is defined as the angular part of the metric on the light-cone,

$$
\check{g}=\bar{g}_{A B} d x^{A} d x^{B}=f \lambda^{2} \ell^{2} d \alpha^{2}+r^{2} d \varphi^{2} .
$$

and that the limiting metric $\hat{h}$ defined in (7.15) is

$$
\hat{h}=\lim _{r \rightarrow \infty} r^{-2} \check{g}=\lambda^{2} d \alpha^{2}+d \varphi^{2} .
$$

Keeping in mind that the measure associated with $\hat{h}$ is

$$
d \mu_{\hat{h}}=\lambda d \alpha d \varphi
$$

(A.5) and (A.23) lead to

$$
\begin{aligned}
\lim _{r \rightarrow r_{0}} \int_{\mathscr{N}} \zeta d \mu_{\check{g}} & =-\frac{r_{0}^{2}}{\ell^{2}} \times \frac{\ell^{2} f^{\prime}\left(r_{0}\right)}{2} \times \mu_{\hat{h}}(\stackrel{\mathscr{N}}{)}) \\
& =-\frac{r_{0}^{2}}{\ell^{2}} \times \frac{\ell^{2} f^{\prime}\left(r_{0}\right)}{2} \times\left(\frac{1}{2 \ell f^{\prime}\left(r_{0}\right)} \times(2 \pi)^{2}\right)
\end{aligned}
$$


Equation (7.20) gives

$$
f^{\prime}\left(r_{0}\right)=\frac{1}{r_{0}}\left(2 \frac{r_{0}^{2}}{\ell^{2}}+\frac{2 m}{r_{0}}\right)=\frac{6 m}{r_{0}^{2}}=\frac{3\left(2 m \ell^{2}\right)^{\frac{1}{3}}}{\ell^{2}} .
$$

We can thus rewrite (A.32) as

$$
\begin{aligned}
\lim _{r \rightarrow r_{0}} \int_{\mathscr{N}} \zeta d \mu_{\check{g}} & =-3 m \times \mu_{\hat{h}}(\stackrel{\mathscr{N}}{)}) \\
& =-3 m \times \frac{2 \pi^{2} \ell}{3\left(2 m \ell^{2}\right)^{\frac{1}{3}}}=-3 m \times \frac{2 \pi^{2}}{3}\left(\frac{\ell}{2 m}\right)^{\frac{1}{3}} .
\end{aligned}
$$

The relation $\Lambda=-3 / \ell^{2}$ and (A.31) give the balance formula

$$
2 m \ell^{2} \mu_{\hat{h}}\left(\stackrel{\mathscr{N}}{)}=3 m \ell^{2} \mu_{\hat{h}}(\stackrel{\mathscr{N}}{)})+6 V_{\text {ren }}+\frac{1}{4} \int_{\mathscr{N}} \tau_{2}^{3} d \mu_{\hat{h}} .\right.
$$

We note that (A.11) gives $\tau_{2}=2\left(s_{*}-r_{0}\right)$, and that $(7.26)$ can be rewritten as

$$
V_{\text {ren }}=-\frac{1}{6}\left(m \ell^{2} \mu_{\hat{h}}(\stackrel{\circ}{\mathscr{N}})+2 \int_{\mathscr{N}}\left(s_{*}-r_{0}\right)^{3} d \mu_{\hat{h}}\right),
$$

in agreement with (A.35).

\section{References}

[1] L.F. Abbott and S. Deser, Stability of gravity with a cosmological constant, Nucl. Phys. B195 (1982), 76-96.

[2] A. Ashtekar, B. Bonga, and A. Kesavan, Asymptotics with a positive cosmological constant: I. Basic framework, Class. Quantum Grav. 32 (2015), 025004, 41 pp., arXiv:1409.3816 [gr-qc]. MR 3291776

[3] _ Asymptotics with a positive cosmological constant: II. Linear fields on de Sitter space-time, Phys. Rev. D92 (2015), 044011, arXiv:1506.06152 [gr-qc].

[4] D. Birmingham, Topological black holes in anti-de Sitter space, Class. Quantum Grav. 16 (1999), 1197-1205, arXiv:hep-th/9808032. MR MR1696149 (2000c:83062)

[5] H. Bondi, M.G.J. van der Burg, and A.W.K. Metzner, Gravitational waves in general relativity VII: Waves from axi-symmetric isolated systems, Proc. Roy. Soc. London A 269 (1962), 21-52. MR MR0147276 (26 \#4793)

[6] Y. Choquet-Bruhat, P.T. Chruściel, and J.M. Martín-García, The lightcone theorem, Class. Quantum Grav. 26 (2009), 135011 (22 pp), arXiv:0905.2133 [gr-qc]. MR 2515694 (2010g:53131)

[7] — The Cauchy problem on a characteristic cone for the Einstein equations in arbitrary dimensions, Ann. H. Poincaré 12 (2011), 419-482, arXiv:1006.4467 [gr-qc]. MR 2785136 
[8] P.T. Chruściel, Lectures on energy in general relativity, http://homepage. univie.ac.at/piotr.chrusciel/teaching/Energy/Energy.pdf.

[9] P.T. Chruściel, On the relation between the Einstein and the Komar expressions for the energy of the gravitational field, Ann. Inst. Henri Poincaré 42 (1985), 267-282. MR 797276 (86k:83018)

[10] _ The Hamiltonian mass and asymptotically anti-de Sitter spacetimes, Proceedings of the Symposium "100 Years Werner HeisenbergWorks and Impact" (Bamberg, 2001), vol. 50, 2002, pp. 624-629. MR MR1909102 (2003h:83034)

[11] P.T. Chruściel and M. Herzlich, The mass of asymptotically hyperbolic Riemannian manifolds, Pacific J. Math. 212 (2003), 231-264, arXiv:dgga/0110035. MR MR2038048 (2005d:53052)

[12] P.T. Chruściel, J. Jezierski, and J. Kijowski, The Hamiltonian mass of asymptotically Schwarzschild-de Sitter space-times, Phys. Rev. D87 (2013), 124015 (11 pp.), arXiv:1305.1014 [gr-qc].

[13] P.T. Chruściel, J. Jezierski, and S. Łęski, The Trautman-Bondi mass of hyperboloidal initial data sets, Adv. Theor. Math. Phys. 8 (2004), 83-139, arXiv:gr-qc/0307109. MR MR2086675 (2005j:83027)

[14] P.T. Chruściel, M.A.H. MacCallum, and D. Singleton, Gravitational waves in general relativity. XIV: Bondi expansions and the "polyhomogeneity" of Scri, Philos. Trans. Roy. Soc. London Ser. A 350 (1995), 113-141, arXiv:grqc/9305021. MR MR1325206 (97f:83025)

[15] P.T. Chruściel and G. Nagy, The Hamiltonian mass of asymptotically antide Sitter space-times, Class. Quantum Grav. 18 (2001), L61-L68, hepth/0011270.

[16] - The mass of spacelike hypersurfaces in asymptotically anti-de Sitter space-times, Adv. Theor. Math. Phys. 5 (2002), 697-754, arXiv:grqc/0110014.

[17] P.T. Chruściel and T.-T. Paetz, The mass of light-cones, Class. Quantum Grav. 31 (2014), 102001, arXiv1401.3789 [gr-qc].

[18] _ Light-cone initial data and smoothness of Scri. I. Formalism and results, Ann. H. Poincaré 16 (2015), 2131-2162, arXiv:1403.3558 [gr-qc]. MR 3383324

[19] P.T. Chruściel and W. Simon, Towards the classification of static vacuum space-times with negative cosmological constant, Jour. Math. Phys. 42 (2001), 1779-1817, arXiv:gr-qc/0004032. MR 1820431 (2002j:83013)

[20] C. Fefferman and C.R. Graham, Conformal invariants, Élie Cartan et les mathématiques d'aujourd'hui, The mathematical heritage of Élie Cartan, Sémin. Lyon 1984, Astérisque, No.Hors Sér. 1985, 95-116. 
[21] H. Friedrich, On the hyperbolicity of Einstein's and other gauge field equations, Commun. Math. Phys. 100 (1985), 525-543. MR MR806251 (86m:83009)

[22] _ On the existence of n-geodesically complete or future complete solutions of Einstein's field equations with smooth asymptotic structure, Commun. Math. Phys. 107 (1986), 587-609.

[23] - On the global existence and the asymptotic behavior of solutions to the Einstein-Maxwell-Yang-Mills equations, Jour. Diff. Geom. 34 (1991), 275-345. MR MR1131434 (92i:58191)

[24] _ Smooth non-zero rest-mass evolution across time-like infinity, Ann. Henri Poincaré 16 (2015), 2215-2238, arXiv:1311.0700 [gr-qc].

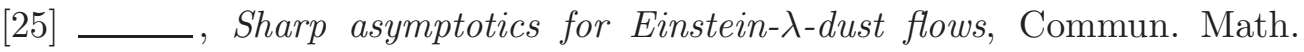
Phys. (2016), in press, arXiv:1601.04506 [gr-qc].

[26] C.R. Graham, Volume and area renormalizations for conformally compact Einstein metrics, The Proceedings of the 19th Winter School "Geometry and Physics" (Srní, 1999), no. 63, 2000, arXiv:math/9909042 [math.DG], pp. 31-42. MR 1758076 (2002c:53073)

[27] G.T. Horowitz and R.C. Myers, The AdS/CFT correspondence and a new positive energy conjecture for general relativity, Phys. Rev. D59 (1999), 026005 (12 pp.), arXiv:hep-th/9808079.

[28] T.-T. Paetz, Characteristic initial data and smoothness of Scri. II. Asymptotic expansions and construction of conformally smooth data sets, Jour. Math. Phys. 55 (2014), 102503, arXiv:1403.3560 [gr-qc].

[29] _ On characteristic Cauchy problems in general relativity, Ph.D. thesis, University of Vienna, 2014, http://homepage univie.ac .at/piotr. chrusciel/papers/Tim.pdf.

[30] Conformally covariant systems of wave equations and their equivalence to Einstein's field equations, Ann. H. Poincaré 16 (2015), 2059-2129, arXiv:1306.6204 [gr-qc]. MR 3383323

[31] R.K. Sachs, Gravitational waves in general relativity VIII. Waves in asymptotically flat space-time, Proc. Roy. Soc. London A 270 (1962), 103126. MR MR0149908 (26 \#7393)

[32] L.B. Szabados and P. Tod, A positive Bondi-type mass in asymptotically de Sitter spacetimes, Class. Quant. Grav. 32 (2015), 205011, arXiv:1505.06637 [gr-qc].

[33] J. Tafel, On the energy of a null cone, Class. Quantum Grav. 31 (2014), 235011, [Class. Quant. Grav.31,235011(2014)].

[34] L.A. Tamburino and J.H. Winicour, Gravitational fields in finite and conformal Bondi frames, Phys. Rev. 150 (1966), 1039-1053. 
[35] A. Trautman, Radiation and boundary conditions in the theory of gravitation, Bull. Acad. Pol. Sci., Série sci. math., astr. et phys. VI (1958), 407-412.

[36] X. Wang, Mass for asymptotically hyperbolic manifolds, Jour. Diff. Geom. 57 (2001), 273-299. MR MR1879228 (2003c:53044)

[37] E. Woolgar, The rigid Horowitz-Myers conjecture, (2016), arXiv:1602.06197. 